\title{
A CONTRIBUTION TO THE SNOWBED LIVERWORT FLORA OF THE TATRA MOUNTAINS (WESTERN CARPATHIANS, POLAND AND SLOVAKIA)
}

\author{
PIOTR GóRSKI \\ P. Górski, Department of Botany, Poznań University of Life Sciences, Wojska Polskiego 71 C, 60-625 Poznań, \\ Poland, e-mail: peter@up.poznan.pl
}

(Received: April 22, 2015. Accepted: July 20, 2015)

\begin{abstract}
AвstRact. The article is a first update of the list of liverwort localities published in A synopsis of liverworts occurring in the Tatra Mountains (Western Carpathians, Poland and Slovakia): checklist, distribution, and new data (GóRSKI \& VÁŇA 2014). It presents localities of 19 liverwort species collected during 2003-2014 in snowbeds in the entire area of the Tatra Mts. Moreover, for some species, localities from habitats other than snowbeds were given. Localities of the following liverworts are presented: Anthelia juratzkana, Cephalozia ambigua, C. bicuspidata, Diplophyllum taxifolium, Fuscocephaloziopsis albescens [= Pleurocladula albescens], Gymnocolea inflata, Gymnomitrion adustum [= Marsupella adusta], G. concinnatum, G. brevissimum [= Marsupella brevissima], Lophozia wenzelii, Marsupella boeckii, M. condensata, M. emarginata, M. sprucei, Moerckia blyttii, Nardia scalaris, Pseudolophozia sudetica [= Lophozia sudetica], Schistochilopsis opacifolia [= Lophozia incisa subsp. opacifolia] and Trilophozia quinquedentata [= Tritomaria quinquedentata].
\end{abstract}

KeY words: liverworts, snowbeds, Tatra Mts, Western Carpathians, Poland, Slovakia

\section{LIST OF LOCALITIES}

Explanations: 34 UDV2745 - grid square $(1 \mathrm{~km} \times$ $1 \mathrm{~km}$ ) adopted from the UTM/MGRS carthographic system; leg. PG - collected by P. Górski; liverwort nomenclature - GóRSKI \& VÁŇA (2014).

Anthelia juratzkana (Limpr.) Trevis.

Slovakia, High Tatra Mountains: 34UDV2745: Važecká dolina valley, near SE shore of Krivánske Zelené pleso lake, blocks of rock, $49.15792^{\circ} \mathrm{N}$, $20.00877^{\circ} \mathrm{E}, 49.15758^{\circ} \mathrm{N}, 20.0066^{\circ} \mathrm{E}$, alt. $2000 \mathrm{~m}$, leg. PG, 11.08.2010 (POZNB 21/2010, 177); Važecká dolina valley, blocks of rock $\mathrm{N}$ from $\mathrm{N}$ shore of Krivánske Zelené pleso lake, $49.16192^{\circ} \mathrm{N}, 20.00682^{\circ} \mathrm{E}$, alt. $2020 \mathrm{~m}$, leg. PG, 11.08.2010 (POZNB 593); 34UDV2746: Nefcerka valley, near W shore of Nižné Terianske pleso lake, alt. 1940 m, leg. PG, 2.08.2011 (POZNB 640); 34UDV2750: upper part of Zadna Tichá dolina valley, alt. 1770 m, leg. PG, 29.07.2009 (POZNB 25/2009); 34UDV2845: Furkotská dolina valley, above $\mathrm{N}$ shore of Nižné Wahlenbergovo pleso lake, $\quad 49.16032^{\circ} \mathrm{N}, \quad 20.02475^{\circ} \mathrm{E}, \quad 49.16042^{\circ} \mathrm{N}$, $20.02452^{\circ} \mathrm{E}, 49.16012^{\circ} \mathrm{N}, 20.02527^{\circ} \mathrm{E}$, alt. $2060 \mathrm{~m}$, $2065 \mathrm{~m}$, leg. PG, 12.08.2010 (POZNB 22/2010, 23/2010, 205); 34UDV2846: Nefcerka valley, rock boulders E from E shore of Nižné Terianske pleso lake, $49.16727^{\circ} \mathrm{N}, 20.01613^{\circ} \mathrm{E}$, alt. $2035 \mathrm{~m}$, leg. PG, 2.08.2012 (POZNB 1326); Nefcerka valley, the highest glacial cirque, below Furkotské sedlo pass, $49.16905^{\circ} \mathrm{N}, 20.02283^{\circ} \mathrm{E}$, alt. $2150 \mathrm{~m}$, leg. PG, 3.08.2011 (POZNB 1983); 34UDV2945: Mlynická dolina valley, below rocky walls descending from $\mathrm{Mt}$ Malé Solisko, alt. 2030 m, leg. PG, 15.08.2008 (POZNB 34/2008); Mlynická dolina valley, blocks of rock below Mt Malé Solisko, alt. 2015 m, leg. PG, 15.08.2008 (POZNB 105); 34UDV2946: Furkotská dolina valley, blocks of rock near SE shore of Vyšné Wahlenbergovo pleso lake, alt. $2100 \mathrm{~m}, 2125 \mathrm{~m}$, 2130 m, leg. PG, 8.08.2008 (POZNB 86, 90, 14/2008, 15/2008, 16/2008); Mlynická dolina valley, blocks of rock below rocky walls descending from Mt Furkotský štit, above Okrúhle pleso lake, alt. 2135 m, leg. PG, 10.08.2008 (POZNB 19/2008); Mlynická dolina valley, N slope of Bystrá lavká pass, rocky crevices, $49.1671^{\circ} \mathrm{N}, 20.02937^{\circ} \mathrm{E}$, alt. $2230 \mathrm{~m}, 2285 \mathrm{~m}, 2250$ 
$\mathrm{m}$, leg. PG, 10.08.2008, 12.08.2011 (POZNB 663, 17/2008, 18/2008); 34UDV2949: Temnosmrečinská dolina valley, rocky outcrops between Nižné Temnosmrečinské pleso and Vyšné Temnosmrečinské pleso lakes, alt. $1700 \mathrm{~m}$, leg. PG, 30.07.2009 (POZNB 31/2009); 34UDV3045: Mlynická dolina valley, near Volie pliesko lake, alt. $1915 \mathrm{~m}$, leg. PG, 11.08.2008 (POZNB 27/2008); 34UDV3046: Mlynická dolina valley, blocks of rock above $\mathrm{S}$ shore of Vyšné Kozie pleso lake, alt. 2110 m, leg. PG, 11.08.2008 (POZNB 22/2008); upper, NE part of Mlynická dolina valley, blocks of rock, below rocky walls descending from Mt Velka Capia veža, alt. 2100 m, leg. PG, 11.08.2008 (POZNB 24/2008, 25/2008); 34UDV3047: Hlinská dolina valley, bottom plateau below Výsné Kôprovské sedlo pass, alt. 1900 m, 1950 $\mathrm{m}$, leg. PG, 21.08.2006 (POZNB 93, 41/2006, 42/2006, 43/2006); 34UDV3048: Piargová dolinka valley, glacial cirque below Mt Čubrina, blocks of rock, alt. $1865 \mathrm{~m}, 1875 \mathrm{~m}$, leg. PG, 28.07.2009 (POZNB 44/2006, 18/2009, 20/2009, 22/2009); Temnosmrečinská dolina valley, on the sub-glacially eroded step of Piargová dolinka valley, $49.18793^{\circ} \mathrm{N}$, $20.04403^{\circ} \mathrm{E}$, alt. $1840 \mathrm{~m}$, leg. PG, 5.08.2011 (POZNB 658); 34UDV3148: Hincova dolina valley, rocky outcrops near N shore of Velké Hincovo pleso lake, alt. 1940 m, leg. PG, 12.08.2008 (POZNB 29/2008); 34UDV3247: Žabia Mengusovská dolina valley, Kotlina Žabich plies basin, blocks of rock $\mathrm{N}$ and above Velké Žabie Mengusovské pleso lake, alt. $1870 \mathrm{~m}$, $1935 \mathrm{~m}$, leg. PG, 13.08.2008 (POZNB 31/2008, 32/2008); 34UDV3344: Mengusovská dolina valley, near route from Popradské pleso lake to Mt Ostrva, alt. 1675-1885 m, leg. PG, 10.08.2010 (POZNB 211); 34UDV3346: Drača dolinka valley, above Drače pleso lake, blocks of rock, $49.16768^{\circ} \mathrm{N}, 20.089^{\circ} \mathrm{E}$, $49.16503^{\circ} \mathrm{N}, 20.08898^{\circ} \mathrm{E}$, alt. $2050 \mathrm{~m}$, leg. $\mathrm{PG}$, 5.08.2010 (POZNB 193, 196); 34UDV3347: Žabia Mengusovská dolina valley, Kotlinka pod Vahou basin, near hut, alt. $2150 \mathrm{~m}$, leg. PG, 13.08.2008 (POZNB 91); 34UDV3349: Žabia Bielovodská dolina valley, blocks of rock above $S$ shore of Vyšné Žabie Bielovodské pleso lake, alt. $1700 \mathrm{~m}, 1730 \mathrm{~m}$, leg. PG, 15.08.2009 (POZNB 56, 37/2009, 39/2009); 34UDV3444: upper part of Štôlska dolina valley, below Lúčne sedlo pass, $49.15305^{\circ} \mathrm{N}, 20.10683^{\circ} \mathrm{E}$, alt. 2000 m, 2045 m, leg. PG, 10.08.2010 (POZNB 178, 20/2010); 34UDV3445: Zlomisková dolina valley, Zlomisková roveň, $49.16037^{\circ} \mathrm{N}, 20.09912^{\circ} \mathrm{E}$, alt. 1825 m, leg. PG, 9.08.2011 (POZNB 671); Zlomisková dolina valley, Kotlina Ladového plesa basin, near SE shore of Ladové pleso lake, $49.16285^{\circ} \mathrm{N}$, 20.10755 ${ }^{\circ}$, alt. $1930 \mathrm{~m}$, leg. PG, 6.08.2010 (POZNB 19/2010); Zlomisková dolina valley, Kotlina Ladového plesa basin, rocky outcrops above S shore of Ladové pleso lake, below Lúčne sedlo pass, $49.16193^{\circ} \mathrm{N}, 20.10615^{\circ} \mathrm{E}, 49.16208^{\circ} \mathrm{N}, 20.10652^{\circ} \mathrm{E}$, alt. 1975 m, 1980 m, 2005 m, leg. PG, 5.08.2010
(POZNB 228, 15/2010, 16/2010); 34UDV3446: Rumanova dolinka valley, lower part of the valley, snowbed with Gnaphalium supinum, $49.17002^{\circ} \mathrm{N}$, 20.10235 ${ }^{\circ}$, alt. $2165 \mathrm{~m}$, leg. PG, 3.08.2010 (POZNB 218); Rumanova dolinka valley, near $\mathrm{E}$ shore of Vyšné Rumanovo pliesko lake, $49.1719^{\circ} \mathrm{N}, 20.1008^{\circ} \mathrm{E}$, alt. 2125 m, leg. PG, 3.08.2010 (POZNB 9/2010); Rumanova dolinka valley, near $\mathrm{N}$ shore of Vyšné Rumanovo pliesko lake, $49.17202^{\circ} \mathrm{N}, 20.1006^{\circ} \mathrm{E}$, $49.17202^{\circ} \mathrm{N}, 20.1006^{\circ} \mathrm{E}, 49.17202^{\circ} \mathrm{N}, 20.1006^{\circ} \mathrm{E}$, alt. $2125 \mathrm{~m}$, leg. PG, 4.08.2010 (POZNB 11/2010, 12/2010, 13/2010); Rumanova dolinka valley, near NE shore of Vyšné Rumanovo pliesko lake, $49.17202^{\circ} \mathrm{N}, 20.10063^{\circ} \mathrm{E}$, alt. $2125 \mathrm{~m}$, leg. PG, 4.08.2010 (POZNB 10/2010); Rumanova dolinka valley, near NE shore of Rumanovo pleso lake, $49.16842^{\circ} \mathrm{N}, 20.0997^{\circ} \mathrm{E}$, alt. $2090 \mathrm{~m}$, leg. PG, 3.08.2010 (POZNB 226); Zlomisková dolina valley, Kotlina Ladového plesa basin, near W shore of Ladové pleso lake, $49.16352^{\circ} \mathrm{N}, 20.1049^{\circ} \mathrm{E}$, alt. 1930 m, leg. PG, 6.08.2010 (POZNB 225); 34UDV3447: Ťažká dolina valley, upper glacial cirque, blocks of rock below Mt Ganek and Mt Vysoká, alt. 1990 m, leg. PG, 20.08.2009 (POZNB 57/2009); Ťažká dolina valley, upper glacial cirque, blocks of rock below $\mathrm{Mt}$ Kačacia veža, alt. 1820 m, leg. PG, 24.08.2009 (POZNB 62/2009); Ťažká dolina valley, upper glacial cirque, near $\mathrm{E}$ shore of Zmarzlé pleso lake, below rocky walls descending from Mt Prédná Pustá veža, alt. 1765 m, leg. PG, 20.08.2009 (POZNB 59/2009); 34UDV3448: Spádová dolinka valley, SW part of the glacial cirque, blocks of rock below rocky walls descending from Mt Malé Rysy, alt. 1995 m, leg. PG, 25.08.2009 (POZNB 66/2009); Ťažká dolina valley, upper glacial cirque, near $\mathrm{N}$ shore of Zmarzlé pleso lake, below rocky walls descending from Mt Prédná Pustá veža, alt. 1760 m, leg. PG, 20.08.2009 (POZNB 87); upper part of Žabia Bielovodská dolina valley, below rocky walls between Mlynárovo sedlo and Mt Velký Žabi štít, alt. 1885 m, 1890 m, 1920 m, leg. PG, 17.08.2009, 19.08.2009 (POZNB 46/2009, 47/2009, 50/2009, 51/2009, 52/2009); 34UDV3449: Žabia Bielovodská dolina valley, blocks of rock near SE shore of Nižné Žabie Bielovodské pleso lake, alt. 1675 m, leg. PG, 14.08.2009 (POZNB 34/2009); Žabia Bielovodská dolina valley, E from $\mathrm{S}$ shore of Nižné Žabie Bielovodské pleso lake, below rocky walls descending from Mt Prostredný Mlynár, alt. $1735 \mathrm{~m}$, leg. PG, 16.08.2009 (POZNB 40/2009, 41/2009); Žabia Bielovodská dolina valley, NW from Mlynárovo sedlo pass, alt. 1800 m, 1920 m, leg. PG, 15.08.2009 (POZNB 36/2009, 44/2009, 45/2009); 34UDV3544: lower part of Štôlska dolina valley, above Tatranská Magistrála route, blocks of rock, $49.14893^{\circ} \mathrm{N}$, $20.11088^{\circ} \mathrm{E}, 49.14893^{\circ} \mathrm{N}, 20.11088^{\circ} \mathrm{E}$, alt. $1935 \mathrm{~m}$, leg. PG, 10.08.2010 (POZNB 176, 202); 34UDV3545: Batizovská dolina valley, blocks of rock near S shore of Pliesko pod Kostolikom lake, $49.1603^{\circ} \mathrm{N}$, 
$20.12135^{\circ} \mathrm{E}$, alt. $2045 \mathrm{~m}$, leg. PG, 1.08.2010 (POZNB 2/2010); Batizovská dolina valley, blocks of rock near SE shore of Pliesko pod Kostolikom lake, $49.16117^{\circ} \mathrm{N}$, 20.12187E, alt. $2055 \mathrm{~m}$, leg. PG, 1.08.2010 (POZNB 1/2010); 34UDV3546: Kačacia dolina valley, upper glacial cirque (Żelazny Ogród), blocks of rock below Východná Železná brána pass, alt. 1980 m, 1985 m, 2000 m, 2015 m, leg. PG, 16.09.2009 (POZNB 143, 144, 73/2009, 74/2009, 75/2009); Zlomisková dolina valley, Železná kotlina basin (below Východná Železná braná pass), $49.16393^{\circ} \mathrm{N}, 20.11097^{\circ} \mathrm{E}$, alt. $2080 \mathrm{~m}$, leg. PG, 6.08.2010 (POZNB 214); 34UDV3547: Kačacia dolina valley, gully descending from Mt Zlobivá, alt. 1700 m, leg. PG, 22.08.2007 (POZNB 6/2007); Kačacia dolina valley, Kačací žlab gully, alt. 1825 m, leg. PG, 22.08.2007 (POZNB 36); Kačacia dolina valley, lower glacial cirque, alt. 1645 $\mathrm{m}$, leg. PG, 22.08.2007 (POZNB 7/2007); 34UDV3548: Ťažká dolina valley, upper part of the lower glacial cirque, blocks of rock below Mt Pustá stráž, alt. 1820 m, leg. PG, 26.08.2009 (POZNB 68); 34UDV3645: Batizovská dolina valley, $\mathrm{N}$ slope of $\mathrm{Mt}$ Kostolík, Polytrichetum norvegici, $49.16162^{\circ} \mathrm{N}$, $20.12412^{\circ} \mathrm{E}$, alt. $2240 \mathrm{~m}$, leg. PG, 2.08.2010 (POZNB 184); Batizovská dolina valley, Vyšna Batizovská roveň, Batizovské oká lakes, $49.1631^{\circ} \mathrm{N}, 20.12667^{\circ} \mathrm{E}$, alt. $2250 \mathrm{~m}$, leg. PG, 2.08.2010 (POZNB 180); 34UDV3646: Kačacia dolina valley, upper glacial cirque (Gierlachowski Ogród), below rocky walls descending from Mt Zadný Gerlachovský štít, alt. 1975 m, leg. PG, 16.09.2009 (POZNB 133); 34UDV3647: Kačacia dolina valley, on the sub-glacially eroded step of Litvorová dolina valley, alt. $1685 \mathrm{~m}$, leg. PG, 15.09.2009 (POZNB 141); Litvorová dolina valley, below rocky walls descending from Mt Litvorový štít, alt. 1835 m, leg. PG, 15.09.2009 (POZNB 72/2009); Litvorová dolina valley, near S shore of Litvorové pleso lake, $49.17643^{\circ} \mathrm{N}, 20.12872^{\circ} \mathrm{E}$, alt. $1860 \mathrm{~m}$, leg. PG, 16.09.2009 (POZNB 142); Litvorová dolina valley, rocky outcrops $\mathrm{E}$ from Litvorové pleso lake, alt. $1880 \mathrm{~m}, 1940 \mathrm{~m}, 1990 \mathrm{~m}$, leg. PG, 17.08.2006 (POZNB 34/2006, 35/2006, 36/2006); 34UDV3746: Velická dolina valley, Velická kotlina basin, blocks of rock near Vyšné Velické plieska lakes, $49.1723^{\circ} \mathrm{N}$, 20.13588 ${ }^{\circ}$, alt. $2125 \mathrm{~m}$, leg. PG, 8.08.2012 (POZNB 1145); Velická dolina valley, Vyšná Kvetnica, alt. 1885 m, leg. PG, 1.08.2006 (POZNB 113, 23/2006); 34UDV3747: Svištová (Bielovodská) dolina valley, Zmarznutý kotol basin, alt. 2005 m, 2035 m, 2065 m, leg. PG, 1.08.2006 (POZNB 24/2006, 25/2006, 26/2006); Svištová (Bielovodská) dolina valley, Zmarznutý kotol basin, near Zmarznuté pleso lake, $49.17645^{\circ} \mathrm{N}, 20.13918^{\circ} \mathrm{E}$, alt. $2040 \mathrm{~m}$, leg. PG, 26.09.2011 (POZNB 482); Velická dolina valley, blocks of rock below Mt Velický štít, alt. 2080 m, leg. PG, 1.08.2006 (POZNB 58); Zmarznutý kotol basin, rocky walls below Polský hrebeň pass, $49.17487^{\circ} \mathrm{N}$, $20.13838^{\circ} \mathrm{E}$, alt. $2060 \mathrm{~m}$, leg. PG, 9.08.2012 (POZNB
1155); Veĺka Studená dolina valley, Kotlina pod Prielomom basin, alt. $2035 \mathrm{~m}$, leg. PG, 24.08.2007 (POZNB 16/2007); 34UDV3848: Rovienky valley, Zadné Rovienky, $\quad 49.18747^{\circ} \mathrm{N}, \quad 20.15157^{\circ} \mathrm{E}$, $49.18767^{\circ} \mathrm{N}, 20.15355^{\circ} \mathrm{E}, 49.18745^{\circ} \mathrm{N}, 20.15342^{\circ} \mathrm{E}$, alt. $1925 \mathrm{~m}, 1930 \mathrm{~m}, 1950 \mathrm{~m}$, leg. PG, 25.09.2011 (POZNB 497, 500, 604); Rovienky, Zadné Rovienky, above Rovienkový vodopad waterfall, blocks of rock, $49.18848^{\circ} \mathrm{N}, 20.1513^{\circ} \mathrm{E}$, alt. $1875 \mathrm{~m}$, leg. PG, 25.09.2011 (POZNB 494); 34UDV3850: lower part of Suchá (Javorová) dolina valley, alt. $1625 \mathrm{~m}$, leg. PG, 18.08.2006 (POZNB 112); 34UDV3946: Veĺka Studená dolina valley, Dolinka nad Vareškovým Plesom valley, alt. $1845 \mathrm{~m}, 1850 \mathrm{~m}, 1870 \mathrm{~m}$, leg. PG, 23.08.2007 (POZNB 51, 9/2007, 10/2007, 13/2007); 34UDV3947: Veĺka Studená dolina valley, rocky outcrops E from Dlhé pleso lake, above stream, alt. 1875 m, leg. PG, 23.08.2007 (POZNB 8/2007); Veĺka Studená dolina valley, rocky walls near stream between Dlhé pleso and Vareškové pleso lake, alt. $1815 \mathrm{~m}$, leg. PG, 23.08.2007 (POZNB 14/2007); 34UDV3948: Zadná Javorová dolina valley, glacial cirque below Mt Ostrý štít, alt. 2210 m, 2125 m, leg. PG, 16.08.2006 (POZNB 28/2006, 31/2006); 34UDV3949: Zadná Javorová dolina valley, Sobkova úboč, blocks of rock, alt. $1985 \mathrm{~m}$, leg. PG, 21.08.2007 (POZNB 94); 34UDV3950: Suchá (Javorová) dolina valley, rocky walls with northern exposure, alt. $1930 \mathrm{~m}$, leg. PG, 18.08.2006 (POZNB 66); upper part of Suchá (Javorová) dolina valley, alt. $1860 \mathrm{~m}$, leg. PG, 18.08.2006 (POZNB 38/2006); 34UDV4048: Veĺka Studená dolina valley, Strelecké polia, $49.18543^{\circ} \mathrm{N}, 20.17883^{\circ} \mathrm{E}$, $49.18443^{\circ} \mathrm{N}, 20.18188^{\circ} \mathrm{E}$, alt. $2020 \mathrm{~m}, 2105 \mathrm{~m}$, leg. PG, 11.08.2011 (POZNB 659, 669); 34UDV4049: Zadná Javorová dolina valley, below rocky walls descending from Mt Ostrí štít, alt. 2130 m, 2140 m, leg. PG, 16.08.2006 (POZNB 38, 75); 34UDV4050: Čierna Javorová dolina valley, Čierna záhrada, base of rocky walls at the mouth of Ladova dolinka valley, $49.20685^{\circ} \mathrm{N}, 20.17762^{\circ} \mathrm{E}$, alt. $1670 \mathrm{~m}$, leg. PG, 25.08.2010 (POZNB 166, 24/2010); Čierna Javorová dolina valley, upper glacial cirque (Čierne mlaki), $49.2084^{\circ} \mathrm{N}, 20.18747^{\circ} \mathrm{E}$, alt. $1940 \mathrm{~m}$, leg. PG, 26.08.2010 (POZNB 163); 34UDV4051: Čierna Javorová dolina valley, Čierne mlaki, on rocks dripping with water, $49.20945^{\circ} \mathrm{N}, 20.1887^{\circ} \mathrm{E}$, alt. $1910 \mathrm{~m}$, leg. PG, 26.08.2010 (POZNB 171); 34UDV4148: Kotlina Piatich Spišských Plies valley, near Nižne Spišské pleso lake, alt. 1990 m, leg. PG, 29.08.2007 (POZNB 28/2007); Malá Studená dolina valley, Dolina pod Sedielkom valley, alt. 2065 m, leg. PG, 21.08.2007 (POZNB 2/2007); upper part of Malá Studená dolina valley, alt. $1935 \mathrm{~m}, 1945 \mathrm{~m}$, leg. PG, 29.08.2007 (POZNB 29/2007, 30/2007); 34UDV4149: Kotlina Piatich Spišských Plies valley, above W shore of Prostredné Spišské pleso lake, alt. 2000 m, 2005 m, leg. PG, 27.08.2007 (POZNB 18/2007, 19/2007, 20/2007, 21/20070); Kotlina Piatich Spišských Plies 
valley, blocks of rock E from Prostredné Spišské pleso lake, alt. 2035 m, leg. PG, 29.08.2007 (POZNB 25/2007); Kotlina Piatich Spišských Plies valley, blocks of rock NW from Velké Spišské pleso lake, alt. 2070 m, leg. PG, 27.08.2007 (POZNB 24/2007); Kotlina Piatich Spišských Plies valley, near NE shore of Vyšne Spišské pleso lake, $49.19468^{\circ} \mathrm{N}, 20.19688^{\circ} \mathrm{E}$, alt. 2024 m, leg. PG, 22.09.2010 (POZNB 236); Kotlina Piatich Spišských Plies valley, near W shore of Prostredné Spišské pleso lake, alt. 2010 m, leg. PG, 29.08.2007 (POZNB 27/2007); 34UDV4150: Čierna Javorová dolina valley, Czarny Bandzioch, $49.20667^{\circ} \mathrm{N}, 20.19423^{\circ} \mathrm{E}, 49.20782^{\circ} \mathrm{N}, 20.19433^{\circ} \mathrm{E}$, $49.20817^{\circ} \mathrm{N}, 20.19247^{\circ} \mathrm{E}$, alt. $2010 \mathrm{~m}, 2050 \mathrm{~m}, 2075$ $\mathrm{m}$, leg. PG, 26.08.2010 (POZNB 172, 27/2010, 28/2010); Čierna Javorová dolina valley, Czarny Bandzioch, near Čierny potok stream, $49.20842^{\circ} \mathrm{N}$, 20.19167 E, alt. 1995 m, leg. PG, 26.08.2010 (POZNB 29/2010); 34UDV4151: Kolová dolina valley, Kotlinka pod Kolovym sedlom basin, $49.21647^{\circ} \mathrm{N}$, 20.20273 E, alt. $2000 \mathrm{~m}$, leg. PG, 1.09.2011 (POZNB 646); Kolová dolina valley, upper part of Kotlinka pod Kolovym sedlom basin, below Kolové sedlo pass, $49.21433^{\circ} \mathrm{N}, 20.20125^{\circ} \mathrm{E}$, alt. $2095 \mathrm{~m}$, leg. PG, 1.09.2011 (POZNB 467); 34UDV4152: Kolová dolina valley, blocks of rock in upper part of the valley, $49.21825^{\circ} \mathrm{N}, 20.20028^{\circ} \mathrm{E}$, alt. $1820 \mathrm{~m}$, leg. $\mathrm{PG}$, 1.09.2011 (POZNB 449, 451); 34UDV4250: upper part of Velká Zmrzlá dolina valley, Barania kotlina basin, $49.20268^{\circ} \mathrm{N}, 20.20448^{\circ} \mathrm{E}$, alt. $2085 \mathrm{~m}$, leg. PG, 27.09.2011 (POZNB 542); Velká Zmrzlá dolina valley, blocks of rock above the sub-glacially eroded step of the valley, $49.20442^{\circ} \mathrm{N}, 20.20898^{\circ} \mathrm{E}, 49.20437^{\circ} \mathrm{N}$, $20.20968^{\circ} \mathrm{E}$, alt. $1900 \mathrm{~m}, 1940 \mathrm{~m}$, leg. PG, 27.09.2011 (POZNB 545, 557); 34UDV4251: Červena dolina valley, near Červené pleso lake, alt. $1815 \mathrm{~m}$, leg. PG, 25.09.2007 (POZNB 32/2007, 33/2007); 34UDV4350: Dolina Kežmarskej Bielej Vody valley, near $\mathrm{S}$ shore of Zelené pleso lake, alt. $1541 \mathrm{~m}$, leg. PG, 21.08.2009 (POZNB 71); 34UDV4449: E slope of Mt Huncowský štít, above route from Skalnaté pleso lake to Mt Veĺká Svištovka, alt. 1895 m, leg. PG, 17.07.2011 (POZNB 631); 34UDV4450: N slope of Mt Veĺká Svištovka, rocky crevices, alt. 1975 m, leg. PG, 30.09.2006 (POZNB 21).

Slovakia, Western Tatra Mountains: 34UDV0452: Mt Salatín, snowbed on the top, alt. 2015 m, leg. PG, 19.07.2008 (POZNB 273); 34UDV0453: Roháčska dolina valley, glacial cirque below Mt Brestová (from the north side), alt. $1700 \mathrm{~m}, 1780 \mathrm{~m}$, leg. PG, 23.07.2006 (POZNB 76, 17/2006); 34UDV0550: Dolina Parichvost valley, on route below Baníkovské sedlo pass, alt. $1875 \mathrm{~m}$, leg. PG, 16.07.2008 (POZNB 37); Spálená dolina valley, below Baníkovské sedlo pass, alt. $1855 \mathrm{~m}$, leg. PG, 19.07.2006 (POZNB 23); 34UDV0551: Jalovecká dolina valley, E part of Hlboká dolina valley (Vrece), below rocky walls descending from Mt Pachol'a, alt. 1740 m, 1815 m, 1895 m, leg. PG, 19.07.2008, 20.07.2008 (POZNB 22, 95, 4/2008); 34UDV0650: Spálená dolina valley, below rocky walls descending from Mt Baníkov, alt. $1885 \mathrm{~m}$, leg. PG, 19.07.2006 (POZNB 3/2006); 34UDV0750: upper part of Smutná dolina valley, below Smutné sedlo pass, alt. $1825 \mathrm{~m}$, leg. PG, 18.07.2006 (POZNB 101); 34UDV0751: Roháčska dolina valley, blocks of rock near Horne Roháčske pleso lake, alt. 1730 m, 1755 m, leg. PG, 24.07.2006 (POZNB 21/2006, 22/2006); Spálená dolina valley, near route to Roháčske plesa lakes, alt. 1600 m, leg. PG, 24.07.2006 (POZNB 77); 34UDV0847: below Jamina pass (between Mt Baranec and Mt Smrek), upper part of Pusté gully, alt. $1815 \mathrm{~m}$, leg. PG, 21.07.2006 (POZNB 82); 34UDV0849: Žiarska dolina valley, Malé Závraty, below Žiarskie sedlo pass, in the cirque below Mt Plačlivý Roháč, alt. 1785 m, 1835 m, leg. PG, 20.07.2006 (POZNB 7/2006, 9/2006); snowbed on a ridge between Žiarskie sedlo pass and Mt Smrek, alt. 2025 m, leg. PG, 21.07.2006 (POZNB 13/2006, 14/2006); Jamnícka dolina valley, Roháčky kotol basin, on soil near Plačlivé pliesko lake, alt. $1785 \mathrm{~m}$, leg. PG, 20.07.2006 (POZNB 43); 34UDV0950: Smutná dolina valley, below rocky walls descending from Mt Ostrý Roháč, alt. 1810 m, leg. PG, 18.07.2006 (POZNB 83); Zadná Jamnícka dolina valley (Kocioł Jamnickich Stawów), near W shore of Jamnícke Pleso Vyšné lake, alt. 1830 m, leg. PG, 25.08.2008 (POZNB 96); 34UDV1250: Račkova dolina valley, Račkov Zadok, glacial cirque below Mt Jakubiná, alt. $1810 \mathrm{~m}$, $1830 \mathrm{~m}, 1845 \mathrm{~m}$, leg. PG, 10.08.2004, 21.07.2009 (POZNB 1/2009, 2/2009, 4/2004, 5/2004, 6/2004); Račkova dolina valley, Račkov Zadok, near W shore of Vyšné Račkove pleso lake, $49.19937^{\circ} \mathrm{N}, 19.8052^{\circ} \mathrm{E}$, alt. $1730 \mathrm{~m}$, leg. PG, 27.08.2011 (POZNB 455); Spálená dolina valley, below Baníkovské sedlo pass, rocky outcrops E from a route, alt. $1875 \mathrm{~m}$, leg. PG, 19.07.2006 (POZNB 2/2006); 34UDV1548: Bystrá dolina valley, near SE shore of Vel'ké Bystré pleso lake, $49.18258^{\circ} \mathrm{N}, 19.84308^{\circ} \mathrm{E}$, alt. $1875 \mathrm{~m}$, leg. $\mathrm{PG}$, 26.08.2011 (POZNB 617); 34UDV1648: Kobyla ridge (SE from Mt Bystrá), rocky outcrops near route to Bystrá dolina valley, $49.18118^{\circ} \mathrm{N}, 19.84977^{\circ} \mathrm{E}$, alt. $1990 \mathrm{~m}$, leg. PG, 26.08.2011 (POZNB 675); 34UDV1649: upper part of Kamenistá dolina valley, glacial cirque below Mt Blyšt, close to Pyšne sedlo pass, alt. $1875 \mathrm{~m}$, leg. PG, 29.08.2008 (POZNB 38/2008).

Poland, High Tatra Mountains: 34UDV2752: Dolina Gąsienicowa valley, glacial cirque below $\mathrm{Mt}$ Świnica, rocky outcrops S from Zadni Staw Gąsienicowy lake, alt. 1910 m, leg. PG, 20.08.2005 (POZNB 24/2005); Dolina Gąsienicowa valley, glacial cirque below Mt Świnica, rocky outcrops SE from Zadni Staw Gąsienicowy lake, alt. $1875 \mathrm{~m}$, leg. PG, 20.08.2005 (POZNB 25/2005); Dolina Gąsienicowa valley, S from Długi Staw Gąsienicowy lake, rocky walls dripping with water, $49.2255^{\circ} \mathrm{N}, 20.00795^{\circ} \mathrm{E}$, 
alt. $1800 \mathrm{~m}$, leg. PG, 20.09.2010 (POZNB 245); 34UDV2753: Dolina Gąsienicowa valley, near Kurtkowiec lake, $49.22845^{\circ} \mathrm{N}, 20.00313^{\circ} \mathrm{E}$, alt. $1685 \mathrm{~m}$, leg. PG, 30.06.2010 (POZNB 195); Dolina Gąsienicowa valley, near S shore of Długi Staw lake, Salicetum herbaceae, $49.22595^{\circ} \mathrm{N}, 20.00882^{\circ} \mathrm{E}$, alt. $1780 \mathrm{~m}$, leg. PG, 20.09.2010 (POZNB 276); 34UDV2851: W part of Dolina Pięciu Stawów Polskich valley, snowbeds below Gładka Przełęcz pass, alt. 1850 m, leg. PG, 27.07.2005 (POZNB 17/2005); Dolina Pięciu Stawów Polskich valley, sub-glacially eroded step of Dolinka Pusta valley, blocks of rock, $49.21427^{\circ} \mathrm{N}$, 20.0227 ${ }^{\circ}$ E, alt. $1960 \mathrm{~m}$, leg. PG, 24.08.2012 (POZNB 1964); 34UDV2852: Dolina Gąsienicowa valley, above and SW from Zmarzły Staw Gasienicowy, alt. 1800 m, leg. PG, 1.08.2003, 21.09.2010 (POZNB 101/2003, 237); Dolina Gąsienicowa valley, near SW shore of Zmarzły Staw Gąsienicowy lake, Salicetum herbaceae, $49.22377^{\circ} \mathrm{N}, 20.02213^{\circ} \mathrm{E}$, alt. $1778 \mathrm{~m}$, leg. PG, 21.09.2010 (POZNB 232); Dolina Gąsienicowa valley, $\mathrm{S}$ and above Zmarzly Staw Gąsienicowy lake, alt. $1800 \mathrm{~m}$, leg. PG, 1.08.2003, 2.08.2003 (POZNB 447, 98/2003, 99/2003, 112/2003); Dolina Gąsienicowa valley, above Zmarzły Staw Gąsienicowy lake, blocks of rock, alt. $1820 \mathrm{~m}, 1845 \mathrm{~m}$, leg. PG, 2.08.2003, 21.08.2005 (POZNB 137, 302, 29/2005); Dolina Gąsienicowa valley, at the mouth of Zawratowy Żleb gully, alt. 1950 m, 1955 m, leg. PG, 1.08.2003 (POZNB 105/2003, 106/2003); Dolina Gąsienicowa valley, below rocky walls descending from Zmarzła Przełęcz pass, alt. 2015 m, leg. PG, 4.08.2003 (POZNB 121/2003); Dolina Gąsienicowa valley, below rocky walls descending from Mt Zmarzłe Czuby, alt. 1930 m, leg. PG, 4.08.2003 (POZNB 117/2003); Dolina Gasienicowa valley, below Zawratowy Żleb gully, alt. 1825 m, 1840 m, leg. PG, 1.08.2003 (POZNB 103/2003, 104/2003, 107/2003); Dolina Gąsienicowa valley, Zadnie Koło, glacial cirque below Mylna Przełęcz pass, $49.22275^{\circ} \mathrm{N}, 20.01258^{\circ} \mathrm{E}$, alt. $1975 \mathrm{~m}$, leg. PG, 30.08.2011 (POZNB 680); 34UDV2853: Dolina Gąsienicowa valley, glacial cirque below Mt Świnica, blocks of rock near Długi Staw Gąsienicowy lake, alt. 1845 m, leg. PG, 20.08.2005 (POZNB 21/2005); Dolina Gąsienicowa valley, rocky rubble above Czarny Staw Gąsienicowy lake, below Mt Kościelec, alt. 1630 m, leg. PG, 31.07.2003 (POZNB 932); 34UDV2950: Dolina Pięciu Stawów Polskich valley, above Czarny Staw lake, below Kotelnica ridge, blocks of rock, alt. 1765 m, leg. PG, 27.07.2005 (POZNB 445); Dolina Pięciu Stawów Polskich valley, below Czarna Ławka pass, alt. $1870 \mathrm{~m}$, leg. PG, 27.07.2005 (POZNB 12/2005, 13/2005); Dolina Pięciu Stawów Polskich valley, below rocky walls descending from Mt Wyżni Liptowski Kostur, alt. 1940 m, 2005 m, 2040 m, leg. PG, 25.07.2005 (POZNB 1/2005, 3/2005, 2/2005); 34UDV2952: Dolinka Kozia valley, rocky rubble filling the valley bottom, alt. 1920 m, 1935 m, 1950 m, leg. PG, 20.08.2005, 21.08.2005 (POZNB 111/2003, $23 / 2005,27 / 2005$ ); Dolinka Kozia valley, rocky rubble filling the valley bottom, $49.222^{\circ} \mathrm{N}, 20.02657^{\circ} \mathrm{E}$, alt. 1960 m, leg. PG, 31.08.2011 (POZNB 468); upper part of Dolinka Kozia valley, below rocky walls descending from Orla Perć ridge, alt. 2050 m, 2055 m, leg. PG, 2.08.2003 (POZNB 108/2003, 109/2003); 34UDV2953: Dolina Pańszczycy valley, below rocky walls descending from Pańszczycka Przełęcz pass, alt. 1985 m, 1995 m, leg. PG, 3.08.2003 (POZNB 113/2003, 114/2003, 115/2003); 34UDV3049: Dolina za Mnichem valley, below rocky walls descending from Wrota Chałubińskiego pass, alt. $1850 \mathrm{~m}$, leg. PG, 16.08.2004 (POZNB 18/2004); Dolina za Mnichem valley, below Mt Cubryna, snowbed among blocks of rock, alt. 2000 m, leg. PG, 16.08.2004 (POZNB 21/2004, 22/2004); Dolina za Mnichem valley, below rocky walls descending from Mt Zadni Mnich, alt. 1980 m, leg. PG, 17.08.2004 (POZNB 23/2004); Dolina za Mnichem valley, near Wyżni Staw Staszica lake, alt. 1820 m, leg. PG, 16.08.2004 (POZNB 939); Dolina za Mnichem valley, rocky rubble filling the valley bottom, alt. $1790 \mathrm{~m}, 1820$ m, leg. PG, 16.08.2004, 27.09.2007 (POZNB 116, 15/2004, 37/2007); 34UDV3050: Dolina Pięciu Stawów Polskich valley, Czerwony Piarg scree, near route to Szpiglasowa Przełęcz pass, alt. 1955 m, leg. PG, 25.07.2005 (POZNB 4/2005); 34UDV3051: Dolina Pięciu Stawów Polskich valley, S from Przedni Staw Polski lake, alt. 1670 m, leg. PG, 25.07.2005 (POZNB 6/2005); Dolina Pięciu Stawów Polskich valley, snowbed near $S$ shore of Przedni Staw Polski lake, alt. 1665 m, leg. PG, 26.07.2005 (POZNB 568); 34UDV3151: Dolina Pięciu Stawów Polskich valley, below rocky walls descending from Marchwiczna Przełęcz pass, alt. 1810 m, 1815 m, $1900 \mathrm{~m}$, leg. PG, 26.07.2005 (POZNB 8/2005, 9/2005, 10/2005); 34UDV3153: upper part of Dolina Waksmundzka valley, on soil in snowbed, alt. $1950 \mathrm{~m}$, leg. PG, 22.08.2005 (POZNB 136, 566); 34UDV3248: below Mt Kazalnica, rocks near route to Mięguszowiecka Przełęcz pod Chłopkiem pass, $49.18645^{\circ} \mathrm{N}$, 20.06803 ${ }^{\circ}$ E, alt. $1975 \mathrm{~m}$, leg. PG, 2.09.2014 (POZNB 1912); Dolina Rybiego Potoku valley, rocky outcrops above Czarny Staw pod Rysami lake, alt. 1715 m, leg. PG, 18.08.2004 (POZNB 24/2004).

Poland, Western Tatra Mountains: 34UDV1150: Dolina Jarząbcza valley, below rocky walls descending from Niska Przełęcz pass, alt. 1730 m, leg. PG, 9.08.2004 (POZNB 3/2004); 34UDV1251: Dolina Jarząbcza valley, NW slope of Mt Kończysty Wierch (Jarząbczy Kopieniec), alt. 1700 m, 1710 m, leg. PG, 28.09.2003, 10.08.2004 (POZNB 148, 8/2004); 34UDV1450: Dolina Starorobociańska valley, Zadnie Zagony, alt. 1700 m, leg. PG, 11.08.2004 (POZNB 9/2004); 34UDV1649: Pyszniańska Przełęcz pass, $\mathrm{N}$ slope, alt. $1790 \mathrm{~m}$ (PG, 2004, observation); 34UDV1749: NW slope of Mt Kamienista, rocky 
crevices below the summit, $49.19628^{\circ} \mathrm{N}, 19.86753^{\circ} \mathrm{E}$, alt. $2045 \mathrm{~m}$, leg. PG, 4.08.2012 (POZNB 1147); 34UDV2055: upper part of Kobylarzowy Żleb pod Małołączniakiem gully, rocky crevices with northern exposure, $49.24387^{\circ} \mathrm{N}, 19.91082^{\circ} \mathrm{E}$, alt. $1700 \mathrm{~m}$, leg. PG, 22.11.2011 (POZNB 843); 34UDV2553: Mt Kasprowy Wierch, NW slope, below the summit, alt. 1970 m, leg. PG, 30.06.2010 (POZNB 220).

\section{Cephalozia ambigua C. Massal.}

Slovakia, High Tatra Mountains: 34UDV2846: Nefcerka valley, the highest glacial cirque, below Furkotské sedlo pass, $49.16905^{\circ} \mathrm{N}, 20.02283^{\circ} \mathrm{E}$, alt. $2150 \mathrm{~m}$, leg. PG, 3.08.2011 (POZNB 1983).

\section{Cephalozia bicuspidata (L.) Dumort.}

Slovakia, High Tatra Mountains: 34UDV2745: Važecká dolina valley, blocks of rock near SE shore of Krivánske Zelené pleso lake, $49.15758^{\circ} \mathrm{N}, 20.0066^{\circ} \mathrm{E}$, $49.15792^{\circ} \mathrm{N}, \quad 20.00877^{\circ} \mathrm{E}$, alt. $2000 \mathrm{~m}$, leg. PG, 11.08.2010 (POZNB 177, 21/2010); Važecká dolina valley, E part of lower part of the valley (Jamski Kocioł), on stone in peat-bog, $49.1538^{\circ} \mathrm{N}, 20.008^{\circ} \mathrm{E}$, alt. $1900 \mathrm{~m}$, leg. PG, 11.08.2010 (POZNB 200); 34UDV2746: Nefcerka valley, near W shore of Nižné Terianske pleso lake, alt. 1940 m, leg. PG, 2.08.2011 (POZNB 640); 34UDV2750: upper part of Zadna Tichá dolina valley, blocks of rock below Mt Tichý vrch, alt. $1770 \mathrm{~m}, 1785 \mathrm{~m}$, leg. PG, 29.07.2009 (POZNB 65, 25/2009); 34UDV2845: Furkotská dolina valley, near $\mathrm{N}$ shore of Nižné Wahlenbergovo pleso lake, blocks of rock, $49.16012^{\circ} \mathrm{N}, 20.02527^{\circ} \mathrm{E}$, alt. $2060 \mathrm{~m}$, leg. PG, 12.08.2010 (POZNB 205); 34UDV2946: Mlynická dolina valley, N slope of Bystrá lávka pass, alt. $2245 \mathrm{~m}$, leg. PG, 10.08.2008 (POZNB 114); 34UDV2947: upper part of Hlinská dolina valley, bottom plateau below Výsné Kôprovské sedlo pass, Salicetum herbaceae, alt. $1790 \mathrm{~m}$, leg. PG, 21.08.2006 (POZNB 146, 1566); 4UDV2949: Temnosmrečinská dolina valley, near route below Nižné Temnosmrečinské pleso lake, alt. $1670 \mathrm{~m}$, leg. PG, 22.07.2008 (POZNB 5/2008); Temnosmrečinská dolina valley, rocky outcrops between Nižné Temnosmrečinské pleso and Vyšné Temnosmrečinské pleso lakes, alt. 1700 m, 1705 m, leg. PG, 22.08.2006, 22.07.2008, 30.07.2009 (POZNB 293, 6/2008, 29/2009, 30/2009); 34UDV3046: upper, NE part of Mlynická dolina valley, blocks of rock, below rocky walls descending from Mt Velka Capia veža, alt. 2100 m, leg. PG, 11.08.2008 (POZNB 24/2008, 25/2008); 34UDV3048: Piargová dolinka valley, glacial cirque below Mt Čubrina, blocks of rock, $49.18583^{\circ} \mathrm{N}$, $20.04525^{\circ} \mathrm{E}, 49.18633^{\circ} \mathrm{N}, 20.04505^{\circ} \mathrm{E}, 49.18705^{\circ} \mathrm{N}$, $20.04422^{\circ} \mathrm{E}$, alt. $1865 \mathrm{~m}, 1870 \mathrm{~m}, 1875 \mathrm{~m}, 1880 \mathrm{~m}$, leg. PG, 22.08.2006, 22.07.2008, 28.07.2009 (POZNB 45/2006, 7/2008, 8/2008, 10/2008, 11/2008, 18/2009, 19/2009, 21/2009); 34UDV3148: Hincova dolina valley, rocky outcrops near NW shore of
Vel'ké Hincovo pleso lake, alt. 1940 m, leg. PG, 12.08.2008 (POZNB 28/2008); 34UDV3344: Mengusovská dolina valley, near route from Popradské pleso lake to Mt Ostrva, alt. $1675 \mathrm{~m}$, leg. PG, 10.08.2010 (POZNB 211); 34UDV3348: Spádová dolinka valley, SW part of the glacial cirque, blocks of rock below Mt Malé Rysy, alt. 2020 m, leg. PG, 25.08.2009 (POZNB 65/2009); 34UDV3349: Žabia Bielovodská dolina valley, blocks of rock above $S$ shore of Vyšné Žabie Bielovodské pleso lake, alt. 1700 m, 1705 m, 1710 m, 1730 m, leg. PG, 15.08.2009, 19.09.2009 (POZNB 89, 140, 704, 39/2009, 79/2009); 34UDV3445: Zlomisková dolina valley, Kotlina Ladového plesa basin, rocky outcrops above S shore of Ladové pleso lake, below Lúčne sedlo pass, $49.16193^{\circ} \mathrm{N}, 20.10615^{\circ} \mathrm{E}$, alt. $1975 \mathrm{~m}$, leg. $\mathrm{PG}$, 5.08.2010 (POZNB 228); Zlomisková dolina valley, on sub-glacially eroded step of upper glacial cirque (Kotlina Ladového plesa basin), near stream, $49.16232^{\circ} \mathrm{N}, 20.10407^{\circ} \mathrm{E}, 49.1624^{\circ} \mathrm{N}, 20.1029^{\circ} \mathrm{E}$, alt. 1880 m, 1925 m, leg. PG, 9.08.2011 (POZNB 647, 650); Zlomisková dolina valley, Zlomisková roveň, $49.16037^{\circ} \mathrm{N}, 20.09912^{\circ} \mathrm{E}$, alt. $1825 \mathrm{~m}$, leg. PG, 9.08.2011 (POZNB 672); 34UDV3446: Rumanova dolinka valley, lower part of the valley, snowbed with Gnaphalium supinum, $49.17002^{\circ} \mathrm{N}, 20.10235^{\circ} \mathrm{E}$, alt. 2165 m, leg. PG, 3.08.2010 (POZNB 218); Zlomisková dolina valley, Kotlina Ladového plesa basin, near $\mathrm{W}$ shore of Ladové pleso lake, $49.16352^{\circ} \mathrm{N}$, $20.1049^{\circ} \mathrm{E}$, alt. $1930 \mathrm{~m}$, leg. PG, 6.08.2010 (POZNB 225); 34UDV3447: Ťažká dolina valley, upper glacial cirque, blocks of rock below Mt Ganek and Mt Vysoká, alt. 1990 m, leg. PG, 20.08.2009 (POZNB 57/2009); Ťažká dolina valley, upper glacial cirque, blocks of rock below Mt Kačacia veža, alt. 1805 m, $1820 \mathrm{~m}$, leg. PG, 24.08.2009 (POZNB 61/2009, 62/2009); Tažká dolina valley, upper glacial cirque, near E shore of Zmarzlé pleso lake, below rocky walls descending from Mt Prédná Pustá veža, alt. $1765 \mathrm{~m}$, leg. PG, 20.08.2009 (POZNB 59/2009); 34UDV3448: Tažká dolina valley, near $\mathrm{N}$ shore of Zmarzlé pleso lake, below rocky walls descending from Mt Prédná Pustá veža, alt. 1760 m, leg. PG, 20.08.2009 (POZNB 55/2009); upper part of Žabia Bielovodská dolina valley, below rocky walls between Mlynárovo sedlo and Mt Velký Žabi štít, alt. 1890 m, leg. PG, 19.08.2009 (POZNB 51/2009); 34UDV3449: Žabia Bielovodská dolina valley, blocks of rock near S and SE shore of Nižné Žabie Bielovodské pleso lake, alt. $1681 \mathrm{~m}$, 1675 m, leg. PG, 14.08.2009 (POZNB 34/2009, 35/2009); Žabia Bielovodská dolina valley, E from S shore of Nižné Žabie Bielovodské pleso lake, below rocky walls descending from Mt Prostredný Mlynár, alt. 1735 m, leg. PG, 16.08.2009 (POZNB 40/2009); 34UDV3546: Kačacia dolina valley, upper glacial cirque (Żelazny Ogród), blocks of rock below Východná Železná brána pass, alt. 2000 m, 2015 m, leg. PG, 16.09.2009 (POZNB 143, 144); 34UDV3547: 
Kačacia dolina valley, lower glacial cirque, alt. 1645 m, leg. PG, 22.08.2007 (POZNB 7/2007); 34UDV3548: Ťažká dolina valley, upper part of the lower glacial cirque, blocks of rock below Mt Pustá stráž, alt. 1775 m, leg. PG, 26.08.2009 (POZNB 69); 34UDV3647: Litvorová dolina valley, below rocky walls descending from Mt Litvorový štít, alt. $1835 \mathrm{~m}$, $1860 \mathrm{~m}$, leg. PG, 15.09.2009, 17.08.2006 (POZNB 37/2006, 72/2009); Litvorová dolina valley, near S shore of Litvorové pleso lake, $49.17643^{\circ} \mathrm{N}$, 20.12872 ${ }^{\circ}$ E, alt. $1860 \mathrm{~m}$, leg. PG, 16.09.2009 (POZNB 142); Svištová (Bielovodská) dolina valley, Vyšna Svištová roveň, $49.18063^{\circ} \mathrm{N}, 20.13553^{\circ} \mathrm{E}$, alt. 1985 m, leg. PG, 26.09.2011 (POZNB 473); Svištová (Bielovodská) dolina valley, Svištové plieska lakes, near $\mathrm{N}$ shore of the biggest lake, alt. $1925 \mathrm{~m}$, leg. PG, 26.09.2011 (POZNB 488); 34UDV3747: upper part of Svištová (Bielovodská) dolina valley, blocks of rock below Zmarznutý kotol basin, $49.17915^{\circ} \mathrm{N}$, $20.13968^{\circ} \mathrm{E}$, alt. $2025 \mathrm{~m}$, leg. PG, 26.09.2011 (POZNB 485); 34UDV3848: Rovienky valley, Zadné Rovienky, $49.18747^{\circ} \mathrm{N}, 20.15157^{\circ} \mathrm{E}$, alt. $1925 \mathrm{~m}$, leg. PG, 25.09.2011 (POZNB 497); 34UDV3946: Veĺka Studená dolina valley, Dolinka nad Vareškovým Plesom valley, alt. $1845 \mathrm{~m}, 1850 \mathrm{~m}, 1870 \mathrm{~m}$, leg. PG, 23.08.2007 (POZNB 51, 9/2007, 10/2007); 34UDV3947: Veĺka Studená dolina valley, rocky walls near stream between Dlhé pleso and Vareškové pleso lake, alt. 1815 m, leg. PG, 23.08.2007 (POZNB 14/2007, 15/2007); Velka Studená dolina valley, Zbojnícky spád, alt. $1790 \mathrm{~m}$, leg. PG, 11.08.2011 (POZNB 580); 34UDV3948: Zadná Javorová dolina valley, glacial cirque below Mt Ostrý štít, alt. 2125 $\mathrm{m}$, leg. PG, 16.08.2006 (POZNB 28/2006); 34UDV3950: Suchá (Javorová) dolina valley, glacial cirque in upper part of the valley, alt. $1835 \mathrm{~m}$, leg. PG, 18.08.2006 (POZNB 39/2006); 34UDV4050: Čierna Javorová dolina valley, upper glacial cirque (Čierne mlaki), $49.2084^{\circ} \mathrm{N}, 20.18747^{\circ} \mathrm{E}$, alt. $1940 \mathrm{~m}$, leg. PG, 26.08.2010 (POZNB 163); 34UDV4051: Čierna Javorová dolina valley, Čierna záhrada, blocks of rock, near Czarna Siklawa stream, $49.21018^{\circ} \mathrm{N}$, $20.17982^{\circ} \mathrm{E}$, alt. $1635 \mathrm{~m}$, leg. PG, 25.08.2010 (POZNB 165); Čierna Javorová dolina valley, Čierne mlaki, on rocks dripping with water, $49.20945^{\circ} \mathrm{N}$, 20.1887E, alt. $1910 \mathrm{~m}$, leg. PG, 26.08.2010 (POZNB 171); 34UDV4149: Kotlina Piatich Spišských Plies valley, blocks of rock E from Prostredné Spišské pleso lake, alt. $2035 \mathrm{~m}$, leg. PG, 29.08.2007 (POZNB 25/2007); 34UDV4150: Čierna Javorová dolina valley, Czarny Bandzioch, $49.20782^{\circ} \mathrm{N}, 20.19433^{\circ} \mathrm{E}$, alt. 2050 m, leg. PG, 26.08.2010 (POZNB 28/2010); Čierna Javorová dolina valley, Czarny Bandzioch, near Čierny potok stream, $49.20842^{\circ} \mathrm{N}, 20.19167^{\circ} \mathrm{E}$, alt. 1995 m, leg. PG, 26.08.2010 (POZNB 29/2010); 34UDV4151: Kolová dolina valley, upper part of Kotlinka pod Kolovym sedlom basin, below Kolové sedlo pass, $49.21433^{\circ} \mathrm{N}, 20.20125^{\circ} \mathrm{E}$, alt. $2095 \mathrm{~m}$, leg. PG, 1.09.2011 (POZNB 464); 34UDV4152: Kolová dolina valley, above and SEE from Kolové pleso lake, below rocky walls descending from Hrebeň Kolových veži ridge, $49.21843^{\circ} \mathrm{N}, 20.19438^{\circ} \mathrm{E}$, alt. $1645 \mathrm{~m}$, leg. PG, 28.08.2010 (POZNB 257); Kolová dolina valley, near $\mathrm{N}$ shore of Kolové pleso lake, peat-bog, $49.22097^{\circ} \mathrm{N}, 20.19255^{\circ} \mathrm{E}$, alt. 1565 $\mathrm{m}$, leg. PG, 28.08.2010 (POZNB 229, 253); 34UDV4250: upper part of Vel'ká Zmrzlá dolina valley, Barania kotlina basin, $49.20268^{\circ} \mathrm{N}, 20.20448^{\circ} \mathrm{E}$, alt. 2085 m, leg. PG, 27.09.2011 (POZNB 542); 34UDV4251: Cervena dolina valley, near Červené pleso lake, alt. $1815 \mathrm{~m}$, leg. PG, 25.09.2007 (POZNB 32/2007, 33/2007); 34UDV4252: Dolina Bielych plies valley, alt. $1750 \mathrm{~m}, 1840 \mathrm{~m}$, leg. PG, 26.09.2007 (POZNB 34/2007, 36/2007); 34UDV4350: Dolina Kežmarskej Bielej Vody valley, near S shore of Zelené pleso lake, alt. $1541 \mathrm{~m}$, leg. PG, 21.08.2009 (POZNB 71); 34UDV4450: N slope of Mt Veĺká Svištovka, rocky crevices, alt. 1975 m, leg. PG, 30.09.2006 (POZNB 21).

Slovakia, Western Tatra Mountains: 34UDV0551: Jalovecká dolina valley, E part of Hlboká dolina valley (Vrece), below rocky walls descending from Mt Pachol'a, alt. 1810 m, 1815 m, leg. PG, 20.07.2008 (POZNB 2/2008, 3/2008); 34UDV0751: Roháčska dolina valley, blocks of rock near Horne Roháčske pleso lake, alt. $1720 \mathrm{~m}, 1755 \mathrm{~m}$, leg. PG, 24.07.2006 (POZNB 24, 22/2006); Spálená dolina valley, near route to Roháčske plesa lakes, alt. 1600 m, leg. PG, 24.07.2006 (POZNB 77); Spálená dolina valley, rocky outcrops near route, above Rázcestie k Roháčskym Plesám, alt. 1530 m, leg. PG, 24.07.2006 (POZNB 20/2006); 34UDV0849: Žiarska dolina valley, near Pliesko pod Ziarskom Sedlom lake, alt. $1835 \mathrm{~m}$, leg. PG, 20.07.2006 (POZNB 25); 34UDV0950: Zadná Jamnícka dolina valley (Kocioł Jamnickich Stawów), near Jamnícke Pleso Vyšné lake, alt. $1830 \mathrm{~m}$, leg. PG, 25.08.2008 (POZNB 132); 34UDV0953: Lataná dolina valley, below Zábrat pass, alt. $1420 \mathrm{~m}$, leg. PG, 18.07.2006 (POZNB 360); 34UDV1050: Jamnícka dolina valley, near $\mathrm{W}$ shore of Jamnícke Nižne pleso lake, alt. 1720 m, leg. PG, 25.08.2008 (POZNB 36/2008); 34UDV1250: Račkova dolina valley, Račkov Zadok, blocks of rock near Vyšné Račkove pleso lake, alt. 1715 m, leg. PG, 21.07.2009 (POZNB 3/2009); Račkova dolina valley, Račkov Zadok, glacial cirque below Mt Jakubiná, alt. $1845 \mathrm{~m}$, leg. PG, 21.07.2009 (POZNB 2/2009); 34UDV1649: Kamenistá dolina valley, glacial cirque below Mt Blyšt, alt. 1845 m, leg. PG, 29.08.2008 (POZNB 39/2008).

Poland, High Tatra Mountains: 34UDV2752: Dolina Gąsienicowa valley, glacial cirque below Mt Świnica, above Czerwone Stawki lakes, Grzędy, below rocky walls descending from Mt Skrajna Turnia, alt. 1870 m, leg. PG, 20.08.2005 (POZNB 19/2005); Dolina Gąsienicowa valley, glacial cirque below Mt Świ- 
nica, rocky outcrops SE from Zadni Staw Gasienicowy lake, alt. 1875 m, leg. PG, 20.08.2005 (POZNB 25/2005); 34UDV2753: Dolina Gąsienicowa valley, near S shore of Długi Staw lake, Salicetum herbaceae, $49.22595^{\circ} \mathrm{N}, 20.00882^{\circ} \mathrm{E}$, alt. $1780 \mathrm{~m}$, leg. PG, 20.09.2010 (POZNB 276); Dolina Gąsienicowa valley, near Czerwone Stawki lakes, $49.22743^{\circ} \mathrm{N}$, $20.00405^{\circ} \mathrm{E}$, alt. $1680 \mathrm{~m}$, leg. PG, 15.08.2013 (POZNB 1956); 34UDV2850: Dolina Pięciu Stawów Polskich valley, below Kotelnica ridge, above Czarny Staw Polski lake, alt. 1835 m, leg. PG, 27.07.2005 (POZNB 16/2005); 34UDV2851: Dolina Pięciu Stawów Polskich valley, at the mouth of Dolinka Pusta valley, $49.21063^{\circ} \mathrm{N}, 20.0247^{\circ} \mathrm{E}$, alt. $1805 \mathrm{~m}$, leg. PG, 24.08.2012 (POZNB 1766); 34UDV2852: Dolina Gąsienicowa valley, $S$ and above Zmarzły Staw Gąsienicowy lake, blocks of rock, alt. $1800 \mathrm{~m}$, 1845 m, leg. PG, 1.08.2003, 2.08.2003, 4.08.2004, 21.08.2005 (POZNB 302, 981, 99/2003, 29/2005); Dolina Gąsienicowa valley, at the mouth of Zawratowy Żleb gully, alt. 1955 m, leg. PG, 1.08.2003 (POZNB 106/2003); Dolina Gąsienicowa valley, on the sub-glacially eroded step of Dolinka Kozia valley, alt. $1810 \mathrm{~m}$, leg. PG, 21.08.2005 (POZNB 26/2005); 34UDV2853: Dolina Gąsienicowa valley, above NW shore of Czarny Staw Gąsienicowy lake, alt. 1680 m, leg. PG, 31.07.2003 (POZNB 95/2003); 34UDV2950: Dolina Pięciu Stawów Polskich valley, below Czarna Ławka pass, alt. $1870 \mathrm{~m}$, leg. PG, 27.07.2005 (POZNB 13/2005); 34UDV2953: Dolina Pańszczycy valley, below rocky walls descending from Pańszczycka Przełęcz pass, alt. 1985 m, leg. PG, 3.08.2003 (POZNB 115/2003); 34UDV3049: Dolina za Mnichem valley, below rocky walls descending from Mt Zadni Mnich, alt. 1980 m, leg. PG, 17.08.2004 (POZNB 23/2004); Dolina za Mnichem valley, below rocky walls descending from $\mathrm{Mt} \mathrm{Cu}-$ bryna, alt. $2000 \mathrm{~m}$, leg. PG, 16.08.2004 (POZNB 20/2004); Dolina za Mnichem valley, rocky rubble filling the valley bottom, alt. $1790 \mathrm{~m}, 1795 \mathrm{~m}$, leg. PG, 20.09.2005, 27.09.2007 (POZNB 117, 37/2007); 34UDV3050: Dolina Pięciu Stawów Polskich valley, Brzuchaty Piarg scree, below rocky walls descending from Mt Miedziane, alt. 1905 m, leg. PG, 25.07.2005 (POZNB 5/2005); 34UDV3151: Dolina Pięciu Stawów Polskich valley, Dolinka za Niedźwiedziem valley, alt. $1785 \mathrm{~m}$, leg. PG, 26.07.2005 (POZNB 7/2005); 34UDV3248: below Mt Kazalnica, rocks near route to Mięguszowiecka Przełęcz pod Chłopkiem pass, $49.18645^{\circ} \mathrm{N}, 20.06803^{\circ} \mathrm{E}$, alt. $1975 \mathrm{~m}$, leg. PG, 2.09.2014 (POZNB 1915); Dolina Rybiego Potoku valley, rocky outcrops above Czarny Staw pod Rysami lake, alt. 1715 m, leg. PG, 18.08.2004 (POZNB 24/2004).

Poland, Western Tatra Mountains: 34UdV0951: Mt Wołowiec, below the summit, alt. $2015 \mathrm{~m}$, leg. PG, 25.07.2009 (POZNB 128); 34UDV1150: Dolina Jarząbcza valley, below rocky walls descending from
Niska Przełęcz pass, alt. 1730 m, leg. PG, 9.08.2004 (POZNB 3/2004); 34UDV1251: Dolina Jarząbcza valley, NW slope of Mt Kończysty Wierch (Jarząbczy Kopieniec), leg. PG, 28.09.2003 (POZNB 148, 346); 34UDV1550: Ornak ridge, near Siwa Przełęcz pass, alt. 1770 m, leg. PG, 24.07.2009 (POZNB 13/2009).

\section{Diplophyllum taxifolium (Wahlenb.) Dumort.}

Slovakia, High Tatra Mountains: 34UDV2745: Važecká dolina valley, block of rock near SW shore of Krivánske Zelené pleso lake, $49.15792^{\circ} \mathrm{N}$, $20.00877^{\circ} \mathrm{E}$, alt. $2000 \mathrm{~m}$, leg. PG, 11.08.2010 (POZNB 204); 34UDV2949: Temnosmrečinská dolina valley, rocky outcrops near route, below Nižné Temnosmrečinské pleso lake, alt. $1670 \mathrm{~m}$, leg. PG, 30.07.2009 (POZNB 26/2009); 34UDV3046: Mlynická dolina valley, blocks of rock above $S$ shore of Vyšné Kozie pleso lake, alt. 2110 m, leg. PG, 11.08.2008 (POZNB 22/2008); 34UDV3048: Piargová dolinka valley, glacial cirque below Mt Čubrina, blocks of rock, $49.18583^{\circ} \mathrm{N}, 20.04525^{\circ} \mathrm{E}$, alt. $1880 \mathrm{~m}$, leg. PG, 22.07.2008 (POZNB 11/2008); 34UDV3346: Drača dolinka valley, blocks of rock above Drače pleso lake, $49.16503^{\circ} \mathrm{N}, 20.08898^{\circ} \mathrm{E}$, alt. $2050 \mathrm{~m}$, leg. PG, 5.08.2010 (POZNB 193); 34UDV3349: Žabia Bielovodská dolina valley, blocks of rock above $\mathrm{S}$ shore of Vyšné Žabie Bielovodské pleso lake, alt. $1705 \mathrm{~m}$, leg. PG, 19.09.2009 (POZNB 79/2009); 34UDV3445: Zlomisková dolina valley, Kotlina Ladového plesa basin, rocky outcrops above $\mathrm{S}$ shore of Ladové pleso lake, below Lúčne sedlo pass, $49.16208^{\circ} \mathrm{N}$, 20.10652 ${ }^{\circ}$ E, alt. $1980 \mathrm{~m}$, leg. PG, 5.08.2010 (POZNB 15/2010); 34UDV3547: Kačacia dolina valley, gully descending from Mt Zlobivá, alt. 1700 m, leg. PG, 22.08.2007 (POZNB 6/2007); Kačacia dolina valley, lower glacial cirque, alt. 1645 m, leg. PG, 22.08.2007 (POZNB 7/2007); Kačacia dolina valley, upper (SE) part of lower glacial cirque (Gierlachowskie Spady), alt. $1750 \mathrm{~m}$, leg. PG, 16.09.2009 (POZNB 76/2009); 34UDV3548: Tažká dolina valley, upper part of the lower glacial cirque, blocks of rock below Mt Pustá stráž, alt. $1810 \mathrm{~m}$, leg. PG, 24.08.2009 (POZNB 63/2009); 34UDV3747: Svištová (Bielovodská) dolina valley, Zmarznutý kotol basin, alt. 2060 m, leg. PG, 1.08.2006 (POZNB 27/2006); 34UDV3848: Rovienky valley, Zadné Rovienky, $49.18747^{\circ} \mathrm{N}$, $20.15157^{\circ} \mathrm{E}$, alt. $1925 \mathrm{~m}$, leg. PG, 25.09.2011 (POZNB 497); 34UDV3947: Veĺka Studená dolina valley, rocky walls near stream between Dlhé pleso and Vareškové pleso lakes, alt. $1815 \mathrm{~m}$, leg. PG, 23.08.2007 (POZNB 15/2007); 34UDV4050: Čierna Javorová dolina valley, Čierna záhrada, rocky walls at the mouth of Ladova dolinka valley, $49.20685^{\circ} \mathrm{N}$, $20.17762^{\circ} \mathrm{E}$, alt. $1660 \mathrm{~m}$, leg. PG, 25.08.2010 (POZNB 24/2010); 34UDV4250: Vel'ká Zmrzlá dolina valley, blocks of rock above sub-glacially eroded step, $49.20448^{\circ} \mathrm{N}, 20.20943^{\circ} \mathrm{E}$, alt. $1910 \mathrm{~m}$, leg. PG, 27.09.2011 (POZNB 551); 34UDV4251: Červena 
dolina valley, blocks of rock near Belasé pleso lake, alt. 1865 m, leg. PG, 25.09.2007 (POZNB 31/2007); 34UDV4252: Dolina Bielych plies valley, alt. $1750 \mathrm{~m}$, 1780 m, leg. PG, 26.09.2007 (POZNB 337, 35/2007). Slovakia, Western Tatra Mountains: 34UDV0453: Roháčska dolina valley, glacial cirque below $\mathrm{Mt}$ Brestová (from the north side), alt. $1720 \mathrm{~m}, 1725$ m, 1755 m, leg. PG, 23.07.2006 (POZNB 15/2006, 16/2006, 18/2006); 34UDV0551: Jalovecká dolina valley, E part of Hlboká dolina valley (Vrece), below rocky walls descending from Mt Pachol'a, $49.2054^{\circ} \mathrm{N}$, 19.69722 ${ }^{\circ}$ E, alt. $1770 \mathrm{~m}$, leg. PG, 17.07.2008 (POZNB 1/2008); 34UDV1050: Zadná Jamnícka dolina valley (Kocioł Jamnickich Stawów), above Jamnícke Nižne pleso lake, alt. 1760 m, leg. PG, 25.08.2008 (POZNB 30); 34UDV1648: Kobyla ridge (SE from Mt Bystrá), rocky outcrops near route to Bystrá dolina valley, $49.18053^{\circ} \mathrm{N}, 19.84937^{\circ} \mathrm{E}, 49.18118^{\circ} \mathrm{N}, 19.84977^{\circ} \mathrm{E}$, alt. 1955 m, 1990 m, leg. PG, 26.08.2011 (POZNB 452, 676); 34UDV1649: Kamenistá dolina valley, glacial cirque below Mt Blyšt́, alt. 1845 m, leg. PG, 29.08.2008 (POZNB 39/2008).

Poland, High Tatra Mountains: 34UDV2752: Dolina Gąsienicowa valley, glacial cirque below Mt Świnica, above Czerwone Stawki lakes, Grzędy, below rocky walls descending from Mt Skrajna Turnia, alt. 1870 m, leg. PG, 20.08.2005 (POZNB 19/2005); Dolina Gąsienicowa valley, glacial cirque below Mt Świnica, rocky outcrops SE from Zadni Staw Gąsienicowy lake, alt. 1875 m, leg. PG, 20.08.2005 (POZNB 25/2005); 34UDV2851: Dolina Pięciu Stawów Polskich valley, above S shore of Zadni Staw Polski lake, alt. 1885 m, leg. PG, 28.07.2005 (POZNB 944); 34UDV2852: Dolina Gąsienicowa valley, below rocky walls descending from Mt Zmarzłe Czuby, alt. 1930 m, leg. PG, 4.08.2003 (POZNB 118/2003); Dolina Gąsienicowa valley, blocks of rock above Zmarzły Staw lake, alt. $1795 \mathrm{~m}, 1800 \mathrm{~m}$, leg. PG, 2.08.2003, 4.08.2004 (POZNB 931, 981, 1455); 34UDV2853: Dolina Gąsienicowa valley, near E shore of Czarny Staw Gąsienicowy lake, near route to Granaty ridge, alt. 1640 m, leg. PG, 31.07.2003 (POZNB 91/2003); Dolina Gąsienicowa valley, blocks of rock slighty above W shore of Czarny Staw Gąsienicowy lake, alt. 1650 m, leg. PG, 31.07.2003 (POZNB 92/2003, 93/2003, 94/2003); Dolina Gąsienicowa valley, above S shore of Czarny Staw Gąsienicowy lake, below rocky walls descending from Mt Kościelec, alt. 1720 m, leg. PG, 31.07.2003 (POZNB 97/2003); 34UDV3049: Dolina za Mnichem valley, rock boulders near Staw Staszica lake, alt. $1790 \mathrm{~m}$, leg. PG, 12.09.2004 (POZNB 1457); 34UDV3149: Dolina za Mnichem valley, rocky outcrops above Wyżnie Stawki Staszica lakes, alt. 1835 m, leg. PG, 20.09.2005 (POZNB 32/2005); 34UDV3150: Dolina Pięciu Stawów Polskich valley, below rocky walls descending from Marchwiczna Przełęcz pass, alt. 1950 m, leg. PG, 26.07.2005 (POZNB 11/2005).
Poland, Western Tatra Mountains: 34UDV1251: Dolina Jarząbcza valley, NW slope of Mt Kończysty Wierch (Jarząbczy Kopieniec), alt. 1700 m, leg. PG, 28.09.2003, 10.08.2004 (POZNB 148, 8/2004); 34UDV1450: Dolina Starorobociańska valley, Zadnie Zagony, alt. 1700 m, leg. PG, 11.08.2004 (POZNB $12 / 2004,13 / 2004)$.

Fuscocephaloziopsis albescens (Hook.) Váňa et L. Söderstr. [= Pleurocladula albescens (Hook.) Grolle] Slovakia, High Tatra Mountains: 34UDV2745: Važecká dolina valley, blocks of rock near W shore of Krivánske Zelené pleso lake, $49.15888^{\circ} \mathrm{N}, 20.00895^{\circ} \mathrm{E}$, $49.15952^{\circ} \mathrm{N}, 20.00887^{\circ} \mathrm{E}$, alt. $2000 \mathrm{~m}, 2005 \mathrm{~m}$, leg. PG, 11.08.2010 (POZNB 217, 227); 34UDV2746: Nefcerka valley, near W shore of Nižné Terianske pleso lake, alt. $1940 \mathrm{~m}$, leg. PG, 2.08.2011 (POZNB 640); 34UDV2750: Zadná Tichá dolina valley, blocks of rock below Mt Tichý vrch, alt. 1785 m, leg. PG, 29.07.2009 (POZNB 65); 34UDV2846: Nefcerka valley, near SW shore of Vyšné Terianské pleso lake, $49.16768^{\circ} \mathrm{N}, 20.02032^{\circ} \mathrm{E}$, alt. $2085 \mathrm{~m}$, leg. PG, 3.08.2011 (POZNB 607); Nefcerka valley, blocks of rock $\mathrm{E}$ from $\mathrm{E}$ shore of Nižné Terianske pleso lake, $49.16727^{\circ} \mathrm{N}, 20.01613^{\circ} \mathrm{E}$, alt. $2035 \mathrm{~m}$, leg. PG, 2.08.2012 (POZNB 1326); Nefcerka valley, the highest glacial cirque, below Furkotské sedlo pass, $49.16905^{\circ} \mathrm{N}, 20.02283^{\circ} \mathrm{E}$, alt. $2150 \mathrm{~m}$, leg. $\mathrm{PG}$, 3.08.2011 (POZNB 1982); 34UDV2945: Mlynická dolina valley, blocks of rock below Mt Malé Solisko, alt. 2015 m, 2030 m, leg. PG, 15.08.2008 (POZNB 105, 34/2008); 34UDV2946: Furkotská dolina valley, blocks of rock near SE shore of Vyšné Wahlenbergovo pleso lake, alt. $2100 \mathrm{~m}, 2125 \mathrm{~m}, 2130 \mathrm{~m}$, leg. PG, 8.08.2008 (POZNB 86, 13/2008, 14/2008, 15/2008); Mlynická dolina valley, nothern slope of Bystrá lávka pass, alt. 2285 m, leg. PG, 10.08.2008 (POZNB 17/2008); 34UDV3045: Mlynická dolina valley, above $S$ shore of Nižne Kozie pleso lake, alt. 1945 m, leg. PG, 11.08.2008 (POZNB 26/2008); Mlynická dolina valley, near Volie pliesko lake, alt. 1915 m, leg. PG, 11.08.2008 (POZNB 27/2008); Mlynická dolina valley, below rocky walls descending from Mt Malé Solisko, alt. 1945 m, leg. PG, 15.08.2008 (POZNB 33/2008); 34UDV3046: Mlynická dolina valley, blocks of rock above $S$ shore of Vyšné Kozie pleso lake, alt. $2110 \mathrm{~m}$, leg. PG, 11.08.2008 (POZNB 22/2008); upper, NE part of Mlynická dolina valley, blocks of rock, below rocky walls descending from Mt Vel'ka Capia veža, alt. 2100 m, leg. PG, 11.08.2008 (POZNB 24/2008, 25/2008); 34UDV3047: Hlinská dolina valley, bottom plateau below Výsné Kôprovské sedlo pass, alt. $1775 \mathrm{~m}, 1790 \mathrm{~m}$, leg. PG, 21.08.2006 (POZNB 146, 147); Hlinská dolina valley, below Výsné Kôprovské sedlo pass, alt. 1950 m, leg. PG, 21.08.2006 (POZNB 41/2006, 42/2006, 43/2006); 34UDV3048: Piargová dolinka valley, glacial cirque below Mt Čubrina, blocks of rock, $49.18657^{\circ} \mathrm{N}$, 
$20.04512^{\circ} \mathrm{E}, 49.18633^{\circ} \mathrm{N}, 20.04505^{\circ} \mathrm{E}, 49.18583^{\circ} \mathrm{N}$, $20.04525^{\circ} \mathrm{E}, 49.18757^{\circ} \mathrm{N}, 20.0453^{\circ} \mathrm{E}$, alt. $1875 \mathrm{~m}$, $1880 \mathrm{~m}, 1885 \mathrm{~m}$, leg. PG, 22.07.2008 (POZNB 9/2008, 10/2008, 11/2008, 12/2008); Piargová dolinka valley, glacial cirque below Mt Čubrina, blocks of rock, alt. $1860 \mathrm{~m}, 1865 \mathrm{~m}, 1875 \mathrm{~m}, 1880 \mathrm{~m}$, leg. PG, 22.08.2006, 22.07.2008, 28.07.2009 (POZNB 20, 18/2009, 20/2009, 21/2009, 22/2009, 23/2009, 24/2009, 44/2006, 45/2006); 34UDV3148: Hincova dolina valley, rocky outcrops near $\mathrm{N}$ shore of Velké Hincovo pleso lake, alt. 1940 m, leg. PG, 12.08.2008 (POZNB 29/2008); 34UDV3247: Žabia Mengusovská dolina valley, Kotlina Žabich plies basin, blocks of rock N and above Velké Žabie Pleso Mengusovské lake, alt. $1935 \mathrm{~m}$, leg. PG, 13.08.2008 (POZNB 31/2008); 34UDV3346: Drača dolinka valley, blocks of rock above Drače pleso lake, $49.16503^{\circ} \mathrm{N}$, $20.08898^{\circ} \mathrm{E}, 49.16772^{\circ} \mathrm{N}, 20.0865^{\circ} \mathrm{E}$, alt. $2050 \mathrm{~m}$, 2075 m, leg. PG, 5.08.2010 (POZNB 193, 14/2010); 34UDV3349: Žabia Bielovodská dolina valley, snowbed near SE shore of Vyšné Žabie Bielovodské pleso lake, alt. $1700 \mathrm{~m}, 1710 \mathrm{~m}$, leg. PG, 15.08.2009, 19.09.2009 (POZNB 56, 140); Žabia Bielovodská dolina valley, blocks of rock above $S$ shore of Vyšné Žabie Bielovodské pleso lake, alt. 1705 m, 1730 m, leg. PG, 15.08.2009, 19.09.2009 (POZNB 37/2009, 38/2009, 39/2009, 79/2009); 34UDV3445: Zlomisková dolina valley, Kotlina Ladového plesa basin, rocky outcrops above S shore of Ladové pleso lake, below Lúčne sedlo pass, $49.16193^{\circ} \mathrm{N}, 20.10615^{\circ} \mathrm{E}$, $49.16208^{\circ} \mathrm{N}, 20.10652^{\circ} \mathrm{E}, 49.16158^{\circ} \mathrm{N}, 20.10633^{\circ} \mathrm{E}$, alt. 1975 m, 1980 m, 2005 m, leg. PG, 5.08.2010 (POZNB 228, 15/2010, 16/2010); Zlomisková dolina valley, Kotlina Ladového plesa basin, near SE shore of Ladové pleso lake, $49.16285^{\circ} \mathrm{N}, 20.10755^{\circ} \mathrm{E}$, alt. $1930 \mathrm{~m}$, leg. PG, 6.08.2010 (POZNB 19/2010); 34UDV3446: Rumanova dolinka valley, lower part of the valley, snowbed with Gnaphalium supinum, $49.17002^{\circ} \mathrm{N}, 20.10235^{\circ} \mathrm{E}$, alt. $2165 \mathrm{~m}$, leg. PG, 3.08.2010 (POZNB 218); Zlomisková dolina valley, Kotlina Ladového plesa basin, near $\mathrm{W}$ shore of Ladové pleso lake, $49.16352^{\circ} \mathrm{N}, 20.1049^{\circ} \mathrm{E}$, alt. 1930 $\mathrm{m}$, leg. PG, 6.08.2010 (POZNB 225); Rumanova dolinka valley, near NE shore of Rumanovo pleso lake, $49.16842^{\circ} \mathrm{N}, 20.0997^{\circ} \mathrm{E}$, alt. $2150 \mathrm{~m}$, leg. PG, 3.08.2010 (POZNB 226); 34UDV3447: Ťažká dolina valley, upper glacial cirque, blocks of rock above $S$ shore of Zmarzlé pleso lake, alt. $1805 \mathrm{~m}$, leg. PG, 20.08.2009 (POZNB 56/2009); Ťažká dolina valley, upper glacial cirque, blocks of rock below Mt Kačacia veža, alt. $1805 \mathrm{~m}$, leg. PG, 24.08.2009 (POZNB 61/2009); 34UDV3448: Ťažká dolina valley, upper glacial cirque, near $\mathrm{N}$ shore of Zmarzlé pleso lake, below rocky walls descending from Mt Prédná Pustá veža, alt. 1760 m, leg. PG, 20.08.2009 (POZNB 87, 55/2009); Ťažká dolina valley, lower glacial cirque, above SE shore of Ťažké pleso lake, alt. $1670 \mathrm{~m}$, leg. PG, 24.08.2009 (POZNB 60/2009); Spádová dolinka valley, SW part of the glacial cirque, blocks of rock below rocky walls descending from Mt Malé Rysy, alt. 1995 m, leg. PG, 25.08.2009 (POZNB 66/2009); upper part of Žabia Bielovodská dolina valley, below rocky walls between Mlynárovo sedlo pass and $\mathrm{Mt}$ Velký Žabi štít, alt. $1885 \mathrm{~m}, 1920 \mathrm{~m}$, leg. PG, 17.08.2009, 19.08.2009 (POZNB 46/2009, 47/2009, 49/2009, 50/2009, 52/2009, 53/2009);34UDV3449: Žabia Bielovodská dolina valley, blocks of rock near S and SE shore of Nižné Žabie Bielovodské pleso lake, alt. $1675 \mathrm{~m}, 1681 \mathrm{~m}$, leg. PG, 14.08.2009 (POZNB 34/2009, 35/2009); Žabia Bielovodská dolina valley, E from S shore of Nižné Žabie Bielovodské pleso lake, below rocky walls descending from Mt Prostredný Mlynár, alt. 1735 m, leg. PG, 16.08.2009 (POZNB 40/2009, 41/2009); Žabia Bielovodská dolina valley, rocky walls NW from Mlynárovo sedlo pass, alt. $1920 \mathrm{~m}$, leg. PG, 16.08.2009 (POZNB 43/2009); 34UDV3544: Štôlska dolina valley, lower part of the valley, above Tatranská Magistrála route, blocks of rock, $49.14893^{\circ} \mathrm{N}, 20.11088^{\circ} \mathrm{E}$, alt. $1935 \mathrm{~m}$, leg. PG, 10.08.2010 (POZNB 174); 34UDV3545: Batizovská dolina valley, blocks of rock near S and SE shore of Pliesko pod Kostolikom lake, $49.1603^{\circ} \mathrm{N}$, $20.12135^{\circ} \mathrm{E}, 49.16117^{\circ} \mathrm{N}, 20.12187^{\circ} \mathrm{E}$, alt. $2045 \mathrm{~m}$, 2055 m, leg. PG, 1.08.2010 (POZNB 1/2010, 2/2010); 34UDV3546: Kačacia dolina valley, upper glacial cirque (Żelazny Ogród), blocks of rock below Východná Železná brána pass, alt. 1985 m, leg. PG, 16.09.2009 (POZNB 74/2009); 34UDV3547: Kačacia dolina valley, lower glacial cirque, alt. $1645 \mathrm{~m}$, leg. PG, 22.08.2007 (POZNB 7/2007); 34UDV3548: Ťažká dolina valley, lower glacial cirque, above SE shore of Ťažké pleso lake, alt. $1680 \mathrm{~m}$, leg. PG, 26.08.2009 (POZNB 110); Ťažká dolina valley, upper part of the lower glacial cirque, blocks of rock below Mt Pustá stráž, alt. $1810 \mathrm{~m}, 1815 \mathrm{~m}$, leg. PG, 24.08.2009, 26.08.2009 (POZNB 64/2009, 67/2009, 68/2009); 34UDV3647: Kačacia dolina valley, on the sub-glacially eroded step of Litvorová dolina valley, alt. 1685 m, leg. PG, 15.09.2009 (POZNB 141); Litvorová dolina valley, near S shore of Litvorové pleso lake, $49.17643^{\circ} \mathrm{N}, 20.12872^{\circ} \mathrm{E}$, alt. $1860 \mathrm{~m}$, leg. PG, 16.09.2009 (POZNB 142); Litvorová dolina valley, below rocky walls descending from Mt Litvorový štít, alt. $1835 \mathrm{~m}$, leg. PG, 15.09.2009 (POZNB 72/2009); Litvorová dolina valley, rocky outcrops E from Litvorové pleso lake, alt. $1880 \mathrm{~m}, 1940 \mathrm{~m}$, leg. PG, 17.08.2006 (POZNB 34/2006, 36/2006); Svištová (Bielovodská) dolina valley, Vyšna Svištová roveň, $49.18063^{\circ} \mathrm{N}, 20.13553^{\circ} \mathrm{E}$, alt. $1985 \mathrm{~m}$, leg. PG, 26.09.2011 (POZNB 474); Svištová (Bielovodská) dolina valley, Svištové plieska lakes, near the biggest lake, alt. 1925 m, leg. PG, 26.09.2011 (POZNB 488); 34UDV3746: Velická dolina valley, Velická kotlina basin, blocks of rock near Vyšné Velické plieska lakes, $49.1723^{\circ} \mathrm{N}, 20.13588^{\circ} \mathrm{E}$, alt. $2125 \mathrm{~m}$, leg. PG, 8.08.2012 (POZNB 1145); 34UDV3747: Svištová 
(Bielovodská) dolina valley, Zmarznutý kotol basin, alt. 2035 m, 2065 m, leg. PG, 1.08.2006 (POZNB 24/2006, 25/2006); Svištová (Bielovodská) dolina valley, Zmarznutý kotol basin, $49.17898^{\circ} \mathrm{N}$, 20.13897E, alt. $2015 \mathrm{~m}$, leg. PG, 26.09.2011 (POZNB 479); Svištová (Bielovodská) dolina valley, Zmarznutý kotol basin, near Zmarznuté pleso lake, $49.17645^{\circ} \mathrm{N}$, 20.13918 E, alt. $2040 \mathrm{~m}$, leg. PG, 26.09.2011 (POZNB 482); 34UDV3848: Rovienky valley, Zadné Rovienky, $\quad 49.18747^{\circ} \mathrm{N}, \quad 20.15157^{\circ} \mathrm{E}, \quad 49.18767^{\circ} \mathrm{N}$ $20.15355^{\circ} \mathrm{E}, 49.18745^{\circ} \mathrm{N}, 20.15342^{\circ} \mathrm{E}$, alt. $1925 \mathrm{~m}$, 1930 m, 1950 m, leg. PG, 25.09.2011 (POZNB 497, 501, 604); 34UDV3946: Veĺka Studená dolina valley, Dolinka nad Vareškovým Plesom valley, alt. 1845 m, $1850 \mathrm{~m}, 1860 \mathrm{~m}, 1870 \mathrm{~m}$, leg. PG, 23.08.2007 (POZNB 51, 9/2007, 10/2007, 12/2007, 13/2007); 34UDV3947: Veĺka Studená dolina valley, blocks of rock W from Zbojnícka chata hut, alt. $1935 \mathrm{~m}$, leg. PG, 24.08.2007 (POZNB 8); Veĺka Studená dolina valley, rocky walls near stream between Dlhé pleso and Vareškové pleso lakes, alt. $1815 \mathrm{~m}$, leg. PG, 23.08.2007 (POZNB 15/2007); 34UDV3948: Zadná Javorová dolina valley, glacial cirque below Mt Ostrý štít, alt. $2125 \mathrm{~m}$, leg. PG, 16.08.2006 (POZNB 28/2006, 29/2006); Zadná Javorová dolina valley, glacial cirque below rocky walls between Mt Ostrý štít and Mt Javorový štít, alt. 2085 m, leg. PG, 16.08.2006 (POZNB 32/2006); 34UDV3949: Zadná Javorová dolina valley, Sobkova úboč, blocks of rock, alt. $1985 \mathrm{~m}$, leg. PG, 21.08.2007 (POZNB 94); 34UDV3950: Suchá (Javorová) dolina valley, bank near Suchá voda stream, alt. $1730 \mathrm{~m}$, leg. PG, 18.08.2006 (POZNB 678); Suchá (Javorová) dolina valley, glacial cirque in upper part of the valley, alt. $1930 \mathrm{~m}$, leg. PG, 18.08.2006 (POZNB 18); 34UDV4048: Veĺka Studená dolina valley, Strelecké polia, $49.18543^{\circ} \mathrm{N}, 20.17883^{\circ} \mathrm{E}$, alt. $2105 \mathrm{~m}$, leg. PG, 11.08.2011 (POZNB 661); 34UDV4049: Zadná Javorová dolina valley, below rocky walls descending from Mt Ostrí štít, alt. 2130 m, leg. PG, 16.08.2006 (POZNB 75); Zadná Javorová dolina valley, below Sedielko pass, alt. $2110 \mathrm{~m}$, leg. PG, 16.08.2006 (POZNB 78); 34UDV4050: Čierna Javorová dolina valley, upper glacial cirque (Čierne mlaki), $49.2084^{\circ} \mathrm{N}$, 20.18747 ${ }^{\circ}$ E, alt. $1940 \mathrm{~m}$, leg. PG, 26.08.2010 (POZNB 163); Čierna Javorová dolina valley, Čierne mlaki, blocks of rock below rocky walls descending from Hrebeň Snehových veži ridge, $49.2075^{\circ} \mathrm{N}$, 20.18718 ${ }^{\circ}$ E, alt. 1975 m, leg. PG, 26.08.2010 (POZNB 25/2010); 34UDV4051: Cierna Javorová dolina valley, Čierne mlaki, on rocks dripping with water, $49.20945^{\circ} \mathrm{N}, 20.1887^{\circ} \mathrm{E}$, alt. $1910 \mathrm{~m}$, leg. PG, 26.08.2010 (POZNB 171); 34UDV4148: upper part of Malá Studená dolina valley, alt. $1935 \mathrm{~m}$, leg. PG, 29.08.2007 (POZNB 30/2007); 34UDV4149: Kotlina Piatich Spišských Plies valley, above $\mathrm{W}$ shore of Prostredné Spišské pleso lake, alt. 2000 m, 2005 m, leg. PG, 27.08.2007 (POZNB 18/2007, 19/2007,
20/2007, 22/2007); Kotlina Piatich Spišských Plies valley, blocks of rock NW from Velké Spišské pleso lake, alt. $2070 \mathrm{~m}$, leg. PG, 27.08.2007 (POZNB 24/2007); 34UDV4150: Čierna Javorová dolina valley, Czarny Bandzioch, $49.20667^{\circ} \mathrm{N}, 20.19423^{\circ} \mathrm{E}$, alt. 2075 m, leg. PG, 26.08.2010 (POZNB 27/2010); 34UDV4151: Kolová dolina valley, upper part of Kotlinka pod Kolovym sedlom basin, below Kolové sedlo pass, $49.21433^{\circ} \mathrm{N}, 20.20125^{\circ} \mathrm{E}$, alt. $2095 \mathrm{~m}$, leg. PG, 1.09.2011 (POZNB 466); 34UDV4152: Kolová dolina valley, blocks of rock in upper part of the valley, $49.21825^{\circ} \mathrm{N}, 20.20028^{\circ} \mathrm{E}$, alt. $1820 \mathrm{~m}$, leg. PG, 1.09.2011 (POZNB 448); 34UDV4250: Vel'ká Zmrzlá dolina valley, blocks of rock above sub-glacially eroded step, $49.20448^{\circ} \mathrm{N}, 20.20943^{\circ} \mathrm{E}$, alt. $1910 \mathrm{~m}$, leg. PG, 27.09.2011 (POZNB 554); 34UDV4251: Červena dolina valley, near Červené pleso lake, alt. 1815 m, leg. PG, 25.09.2007 (POZNB 32/2007, 33/2007); 34UDV4252: Dolina Bielych plies valley, alt. $1750 \mathrm{~m}, 1840 \mathrm{~m}$, leg. PG, 26.09.2007 (POZNB 34/2007, 36/2007); 34UDV4450: N slope of Mt Veĺká Svištovka, Polytrichum-Sphagnum hummock, alt. 1900 m, leg. PG, 30.09.2006 (POZNB 19); N slope of Mt Veíká Svištovka, rocky crevices, alt. 1975 m, leg. PG, 30.09.2006 (POZNB 21).

Slovakia, Western Tatra Mountains: 34UDV0453: Roháčska dolina valley, glacial cirque below Mt Brestová (from the north side), alt. $1780 \mathrm{~m}$, leg. PG, 23.07.2006 (POZNB 17/2006); 34UDV0550: Spálená dolina valley, below Baníkovské sedlo pass, alt. 1855 m, leg. PG, 19.07.2006 (POZNB 23); 34UDV0751: Roháčska dolina valley, blocks of rock near Horne Roháčske pleso lake, alt. 1720 m, 1755 m, leg. PG, 24.07.2006 (POZNB 24, 22/2006); 34UDV0849: Žiarska dolina valley, near Pliesko pod Ziarskom Sedlom lake, alt. 1835 m, leg. PG, 20.07.2006 (POZNB 25); Žiarska dolina valley, Malé Závraty, glacial cirque below Žiarskie sedlo pass and Mt Smrek, alt. 1785 m, 1790 m, leg. PG, 20.07.2006 (POZNB 9/2006, 10/2006); 34UDV0949: snowbed on a ridge between Žiarskie sedlo pass and Mt Smrek, alt. 2025 m, leg. PG, 21.07.2006 (POZNB 13/2006, 14/2006); 34UDV1250: Račkova dolina valley, Račkov Zadok, glacial cirque below Mt Jakubiná, alt. 1810 m, 1830 $\mathrm{m}, 1845 \mathrm{~m}$, leg. PG, 21.07.2009 (POZNB 1/2009, 2/2009, 6/2004); Račkova dolina valley, Račkov Zadok, near W shore of Vyšné Račkove pleso lake, alt. 1730 m, leg. PG, 27.08.2011 (POZNB 455).

Poland, High Tatra Mountains: 34UDV2752: Dolina Gąsienicowa valley, glacial cirque below Mt Świnica, above Czerwone Stawki lakes, Grzędy, below rocky walls descending from Mt Skrajna Turnia, alt. 1875 m, leg. PG, 20.08.2005 (POZNB 20/2005); Dolina Gąsienicowa valley, glacial cirque below Mt Świnica, rocky outcrops SE from Zadni Staw Gąsienicowy lake, alt. 1875 m, leg. PG, 20.08.2005 (POZNB 25/2005); 34UDV2753: Dolina Gąsienicowa valley, near $S$ shore of Długi Staw Gąsienicowy 
lake, Salicetum herbaceae, $49.22595^{\circ} \mathrm{N}, 20.00882^{\circ} \mathrm{E}$, alt. $1780 \mathrm{~m}$, leg. PG, 20.09.2010 (POZNB 276); 34UDV2850: Dolina Pięciu Stawów Polskich valley, below Kotelnica ridge, above Czarny Staw Polski lake, alt. 1835 m, leg. PG, 27.07.2005 (POZNB 16/2005); 34UDV2851: Dolina Pięciu Stawów Polskich valley, sub-glacially eroded step of Dolinka Pusta valley, blocks of rock, $49.21427^{\circ} \mathrm{N}, 20.0227^{\circ} \mathrm{E}$, alt. 1960 m, leg. PG, 24.08.2012 (POZNB 1964); 34UDV2852: Dolina Gąsienicowa valley, near SW shore of Zmarzły Staw Gąsienicowy lake, Salicetum herbaceae, $49.22377^{\circ} \mathrm{N}, 20.02213^{\circ} \mathrm{E}$, alt. $1778 \mathrm{~m}$, leg. PG, 21.09.2010 (POZNB 232); Dolina Gąsienicowa valley, above Zmarzły Staw Gąsienicowy lake, blocks of rock, alt. 1800-1845 m, leg. PG, 2.08.2003, 21.08.2005 (POZNB 286, 288, 931, 29/2005, 30/2005); Dolina Gąsienicowa valley, SW and above from Zmarzły Staw Gąsienicowy lake, alt. $1800 \mathrm{~m}$, leg. PG, 1.08.2003 (POZNB 100/2003, 101/2003); Dolina Gąsienicowa valley, on the sub-glacially eroded step of Dolinka Kozia valley, alt. 1810 m, leg. PG (POZNB 26/2005); Dolina Gąsienicowa valley, below Zawratowy Żleb gully, alt. 1825-1840 m, leg. PG, 1.08.2003 (POZNB 103/2003, 104/2003, 107/2003); Dolina Gąsienicowa valley, at the mouth of Zawratowy Żleb gully, alt. $1955 \mathrm{~m}$, leg. PG, 1.08.2003 (POZNB 106/2003); Dolina Gąsienicowa valley, below rocky walls descending from $\mathrm{Mt}$ Zmarzłe Czuby, alt. 1930 m, leg. PG, 4.08.2003, (POZNB 117/2003, 118/2003); Dolina Gąsienicowa valley, below rocky walls descending from $\mathrm{Mt}$ Zmarzła Przełęcz pass, alt. 2015 m, leg. PG, 4.08.2003 (POZNB 120/2003, 121/2003); 34UDV2853: Dolina Gąsienicowa valley, glacial cirque below Mt Świnica, blocks of rock near Długi Staw Gąsienicowy lake, alt. $1845 \mathrm{~m}$, leg. PG, 20.08.2005 (POZNB 21/2005); 34UDV2950: Dolina Pięciu Stawów Polskich valley, below rocky walls descending from Mt Wyżni Liptowski Kostur, alt. 1940 m, 2040 m, leg. PG, 25.07.2005 (POZNB 1/2005, 2/2005); Dolina Pięciu Stawów Polskich valley, below Czarna Ławka pass, alt. $1870 \mathrm{~m}$, leg. PG, 27.07.2005 (POZNB 12/2005, 13/2005, 14/2005); 34UDV2952: Dolinka Kozia valley, bottom of plateau, alt. 1920 m, leg. PG, 21.08.2005 (POZNB 28/2005); upper part of Dolinka Kozia valley, below rocky walls descending from Orla Perć ridge, alt. 2050 m, 2055 m, leg. PG, 2.08.2003 (POZNB 108/2003, 109/2003); on the sub-glacially eroded step of Dolinka Kozia valley, alt. 1960 m, leg. PG, 31.08.2011 (POZNB 461); 34UDV2953: Dolina Pańszczycy valley, below rocky walls descending from Pańszczycka Przełęcz pass, alt. 1995 m, leg. PG, 3.08.2003 (POZNB 113/2003); 34UDV2959: Dolina Pięciu Stawów Polskich valley, blocks of rock below Mt Wyżni Liptowski Kostur, $49.20075^{\circ} \mathrm{N}, 20.02813^{\circ} \mathrm{E}$, alt. $1880 \mathrm{~m}$, leg. PG, 22.08.2012 (POZNB 1966); 34UDV3049: Dolina za Mnichem valley, near Wyżni Staw Staszica lake, alt. $1780 \mathrm{~m}$, leg. PG, 16.08.2004, 20.09.2004 (POZNB 26, 15/2004); Dolina za Mnichem valley, bottom of plateau, blocks of rock and near stream, alt. 1790-1820 m, leg. PG, 20.09.2005, 27.09.2007 (POZNB 116, 117); Dolina za Mnichem valley, below Mt Cubryna, snowbed among blocks of rock, alt. 1900-2000 m, leg. PG, 16.08.2004 (POZNB 150, 19/2004); Dolina za Mnichem valley, below rocky walls descending from Wrota Chałubińskiego pass, alt. $1850 \mathrm{~m}, 2000 \mathrm{~m}$, leg. PG, 16.08.2004 (POZNB $18 / 2004,21 / 2004)$; Dolina za Mnichem valley, below rocky walls descending from Mt Zadni Mnich, alt. 1980 m, leg. PG, 17.08.2004 (POZNB 23/2004); 34UDV3050: Dolina Pięciu Stawów Polskich valley, Czerwony Piarg scree, near route to Szpiglasowa Przełęcz pass, alt. 1955 m, leg. PG, 25.07.2005 (POZNB 4/2005); Dolina Pięciu Stawów Polskich valley, Brzuchaty Piarg scree, below rocky walls descending from Mt Miedziane, alt. 1905 m, leg. PG, 25.07.2005 (POZNB 5/2005); 34UDV3051: Dolina Pięciu Stawów Polskich valley, S from Przedni Staw Polski lake, alt. $1670 \mathrm{~m}$, leg. PG, 25.07.2005 (POZNB 6/2005); 34UDV3053: Dolina Waksmundzka valley, below Mt Wołoszyn, alt. $1995 \mathrm{~m}$, leg. PG, 22.08.2005 (POZNB 31/2005); 34UDV3149: Dolina za Mnichem valley, rocky outcrops above Wyżnie Stawki Staszica lakes, alt. 1825 m, 1835 m, leg. PG, 20.09.2005 (POZNB 32/2005, 33/2005); Dolina za Mnichem valley, peat-bog near Wyżnie Stawki Staszica lakes, alt. 1855 m, leg. PG, 28.08.2012 (POZNB 1497); 34UDV3151: Dolina Pięciu Stawów Polskich valley, Dolinka za Niedźwiedziem valley, alt. 1785 m, leg. PG, 26.07.2005 (POZNB 7/2005); Dolina Pięciu Stawów Polskich valley, below rocky walls descending from Marchwiczna Przełęcz pass, alt. 1810 m, 1815 m, 1900 m, leg. PG, 26.07.2005 (POZNB 8/2005, 9/2005, 10/2005); 34UDV3248: below Mięguszowiecka Przełęcz pod Chłopkiem pass, rocky crevices, alt. 2255 m, leg. PG, 2.09.2014 (POZNB 1904).

\section{Gymnocolea inflata (Huds.) Dumort.}

Slovakia, High Tatra Mountains: 34UDV2845: Furkotská dolina valley, near N shore of Nižne Wahlenbergovo pleso lake, $49.16012^{\circ} \mathrm{N}, 20.02527^{\circ} \mathrm{E}$, alt. 2055 m, leg. PG, 12.08.2010 (POZNB 269); 34UDV2846: Nefcerka valley, blocks of rock S from Vyšné Terianske pleso lake, $49.1658^{\circ} \mathrm{N}, 20.0198^{\circ} \mathrm{E}$, alt. 2125 m, leg. PG (POZNB 1971); 34UDV2850: Kobylia dolina valley, near E shore of Kobylie pleso lake, alt. $1740 \mathrm{~m}$, leg. PG, 29.07.2009 (POZNB 120); 34UDV2949: Temnosmrečinská dolina valley, between Nižné and Vyšné Temnosmrečinské pleso lakes, on stone near unmarked path, alt. $1710 \mathrm{~m}$, leg. PG, 22.07.2008 (POZNB 357); 34UDV3044: Mlynická dolina valley, rocky walls below Vodopád Skok waterfall, alt. 1705 m, leg. PG, 18.07.2011 (POZNB 576); 34UDV3446: Rumanova dolinka 
valley, blocks of rock near Vyšné Rumanovo pliesko lake, $49.17212^{\circ} \mathrm{N}, 20.10102^{\circ} \mathrm{E}$, alt. $2135 \mathrm{~m}$, leg. $\mathrm{PG}$, 9.08.2011 (POZNB 571); S part of Rumanova dolinka valley, $49.1657^{\circ} \mathrm{N}, 20.10428^{\circ} \mathrm{E}$, alt. $2045 \mathrm{~m}$, leg. PG, 9.08.2011 (POZNB 613); 34UDV3545: S slope of Mt Končistá, $49.15527^{\circ} \mathrm{N}, 20.11467^{\circ} \mathrm{E}$, alt. 2425 $\mathrm{m}$, leg. PG, 28.08.2014 (POZNB 1874); W slope of Mt Končistá, $49.15735^{\circ} \mathrm{N}, 20.11323^{\circ} \mathrm{E}$, alt. $2485 \mathrm{~m}$, leg. PG, 28.08.2014 (POZNB 1917); 34UDV3645: Batizovská dolina valley, bottom plateau $\mathrm{S}$ from $\mathrm{Mt}$ Kostolík, alt. 2055 m, leg. PG, 1.08.2010 (POZNB 224); below Mt Gerlachovská veža, $49.16317^{\circ} \mathrm{N}$, $20.13428^{\circ} \mathrm{E}$, alt. $2600 \mathrm{~m}$, leg. PG, 7.08.2013 (POZNB 1631); 34UDV3747: Svištová (Bielovodská) dolina valley, Zmarznutý kotol basin, Salicetum herbaceae, alt. 2075 m, leg. PG, 1.08.2006 (POZNB 308); Svištová (Bielovodská) dolina valley, Zmarznutý kotol basin, near Zmarznuté pleso lake, $49.17645^{\circ} \mathrm{N}, 20.13918^{\circ} \mathrm{E}$, alt. $2040 \mathrm{~m}$, leg. PG, 26.09.2011 (POZNB 483); 34UDV3848: Rovienky, Zadné Rovienky, above Rovienkový vodopad waterfall, blocks of rock, $49.18848^{\circ} \mathrm{N}, 20.1513^{\circ} \mathrm{E}$, alt. $1875 \mathrm{~m}$, leg. $\mathrm{PG}$, 25.09.2011 (POZNB 494); 34UDV3849: lower, W part of Zadná Javorová dolina valley, alpine grassland near route, $49.19725^{\circ} \mathrm{N}, 20.15535^{\circ} \mathrm{E}$, alt. $1620 \mathrm{~m}$, leg. PG, 17.11.2012 (POZNB 1393); 34UDV3948: Zadná Javorová dolina valley, glacial cirque below Mt Ostrý štít, alt. 2125 m, leg. PG, 16.08.2006 (POZNB 30/2006); 34UDV4149: Kotlina Piatich Spišských Plies valley, above $\mathrm{W}$ shore of Prostredné Spišské pleso lake, alt. 2025 m, leg. PG, 27.08.2008 (POZNB 23/2007); Kotlina Piatich Spišských Plies valley, blocks of rock E from Prostredné Spišské pleso lake, alt. $2035 \mathrm{~m}$, leg. PG, 29.08.2007 (POZNB 26/2007); 34UDV4250: Velká Zmrzlá dolina valley, Barania kotlina basin, $49.20268^{\circ} \mathrm{N}, 20.20448^{\circ} \mathrm{E}$, alt. 2085 m, leg. PG, 27.09.2011 (POZNB 542); Vel'ká Zmrzlá dolina valley, below Barania kotlina basin, $49.2039^{\circ} \mathrm{N}, 20.20773^{\circ} \mathrm{E}$, alt. $1985 \mathrm{~m}$, leg. PG, 27.09.2011 (POZNB 544); Velká Zmrzlá dolina valley, blocks of rock in lower part of the valley, $49.20437^{\circ} \mathrm{N}, 20.20968^{\circ} \mathrm{E}$, alt. $1900 \mathrm{~m}$, leg. PG, 27.09.2011 (POZNB 558); 34UDV4450: Huncovská kotlina, blocks of rock filling the valley bottom, alt. 1910 m, leg. PG, 17.07.2011 (POZNB 624).

Slovakia, Western Tatra Mountains: 34UDV0453: Mt Predný Salatín, trail slope, alt. 1700 m, leg. PG, 23.07.2006 (POZNB 344); 34UDV0751: Spálená dolina valley, rocky outcrops near route, above Rázcestie k Roháčskym Plesám, alt. 1530 m, leg. PG, 24.07.2006 (POZNB 1542); 34UDV0949: Jamnícka dolina valley, Roháčsky kotol basin, near Plačlivé pliesko lake, alt. 1780 m, leg. PG, 20.07.2006 (POZNB 97).

Poland, High Tatra Mountains: 34UDV2852: Dolina Gąsienicowa valley, on the sub-glacially eroded step of Dolinka Kozia valley, alt. 1810 m, leg. PG, 21.08.2005 (POZNB 26/2005); Dolina Gąsienicowa valley, SW and above Zmarzły Staw Gąsienicowy lake, alt. 1800 m, leg. PG, 1.08.2003 (POZNB 100/2003); 34UDV2950: Dolina Pięciu Stawów Polskich valley, below Czarna Ławka pass, alt. $1870 \mathrm{~m}$, leg. PG, 27.07.2005 (POZNB 13/2005); 34UDV3149: Dolina za Mnichem valley, peat-bog near small lake below Mt Mnich, $49.1946^{\circ} \mathrm{N}, 20.0532^{\circ} \mathrm{E}$, alt. $1855 \mathrm{~m}$, leg. PG, 28.08.2012 (POZNB 1495).

Poland, Western Tatra Mountains: 34UDV1252: Dolina Jarząbcza valley, near route to Mt Trzydniowiański Wierch, trail slope, alt. $1675 \mathrm{~m}$, leg. PG, 2003 (POZNB 291); 34UDV1551: Ornak ridge, near Siwa Przełęcz pass, alt. 1770 m, leg. PG, 24.07.2009 (POZNB 13/2009); 34UDV1749: NW slope of Mt Kamienista, rocky crevices below the summit, $49.19627^{\circ} \mathrm{N}, 19.86725^{\circ} \mathrm{E}, 49.19628^{\circ} \mathrm{N}, 19.86753^{\circ} \mathrm{E}$, alt. 2040 m, $2045 \mathrm{~m}$, leg. PG, 4.08.2012 (POZNB $1147,1171)$.

Gymnomitrion adustum Nees [= Marsupella adusta (Nees) Spruce]

Poland, High Tatra Mountains: 34UDV3049: Wrota Chałubińskiego pass, alt. 2005 m, leg. PG, 28.08.2012 (POZNB 1963).

\section{Gymnomitrion concinnatum (Lightf.) Corda}

Slovakia, High Tatra Mountains: 34UDV2448: Kôprovnica valley, upper part, rocks below Mt Krížne, $49.18565^{\circ} \mathrm{N}, 19.95745^{\circ} \mathrm{E}$, alt. $1725 \mathrm{~m}$, leg. PG, 29.08.2014 (POZNB 1835); 34UDV2750: Kobylia dolina valley, rock of blocks in SW part of the valley, alt. $1790 \mathrm{~m}$, leg. PG, 29.07.2009 (POZNB 64); 34UDV2846: Nefcerka valley, above SE shore of Nižné Terianske pleso lake, near stream, $49.16727^{\circ} \mathrm{N}$, $20.01613^{\circ} \mathrm{E}$, alt. $2035 \mathrm{~m}$, leg. PG, 2.08.2012 (POZNB 1307); 34UDV2946: Furkotská dolina valley, blocks of rock near SE shore of Vyšné Wahlenbergovo pleso lake, alt. $2130 \mathrm{~m}$, leg. PG, 8.08.2008 (POZNB 13/2008); Mlynická dolina valley, blocks of rock below rocky walls descending from Mt Furkotský štit, above Okrúhle pleso lake, alt. 2135 m, leg. PG, 10.08.2008 (POZNB 19/2008); Mlynická dolina valley, N slope of Bystrá lavká pass, rocky crevices, $49.1671^{\circ} \mathrm{N}, 20.02937^{\circ} \mathrm{E}$, alt. $2230 \mathrm{~m}, 2285 \mathrm{~m}$, leg. PG, 10.08.2008, 12.08.2011 (POZNB 663, 17/2008); upper part of Mlynická dolina valley, below rocky walls descending from Mt Furkotský štit, alt. 2245 m, leg. PG, 10.08.2008 (POZNB 338); 34UDV2947: Hlinská dolina valley, rock blocks below Výsné Kôprovské sedlo pass, alt. $1810 \mathrm{~m}$, leg. PG, 18.08.2006 (POZNB 40/2006); 34UDV3046: Mlynická dolina valley, blocks of rock above $S$ shore of Vyšné Kozie pleso lake, alt. $2110 \mathrm{~m}$, leg. PG, 11.08.2008 (POZNB 22/2008); Mlynická dolina valley, $\mathrm{N}$ from $\mathrm{N}$ shore of Nižné Kozie pleso lake, $49.16622^{\circ} \mathrm{N}, 20.0425^{\circ} \mathrm{E}$, alt. $1975 \mathrm{~m}$, leg. PG, 12.08.2011 (POZNB 655); 34UDV3048: Piargová dolinka valley, glacial cirque below Mt Čubrina, blocks of rock, $49.18633^{\circ} \mathrm{N}$, 
$20.04505^{\circ} \mathrm{E}, 49.18583^{\circ} \mathrm{N}, 20.04525^{\circ} \mathrm{E}, 49.18757^{\circ} \mathrm{N}$, $20.0453^{\circ} \mathrm{E}$, alt. $1860 \mathrm{~m}, 1865 \mathrm{~m}, 1870 \mathrm{~m}, 1875 \mathrm{~m}$, $1880 \mathrm{~m}$, leg. PG, 22.07.2008, 28.07.2009 (POZNB $45 / 2006,10 / 2008,11 / 2008,12 / 2008,18 / 2009$, 19/2009, 23/2009, 24/2009); 34UDV3148: Hincova dolina valley, rocky outcrops near NW shore of Vel'ké Hincovo pleso lake, alt. 1940 m, leg. PG, 12.08.2008 (POZNB 28/2008); 34UDV3347: Žabia (Mengusovská) dolina valley, Kotlinka pod Vahou basin, alt. 2095 m, leg. PG, 13.08.2008 (POZNB 59); 34UDV3349: Žabia Bielovodská dolina valley, blocks of rock above $S$ shore of Vyšné Žabie Bielovodské pleso lake, alt. $1730 \mathrm{~m}$, leg. PG, 15.08.2009 (POZNB 39/2009); 34UDV3445: Zlomisková dolina valley, Kotlina Ladového plesa basin, rocky outcrops above $\mathrm{S}$ shore of Ladové pleso lake, below Lúčne sedlo pass, $49.16158^{\circ} \mathrm{N}, 20.10633^{\circ} \mathrm{E}$, alt. $2005 \mathrm{~m}$, leg. PG, 5.08.2010 (POZNB 16/2010); Zlomisková dolina valley, on sub-glacially eroded step of upper glacial cirque (Kotlina Ladového plesa basin), near stream, $49.16232^{\circ} \mathrm{N}, 20.10407^{\circ} \mathrm{E}$, alt. $1925 \mathrm{~m}$, leg. PG, 9.08.2011 (POZNB 651); 34UDV3448: upper part of Žabia Bielovodská dolina valley, below rocky walls between Mlynárovo sedlo and Mt Velký Žabi štít, alt. 1885 m, 1890 m, leg. PG, 19.08.2009 (POZNB 49/2009, 51/2009); 34UDV3449: Žabia Bielovodská dolina valley, blocks of rock near SE shore of Nižné Žabie Bielovodské pleso lake, alt. 1675 m, leg. PG, 14.08.2009 (POZNB 34/2009); 34UDV3545: Batizovská dolina valley, above and NW from Batizovské pleso lake, blocks of rock in the middle part of the valley, $49.15752^{\circ} \mathrm{N}, 20.1222^{\circ} \mathrm{E}$, alt. $2030 \mathrm{~m}$, leg. PG, 1.08.2010 (POZNB 3/2010); 34UDV3546: Kačacia dolina valley, upper glacial cirque (Żelazny Ogród), blocks of rock below Východná Železná brána pass, alt. 2000 m, leg. PG, 16.09.2009 (POZNB 75/2009); 34UDV3547: Kačacia dolina valley, upper (SE) part of lower glacial cirque (Gierlachowskie Spady), alt. $1750 \mathrm{~m}, 1755 \mathrm{~m}$, leg. PG, 16.09.2009 (POZNB 76/2009, 77/2009); 34UDV3645: Batizovská dolina valley, N slope of Mt Kostolík, Polytrichetum norvegici, $49.16162^{\circ} \mathrm{N}, 20.12412^{\circ} \mathrm{E}$, alt. $2240 \mathrm{~m}$, leg. $\mathrm{PG}$, 2.08.2010 (POZNB 184); 34UDV3647: Litvorová dolina valley, above Litvorové pleso lake, alt. $1885 \mathrm{~m}$, leg. PG, 17.08.2006 (POZNB 690); Litvorová dolina valley, rocky outcrops $\mathrm{E}$ from Litvorové pleso lake, alt. $1880 \mathrm{~m}, 1940 \mathrm{~m}$, leg. PG, 17.08.2006 (POZNB 34/2006, 36/2006); 34UDV3745: Sedielko nad Kotlom pass [in the ridge of Mt Kotlový štít (Malý Gerlach)], $49.16068^{\circ} \mathrm{N}, 20.14022^{\circ} \mathrm{E}$, alt. $2405 \mathrm{~m}$, leg. PG, 7.08.2013 (POZNB 1933); 34UDV3747: Svištová (Bielovodská) dolina valley, Zmarznutý kotol basin, rocky walls below Polský hrebeň pass, $49.17487^{\circ} \mathrm{N}, 20.13838^{\circ} \mathrm{E}$, alt. $2060 \mathrm{~m}$, leg. PG, 9.08.2012 (POZNB 1155); Svištová (Bielovodská) dolina valley, Zmarznutý kotol basin, alt. $2020 \mathrm{~m}$, 2060 m, leg. PG, 1.08.2006 (POZNB 10, 27/2006); 34UDV3947: Veĺka Studená dolina valley, rocky out- crops E from Dlhé pleso lake, above stream, alt. 1875 m, leg. PG, 23.08.2007 (POZNB 8/2007); Veĺka Studená dolina valley, rocky walls near stream between Dlhé pleso and Vareškové pleso lakes, alt. $1815 \mathrm{~m}$, leg. PG, 23.08.2007 (POZNB 14/2007, 15/2007); 34UDV3948: Zadná Javorová dolina valley, glacial cirque below rocky walls between Mt Ostrý štít and Mt Javorový štít, alt. 2085 m, leg. PG, 16.08.2006 (POZNB 32/2006); 34UDV3950: upper part of Suchá (Javorová) dolina valley, alt. $1860 \mathrm{~m}$, leg. PG, 18.08.2006 (POZNB 38/2006); 34UDV4048: Veĺka Studená dolina valley, Strelecké polia, $49.18443^{\circ} \mathrm{N}$, $20.18188^{\circ} \mathrm{E}$, alt. $2020 \mathrm{~m}$, leg. PG, 11.08.2011 (POZNB 667); 34UDV4049: Zadná Javorová dolina valley, in the cirque under Mt Ostrý štít, alt. $2125 \mathrm{~m}$, leg. PG, 16.08.2006 (POZNB 30/2006); 34UDV4149: Kotlina Piatich Spišských Plies valley, blocks of rock E from Prostredné Spišské pleso lake, alt. 2035 m, leg. PG, 29.08.2007 (POZNB 25/2007); 34UDV4150: Čierna Javorová dolina valley, Czarny Bandzioch, near Čierny potok stream, $49.20842^{\circ} \mathrm{N}, 20.19167^{\circ} \mathrm{E}$, alt. 1995 m, leg. PG, 26.08.2010 (POZNB 29/2010); 34UDV4250: Velká Zmrzlá dolina valley, blocks of rock above sub-glacially eroded step, $49.20448^{\circ} \mathrm{N}$, 20.20943ํㅡ, alt. $1910 \mathrm{~m}$, leg. PG, 27.09.2011 (POZNB 553); 34UDV4251: Červena dolina valley, blocks of rock near Belasé pleso lake, alt. $1865 \mathrm{~m}$, leg. PG, 25.09.2007 (POZNB 31/2007); 34UDV4450: E slope of Mt Huncowský štít, above the route Skalnaté pleso lake - Mt Veĺká Svištovka, alt. 1895 m, leg. PG, 17.07.2011 (POZNB 628); Huncovská kotlina valley, blocks of rock filling the valley bottom, alt. $1910 \mathrm{~m}$, leg. PG, 17.07.2011 (POZNB 623).

Slovakia, Western Tatra Mountains: 34UDV0453: Roháčska dolina valley, glacial cirque below $\mathrm{Mt}$ Brestová (from the north side), alt. $1720 \mathrm{~m}$, leg. PG, 23.07.2006 (POZNB 16/2006); 34UDV0551: Jalovecká dolina valley, E part of Hlboká dolina valley (Vrece), below rocky walls descending from Mt Pachol'a, alt. 1740 m, leg. PG, 19.07.2008 (POZNB 22); 34UDV1349: Račkova dolina valley, Račkov Zadok, blocks of rock SE from Vyšné Račkove pleso lake, $49.19473^{\circ} \mathrm{N}, 19.80992^{\circ} \mathrm{E}$, alt. $1675 \mathrm{~m}$, leg. PG, 21.07.2009 (POZNB 104); 34UDV1648: Kobyla ridge (SE from Mt Bystrá), rocky outcrops near route to Bystrá dolina valley, $49.18053^{\circ} \mathrm{N}, 19.84937^{\circ} \mathrm{E}$, $49.18118^{\circ} \mathrm{N}, 19.84977^{\circ} \mathrm{E}$, alt. $1955 \mathrm{~m}, 1990 \mathrm{~m}$, leg. PG, 26.08.2011 (POZNB 452).

Poland, High Tatra Mountains: 34UDV2752: Dolina Gąsienicowa valley, glacial cirque below Mt Świnica, above Czerwone Stawki lakes, Grzędy, below rocky walls descending from Mt Skrajna Turnia, alt. 1870 m, leg. PG, 20.08.2005 (POZNB 19/2005); 34UDV2852: Dolina Gąsienicowa valley, above Zmarzły Staw Gąsienicowy lake, blocks of rock, alt. $1800 \mathrm{~m}, 1845 \mathrm{~m}$, leg. PG, 2.08.2003, 21.08.2005 (POZNB 302, 931, 112/2003, 29/2005); Dolina Gąsienicowa valley, at the mouth of Zawratowy 
Żleb gully, alt. 1955 m, leg. PG, 1.08.2003 (POZNB 106/2003); 34UDV2853: Dolina Gąsienicowa valley, glacial cirque below Mt Świnica, blocks of rock near Długi Staw Gąsienicowy, alt. 1845 m, leg. PG, 20.08.2005 (POZNB 21/2005); 34UDV2950: Dolina Pięciu Stawów Polskich valley, below Czarna Ławka pass, alt. $1870 \mathrm{~m}$, leg. PG, 27.07.2005 (POZNB 13/2005, 14/2005); 34UDV2953: Dolina Pańszczycy valley, below rocky walls descending from Pańszczycka Przełęcz pass, alt. $1985 \mathrm{~m}$, leg. PG, 3.08.2003 (POZNB 114/2003, 115/2003); 34UDV3049: Dolina za Mnichem valley, below rocky walls descending from Wrota Chałubińskiego pass, alt. 1850 m, leg. PG, 16.08.2004 (POZNB 18/2004); Dolina za Mnichem valley, below Mt Cubryna, blocks of rock, alt. $2000 \mathrm{~m}$, leg. PG, 16.08.2004 (POZNB 20/2004, 22/2004); Dolina za Mnichem valley, below rocky walls descending from Mt Zadni Mnich, alt. 1980 m, leg. PG, 17.08.2004 (POZNB 23/2004); Dolina za Mnichem, rocky rubble filling the valley bottom, alt. $1790 \mathrm{~m}$, leg. PG, 27.09.2007 (POZNB 37/2007); 34UDV3050: Dolina Pięciu Stawów Polskich valley, Brzuchaty Piarg scree, below rocky walls descending from Mt Miedziane, alt. 1905 m, leg. PG, 25.07.2005 (POZNB 5/2005); 34UDV3150: Dolina Pięciu Stawów Polskich valley, rocky walls descending from Marchwiczna Przełęcz pass, alt. 1950 m, leg. PG, 28.07.2005 (POZNB 722); 34UDV3248: below Mt Kazalnica, rocks near route to Mięguszowiecka Przełęcz pod Chłopkiem pass, $49.18655^{\circ} \mathrm{N}$, 20.06722 ${ }^{\circ}$ E, alt. $1955 \mathrm{~m}$, leg. PG, 2.09.2014 (POZNB 1916); Dolina Rybiego Potoku valley, rocky outcrops above Czarny Staw pod Rysami lake, alt. 1715 m, leg. PG, 18.08.2004 (POZNB 24/2004).

Poland, Western Tatra Mountains: 34UDV1251: Dolina Jarząbcza valley, NW slope of Mt Kończysty Wierch (Jarząbczy Kopieniec), leg. PG, 28.09.2003 (POZNB 148); 34UDV1749: NW slope of Mt Kamienista, rocky crevices below the summit, $49.19628^{\circ} \mathrm{N}$, $19.86753^{\circ} \mathrm{E}$, alt. $2045 \mathrm{~m}$, leg. PG, 4.08.2012 (POZNB 1147).

Gymnomitrion brevissimum (Dumort.) Warnst. [= Marsupella brevissima (Dumort.) Grolle]

Slovakia, High Tatra Mountains: 34UDV2746: Nefcerka valley, S from S shore of Nižné Terianske pleso lake, $49.16922^{\circ} \mathrm{N}, 20.0117^{\circ} \mathrm{E}$, alt. $1940 \mathrm{~m}$, leg. $\mathrm{PG}$, 2.08.2011 (POZNB 583); Nefcerka valley, near W shore of Nižné Terianske pleso lake, alt. $1940 \mathrm{~m}$, leg. PG, 2.08.2011 (POZNB 639); 34UDV2750: Zavory pass, alt. $1860 \mathrm{~m}$, leg. PG, 29.07.2009 (POZNB 84); Zadna Tichá dolina valley, below Hladké sedlo pass, alt. 1845 m, leg. PG, 29.07.2009 (POZNB 309); 34UDV2845: Furkotská dolina valley, above N shore of Nižné Wahlenbergovo pleso lake, $49.16032^{\circ} \mathrm{N}$, $20.02475^{\circ} \mathrm{E}, 49.16042^{\circ} \mathrm{N}, 20.02452^{\circ} \mathrm{E}$, alt. $2065 \mathrm{~m}$, leg. PG, 12.08.2010 (POZNB 22/2010, 23/2010); 34UDV2846: Nefcerka valley, S from Vyšné Terian- ske pleso lake, $49.16707^{\circ} \mathrm{N}, 20.01963^{\circ} \mathrm{E}$, alt. 2125 $\mathrm{m}$, leg. PG, 2.08.2012 (POZNB 1323); 34UDV2945: Mlynická dolina valley, blocks of rock below Mt Malé Solisko, alt. 2030 m, leg. PG, 15.08.2008 (POZNB 34/2008); 34UDV2946: Furkotská dolina valley, near Vyšné Wahlenbergovo pleso lake, alt. $2100 \mathrm{~m}$, leg. PG, 8.08.2008 (POZNB 90); Furkotská dolina valley, blocks of rock near SE shore of Vyšné Wahlenbergovo pleso lake, alt. 2100 m, leg. PG, 8.08.2008 (POZNB 16/2008); Mlynická dolina valley, N slope of Bystrá lávka pass, alt. 2250 m, leg. PG, 10.08.2008 (POZNB 18/2008); 34UDV3046: Mlynická dolina valley, S from Vyšné Kozie pleso lake, Luzuletum alpino-pilosae, $49.1669^{\circ} \mathrm{N}, 20.04618^{\circ} \mathrm{E}$, alt. $2055 \mathrm{~m}$, leg. PG, 12.08.2011 (POZNB 664); 34UDV3047: Hlinská dolina valley, below Výsné Kôprovské sedlo pass, alt. $1875 \mathrm{~m}, 1950 \mathrm{~m}$, leg. PG, 21.08.2006 (POZNB 80, 119); 34UDV3148: Hincova dolina valley, rocky outcrops near $\mathrm{N}$ shore of Velké Hincovo pleso lake, alt. $1940 \mathrm{~m}$, leg. PG, 12.08.2008 (POZNB 29/2008); 34UDV3346: Drača dolinka valley, above Drače pleso lake, blocks of rock in the upper glacial cirque of the valley, $49.16768^{\circ} \mathrm{N}, 20.089^{\circ} \mathrm{E}$, alt. 2050 $\mathrm{m}$, leg. PG, 5.08.2010 (POZNB 196); 34UDV3347: Žabia Mengusovská dolina valley, Kotlinka pod Vahou basin, near hut, alt. 2150 m, leg. PG, 13.08.2008 (POZNB 91); 34UDV3349: Žabia Bielovodská dolina valley, snowbed near SE shore of Vyšné Žabie Bielovodské pleso lake, alt. $1700 \mathrm{~m}$, leg. PG, 15.08.2009 (POZNB 56); 34UDV3444: Štôlska dolina valley, upper part of the valley, below Lúčne sedlo pass, $49.15305^{\circ} \mathrm{N}, 20.10683^{\circ} \mathrm{E}$, alt. $2045 \mathrm{~m}$, leg. PG, 10.08.2010 (POZNB 20/2010); 34UDV3446: Rumanova dolinka valley, bottom plateau of lower part of the valley, $49.17002^{\circ} \mathrm{N}, 20.10235^{\circ} \mathrm{E}$, alt. 2165 m, leg. PG, 3.08.2010 (POZNB 218); Zlomisková dolina valley, Kotlina Ladového plesa basin, near $\mathrm{W}$ shore of Ladové pleso lake, $49.16352^{\circ} \mathrm{N}$, 20.1049 ${ }^{\circ}$ E, alt. $1930 \mathrm{~m}$, leg. PG, 6.08.2010 (POZNB 225); Rumanova dolinka valley, near NE shore of Rumanovo pleso lake, $49.16842^{\circ} \mathrm{N}, 20.0997^{\circ} \mathrm{E}$, alt. 2150 m, leg. PG, 3.08.2010 (POZNB 226); Rumanova dolinka valley, near NE shore of Vyšné Rumanovo pliesko lake, $49.17202^{\circ} \mathrm{N}, 20.10063^{\circ} \mathrm{E}$, alt. 2125 m, leg. PG, 4.08.2010 (POZNB 10/2010); Rumanova dolinka valley, near N shore of Vyšné Rumanovo pliesko lake, $49.17202^{\circ} \mathrm{N}, 20.1006^{\circ} \mathrm{E}, 49.17202^{\circ} \mathrm{N}$, $20.1006^{\circ} \mathrm{E}$, alt. $2125 \mathrm{~m}$, leg. PG, 4.08.2010 (POZNB 11/2010, 12/2010); 34UDV3645: Batizovská dolina valley, $\mathrm{N}$ slope of Mt Kostolík, $49.16162^{\circ} \mathrm{N}$, $20.12412^{\circ} \mathrm{E}$, alt. $2240 \mathrm{~m}$, leg. PG, 2.08.2010 (POZNB 184); below Mt Gerlachovská veža, $49.16317^{\circ} \mathrm{N}$, 20.13428 ${ }^{\circ}$ E, alt. $2590 \mathrm{~m}$, leg. PG, 7.08.2013 (POZNB 1629); 34UDV3747: Svištová (Bielovodská) dolina valley, Zmarznutý kotol basin, alt. $2005 \mathrm{~m}$, leg. PG, 1.08.2006 (POZNB 26/2006); Svištová dolina (Bielovodská) valley, upper part of the valley, $49.1802^{\circ} \mathrm{N}$, 20.13787E, alt. $1990 \mathrm{~m}$, leg. PG, 26.09.2011 
(POZNB 476); Svištová dolina (Bielovodská) valley, upper part of the valley, below Zmarznutý kotol basin, $49.18003^{\circ} \mathrm{N}, 20.1382^{\circ} \mathrm{E}$, alt. $1995 \mathrm{~m}$, leg. PG, 26.09.2011 (POZNB 477); 34UDV3846: Velická dolina valley, Vyšná Kvetnica, alt. 1885 m, leg. PG, 1.08.2006 (POZNB 113, 23/2006); 34UDV3847: Veĺka Studená dolina valley, Kotlina pod Prielomom basin, alt. $2035 \mathrm{~m}$, leg. PG, 24.08.2007 (POZNB 16/2007); 34UDV3848: Rovienky valley, Zadné Rovienky, above Rovienkový vodopad waterfall, blocks of rock, $49.18848^{\circ} \mathrm{N}, 20.1513^{\circ} \mathrm{E}$, alt. $1875 \mathrm{~m}$, leg. PG, 25.09.2011 (POZNB 494); Rovienky valley, Zadné Rovienky, $49.18767^{\circ} \mathrm{N}, 20.15355^{\circ} \mathrm{E}$, alt. 1930 $\mathrm{m}$, leg. PG, 25.09.2011 (POZNB 500); 34UDV3949: Zadná Javorová dolina valley, Sobkova úboč, blocks of rock, alt. $1985 \mathrm{~m}$, leg. PG, 21.08.2007 (POZNB 94); 34UDV4049: Zadná Javorová dolina valley, below Sedielko pass, alt. 2100 m, 2110 m, leg. PG, 16.08.2006 (POZNB 78, 92); 34UDV4148: Kotlina Piatich Spišských Plies valley, near Prostredné Spišské pleso lake, alt. 2015 m, leg. PG, 29.08.2007 (POZNB 42); 34UDV4150: Čierna Javorová dolina valley, below the sub-glacially eroded step of Barania dolinka valley (Bandzioch Czarny), $49.20817^{\circ} \mathrm{N}$, 20.19247 E, alt. $2010 \mathrm{~m}$, leg. PG, 26.08.2010 (POZNB 172); 34UDV4250: Velká Zmrzlá dolina valley, blocks of rock above sub-glacially eroded step of the valley, $49.20442^{\circ} \mathrm{N}, 20.20898^{\circ} \mathrm{E}$, alt. $1940 \mathrm{~m}$, leg. PG, 27.09.2011 (POZNB 545).

Slovakia, Western Tatra Mountains: 34UDV0452: Mt Salatín, alt. 2015 m, leg. PG, 19.07.2008 (POZNB 273); 34UDV0550: Spálená dolina valley, below Baníkovské sedlo pass, alt. $1830 \mathrm{~m}$, leg. PG, 19.07.2006 (POZNB 39); 34UDV0848: between Mt Smrek and Ziarskie sedlo pass, snowbed on main ridge, alt. $2000 \mathrm{~m}$, leg. PG, 21.07.2006 (POZNB 74); 34UDV0849: Žiarska dolina valley, Malé Závraty, below Žiarskie sedlo pass, alt. 1845 m, leg. PG, 20.07.2006 (POZNB 6/2006, 9/2006); Žiarska dolina valley, Malé Závraty, below Žiarskie sedlo pass, in the cirque below Mt Plačlivý Roháč, alt. $1835 \mathrm{~m}$, leg. PG, 20.07.2006 (POZNB 7/2006); 34UDV1250: Račkova dolina valley, Račkov Zadok, in the cirque below Mt Jakubiná, alt. 1810 m, 1830 m, leg. PG, 10.08.2004, 21.07.2009 (POZNB 4/2004, 5/2004, 6/2004, 1/2009); 34UDV1450: Gáborovo sedlo pass, alt. 1900 m, leg. PG, 24.07.2009 (POZNB 102); 34UDV1548: Bystrá dolina valley, bottom plateau of the valley, S from Dlhé Bystré pleso lake, alt. 1875 $\mathrm{m}$, leg. PG, 26.08.2011 (POZNB 459); 34UDV1649: Kamenistá dolina valley, in the cirque below $\mathrm{Mt}$ Blyšt, close to Pyšne sedlo pass, alt. 1875 m, leg. PG, 29.08.2008 (POZNB 38/2008).

Poland, High Tatra Mountains: 34UDV2752: Dolina Gąsienicowa valley, Zadnie Koło, glacial cirque below Mt Świnica, alt. 1945 m, leg. PG, 20.08.2005 (POZNB 62); 34UDV2851: Dolinka Pusta valley, snowbed near route, $49.21655^{\circ} \mathrm{N}, 20.02452^{\circ} \mathrm{E}$, alt. $1995 \mathrm{~m}$, leg. PG, 24.08.2012 (POZNB 1981); Gładka Przełęcz pass, alt. $1990 \mathrm{~m}$, leg. PG, 29.07.2009 (POZNB 108); Dolina Pięciu Stawów Polskich valley, W part of the valley, bottom plateau below Gładka Przełęcz pass, alt. $1850 \mathrm{~m}$, leg. PG, 27.07.2005 (POZNB 567, 17/2005); 34UDV2852: Dolina Gąsienicowa valley, Zawratowy Żleb gully, at the mouth of the gully, alt. 1950 m, leg. PG, 1.08.2003 (POZNB 105/2003); 34UDV2950: Dolina Pięciu Stawów Polskich valley, below Czarna Ławka ridge, alt. $1870 \mathrm{~m}$, leg. PG, 27.07.2005 (POZNB 12/2005); 34UDV2952: Dolina Gąsienicowa valley, bottom plateau of Dolinka Kozia valley, alt. 1920 m, leg. PG, 21.08.2005 (POZNB 27/2005); Dolina Gąsienicowa valley, Dolinka Kozia valley, below rocky walls of main ridge (Orla Perć), alt. 2055 m, leg. PG, 2.08.2003 (POZNB 108/2003); Dolina Gąsienicowa valley, Dolinka Kozia valley, blocks of rock, $49.22092^{\circ} \mathrm{N}, 20.02718^{\circ} \mathrm{E}$, alt. $1965 \mathrm{~m}$, leg. PG, 31.08.2011 (POZNB 656); 34UDV3049: Dolina za Mnichem valley, bottom plateau of the valley, alt. $1820 \mathrm{~m}$, leg. PG, 16.08.2004 (POZNB 15/2004); Dolina za Mnichem valley, below rocky walls descending from Mt Cubryna, alt. 2000 m, leg. PG, 16.08.2004 (POZNB 22/2004); 34UDV3050: Dolina Pięciu Stawów Polskich valley, Czerwony Piarg scree, snowbed near route below Szpiglasowa Przełęcz pass, alt. 1955 m, leg. PG, 25.07.2005 (POZNB 4/2005); 34UDV3051: Dolina Pięciu Stawów Polskich valley, S from Przedni Staw Polski lake, alt. 1670 m, leg. PG, 25.07.2005 (POZNB 6/2005); Dolina Pięciu Stawów Polskich valley, snowbed near S shore of Przedni Staw Polski lake, alt. $1665 \mathrm{~m}$, leg. PG, 26.07.2005 (POZNB 568); 34UDV3153: Dolina Waksmundzka valley, snowbed in the upper part of the valley, alt. $1950 \mathrm{~m}$, leg. PG, 22.08.2005 (POZNB 566).

\section{Lophozia wenzelii (Nees) Steph.}

Slovakia, High Tatra Mountains: 34UDV2846: Nefcerka valley, the highest glacial cirque, below Furkotské sedlo pass, $49.16905^{\circ} \mathrm{N}, 20.02283^{\circ} \mathrm{E}$, alt. 2150 $\mathrm{m}$, leg. PG, 3.08.2011 (POZNB 1982); 34UDV3746: Velická dolina valley, Velická kotlina basin, blocks of rock near Vyšné Velické plieska lakes, $49.17212^{\circ} \mathrm{N}$, 20.13678 ${ }^{\circ}$, alt. $2125 \mathrm{~m}$, leg. PG, 8.08.2012 (POZNB 1961).

\section{Marsupella boeckii (Austin) Lindb. ex Kaal.}

Slovakia, High TATra Mountains: 34UDV3746: Velická dolina valley, Velická kotlina basin, blocks of rock near Vyšné Velické plieska lakes, $49.17212^{\circ} \mathrm{N}$, 20.13678 ${ }^{\circ}$, alt. $2125 \mathrm{~m}$, leg. PG, 8.08.2012 (POZNB 1958).

Poland, High Tatra Mountains: 34UDV2851: Dolina Pięciu Stawów Polskich valley, sub-glacially eroded step of Dolinka Pusta valley, blocks of rock, $49.21427^{\circ} \mathrm{N}, 20.0227^{\circ} \mathrm{E}$, alt. $1960 \mathrm{~m}$, leg. PG, 24.08.2012 (POZNB 1964); 34UDV2959: Dolina 
Pięciu Stawów Polskich valley, blocks of rock below Mt Wyżni Liptowski Kostur, $49.20075^{\circ} \mathrm{N}, 20.02813^{\circ} \mathrm{E}$, alt. 1880 m, leg. PG, 22.08.2012 (POZNB 1967).

Marsupella condensata (Angstr. ex C. Hartm.) Lindb. ex Kaal.

Poland, High Tatra Mountains: 34UDV2852: Dolina Gąsienicowa valley, lower part of Zawratowy Żleb gully, snowbed with a northern exposure, $49.2224^{\circ} \mathrm{N}$, 20.02078 ${ }^{\circ}$, alt. $1855 \mathrm{~m}$, leg. PG, 8.08.2012 (POZNB 1965); 34UDV2959: Dolina Pięciu Stawów Polskich valley, blocks of rock below Mt Wyżni Liptowski Kostur, $49.20075^{\circ} \mathrm{N}, 20.02813^{\circ} \mathrm{E}$, alt. $1880 \mathrm{~m}$, leg. PG, 22.08.2012 (POZNB 1966).

\section{Marsupella sprucei (Limpr.) Bernet}

Slovakia, High Tatra Mountains: 34UDV2745: S slope on Mt Kriván̆, below the summit, $49.16192^{\circ} \mathrm{N}$, 20.00055 ${ }^{\circ}$ E, alt. 2455 m, leg. PG, 29.09.2013 (POZNB 1962).

\section{Marsupella emarginata (Ehrh.) Dumort.}

Slovakia, High Tatra Mountains: 34UDV3546: Kačacia dolina valley, at the mouth of Kačači žlab gully, $49.17198^{\circ} \mathrm{N}, 20.118^{\circ} \mathrm{E}$, alt. $1795 \mathrm{~m}$, leg. $\mathrm{PG}$, 28.09.2013 (POZNB 1979).

\section{Moerckia blyttii (Moerch) Brockm.}

Poland, High Tatra Mountains: 34UDV2753: Dolina Gąsienicowa valley, near Czerwone Stawki lakes, $49.22743^{\circ} \mathrm{N}, 20.00405^{\circ} \mathrm{E}$, alt. $1680 \mathrm{~m}$, leg. PG, 15.08.2013 (POZNB 1956); 34UDV3051: upper part of Dolina Roztoki valley, near Siklawa waterfall and route to the Wielki Staw Polski lake, wet rocky crevices, $49.21538^{\circ} \mathrm{N}, 20.04512^{\circ} \mathrm{E}$, alt. $1555 \mathrm{~m}$, leg. PG, 12.08.2013 (POZNB 1944).

\section{Nardia scalaris Gray}

Slovakia, High Tatra Mountains: 34UDV2746: Nefcerka valley, below Nižné Terianske pleso lake, $49.16972^{\circ} \mathrm{N}, 20.00562^{\circ} \mathrm{E}$, alt. $1890 \mathrm{~m}$, leg. $\mathrm{PG}$, 2.08.2011 (POZNB 653); 34UDV2750: Zadná Tichá dolina valley, below Hladké sedlo pass, on stone in a stream, alt. $1790 \mathrm{~m}, 1855 \mathrm{~m}$, leg. PG, 29.07.2009 (POZNB 731, 1561); 34UDV2949: Temnosmrečinská dolina valley, between Nižné Temnosmrečinské pleso and Vyšné Temnosmrečinské pleso lakes, $49.19047^{\circ} \mathrm{N}, 20.03457^{\circ} \mathrm{E}$, alt. $1695 \mathrm{~m}, 1700 \mathrm{~m}$, $1705 \mathrm{~m}$, leg. PG, 22.07.2008, 30.07.2009 (POZNB 714, 6/2008, 29/2009, 32/2009); Temnosmrečinská dolina valley, near route below Nižné Temnosmrečinské pleso lake, $49.19458^{\circ} \mathrm{N}, 20.02685^{\circ} \mathrm{E}$, alt. $1670 \mathrm{~m}$, leg. PG, 22.07.2008 (POZNB 886, 5/2008); 34UDV3048: Temnosmrečinská dolina valley, on the sub-glacially eroded step of Piargová dolinka valley, $49.18793^{\circ} \mathrm{N}, 20.04403^{\circ} \mathrm{E}$, alt. 1840 $\mathrm{m}$, leg. PG, 5.08.2011 (POZNB 658); 34UDV3445: Zlomisková dolina valley, Kotlina Ladového ple- sa basin, rocky outcrops above $\mathrm{S}$ shore of Ladové pleso lake, below Lúčne sedlo pass, $49.16193^{\circ} \mathrm{N}$, $20.10615^{\circ} \mathrm{E}, 49.16208^{\circ} \mathrm{N}, 20.10652^{\circ} \mathrm{E}$, alt. $1975 \mathrm{~m}$, leg. PG, 5.08.2010 (POZNB 228, 15/2010); Zlomisková dolina valley, on the sub-glacially eroded step of the upper part of the valley (Kotlina Ladového plesa basin), $49.16232^{\circ} \mathrm{N}, 20.10407^{\circ} \mathrm{E}$, alt. $1925 \mathrm{~m}$, leg. PG, 9.08.2011 (POZNB 650); 34UDV3447: Ťažká dolina valley, upper glacial cirque, near $\mathrm{E}$ shore of Zmarzlé pleso lake, below rocky walls descending from Mt Prédná Pustá veža, alt. $1765 \mathrm{~m}$, leg. PG, 20.08.2009 (POZNB 59/2009); 34UDV3448: Ťažká dolina valley, lower glacial cirque, above SE shore of Ťažké pleso lake, alt. 1670 m, leg. PG, 24.08.2009, 26.08.2009 (POZNB 294, 60/2009); Ťažká dolina valley, upper glacial cirque, near $\mathrm{N}$ shore of Zmarzlé pleso lake, below rocky walls descending from $\mathrm{Mt}$ Prédná Pustá veža, alt. 1760 m, leg. PG, 20.08.2009 (POZNB 55/2009); upper part of Žabia Bielovodská dolina valley, below rocky walls between Mlynárovo sedlo and Mt Velký Žabi štít, alt. 1885 m, leg. PG, 19.08.2009 (POZNB 49/2009, 50/2009); 34UDV3449: Žabia Bielovodská dolina valley, blocks of rock near SE shore of Nižné Žabie Bielovodské pleso lake, alt. 1675 m, leg. PG, 14.08.2009 (POZNB 34/2009); Žabia Bielovodská dolina valley, E from S shore of Nižné Žabie Bielovodské pleso lake, below rocky walls descending from Mt Prostredný Mlynár, alt. 1735 m, leg. PG, 16.08.2009 (POZNB 41/2009); Žabia Bielovodská dolina valley, NW from Mlynárovo sedlo pass, alt. $1800 \mathrm{~m}$, leg. PG, 15.08.2009 (POZNB 36/2009); 34UDV3547: Kačacia dolina valley, at the mouth of Kačači žlab gully, alt. 1825 m, leg. PG, 22.08.2007 (POZNB 79); Kačacia dolina valley, gully descending from Mt Zlobivá, alt. 1700 m, leg. PG, 22.08.2007 (POZNB 6/2007); Kačacia dolina valley, lower glacial cirque, alt. 1645 m, leg. PG, 22.08.2007 (POZNB 7/2007); Kačacia dolina valley, upper (SE) part of lower glacial cirque (Gierlachowskie Spady), alt. 1750 m, leg. PG, 16.09.2009 (POZNB 76/2009); 34UDV3548: Tažká dolina valley, upper part of the lower glacial cirque, blocks of rock below Mt Pustá stráž, alt. $1810 \mathrm{~m}, 1825 \mathrm{~m}$, leg. PG, 26.08.2009 (POZNB 67/2009, 70/2009); 34UDV3645: Batizovská dolina valley, Vyšna Batizovská roveň, on rocks dripping with water, $49.1596^{\circ} \mathrm{N}, 20.12772^{\circ} \mathrm{E}$, alt. $2120 \mathrm{~m}$, leg. PG, 2.08.2010 (POZNB 183); 34UDV3647: Litvorová dolina valley, below rocky walls descending from Mt Litvorový štít, alt. 1835 m, leg. PG, 15.09.2009 (POZNB 72/2009); Litvorová dolina valley, rocky outcrops $\mathrm{E}$ from Litvorové pleso lake, alt. $1940 \mathrm{~m}$, leg. PG, 17.08.2006 (POZNB 34/2006); 34UDV3848: Rovienky valley, Zadné Rovienky, $\quad 49.1877^{\circ} \mathrm{N}, \quad 20.15105^{\circ} \mathrm{E}, \quad 49.18747^{\circ} \mathrm{N}$, 20.15157 ${ }^{\circ}$ E, alt. $1910 \mathrm{~m}, 1925 \mathrm{~m}$, leg. PG, 25.09.2011 (POZNB 492, 497); 34UDV3946: Veĺka Studená dolina valley, Dolinka nad Vareškovým Plesom valley, alt. 1845 m, 1870 m, leg. PG, 23.08.2007 (POZNB 
9/2007, 13/2007); 34UDV3947: Veĺka Studená dolina valley, Zbojnícky spád, $49.1785^{\circ} \mathrm{N}, 20.17407^{\circ} \mathrm{E}$, alt. $1790 \mathrm{~m}$, leg. PG, 11.08.2011 (POZNB 580); 34UDV3948: Zadná Javorová dolina valley, glacial cirque below Mt Javorový štít, above Žabie Javorové pleso lake, alt. $1970 \mathrm{~m}$, leg. PG, 16.08.2006 (POZNB 33/2006); 34UDV3950: lower part of Suchá (Javorová) dolina valley, alt. $1835 \mathrm{~m}$, leg. PG, 18.08.2006 (POZNB 39/2006); 34UDV4050: Čierna Javorová dolina valley, Čierna záhrada, rocky walls at the mouth of Ladova dolinka valley, $49.20685^{\circ} \mathrm{N}$, $20.17762^{\circ} \mathrm{E}$, alt. $1640 \mathrm{~m}, 1660 \mathrm{~m}$, leg. PG, 25.08.2010 (POZNB 168, 24/2010); 34UDV4051: Čierna Javorová dolina valley, Čierne mlaki, on rocks dripping with water, $49.20945^{\circ} \mathrm{N}, 20.1887^{\circ} \mathrm{E}$, alt. $1910 \mathrm{~m}$, leg. PG, 26.08.2010 (POZNB 171); 34UDV4152: Kolová dolina valley, near $\mathrm{N}$ shore of Kolové pleso lake, $49.22097^{\circ} \mathrm{N}, 20.19255^{\circ} \mathrm{E}$, alt. $1565 \mathrm{~m}$, leg. PG, 28.08.2010 (POZNB 251); 34UDV4251: Červena dolina valley, near Červené pleso lake, alt. $1815 \mathrm{~m}$, leg. PG, 25.09.2007 (POZNB 32/2007, 33/2007); 34UDV4252: Dolina Bielych Plies valley, alt. 1750 m, 1840 m, leg. PG, 26.09.2007 (POZNB 34/2007, 36/2007); 34UDV4449: E slope of Mt Huncowský štít, above route from Skalnaté pleso lake to Mt Veĺká Svištovka, alt. 1895 m, leg. PG, 17.07.2011 (POZNB 632); 34UDV4450: upper part of Huncovská kotlina valley, alt. $1985 \mathrm{~m}$, leg. PG, 17.07.2011 (POZNB 626).

Slovakia, Western Tatra Mountains: 34UDV0453: Roháčska dolina valley, glacial cirque below $\mathrm{Mt}$ Brestová (from the north side), alt. $1725 \mathrm{~m}$, $1740 \mathrm{~m}, 1755 \mathrm{~m}, 1780 \mathrm{~m}$, leg. PG, 23.07.2006 (POZNB 15/2006, 17/2006, 18/2006, 19/2006); 34UDV0550: Spálená dolina valley, below Mt Baníkov, alt. 1920 m, leg. PG, 19.07.2006 (POZNB 4/2006); 34UDV0551: Jalovecká dolina valley, E part of Hlboká dolina valley (Vrece), below rocky walls descending from Mt Pachol'a, alt. 1810 m, 1815 m, leg. PG, 20.07.2008 (POZNB 2/2008, 3/2008); 34UDV0751: Roháčska dolina valley, blocks of rock near Horne Roháčske pleso lake, alt. 1730 m, $1755 \mathrm{~m}$, leg. PG, 24.07.2006 (POZNB 21/2006, 22/2006); Spálená dolina valley, rocky outcrops near route, above Rázcestie k Roháčskym Plesám, alt. 1530 m, leg. PG, 24.07.2006 (POZNB 20/2006); 34UDV0950: Smutná dolina valley, below rocky walls descending from Mt Ostrý Roháč, alt. 1810 m, leg. PG, 18.07.2006 (POZNB 1/2006); Zadná Jamnícka dolina valley (Kocioł Jamnickich Stawów), near W shore of Jamnícke Pleso Vyšné lake, alt. 1720 m, 1830 m, leg. PG, 25.08.2008 (POZNB 96, 36/2008); 34UDV1349: Račkova dolina valley, near Račkove vodopády waterfalls, $49.18995^{\circ} \mathrm{N}, 19.8145^{\circ} \mathrm{E}$, alt. 1560 m, leg. PG, 6.08.2012 (POZNB 1165); Račkova dolina valley, Račkov Zadok, near Račkove vodopády waterfalls, near route, in stream, $49.1905^{\circ} \mathrm{N}$, 19.81607E, alt. $1600 \mathrm{~m}$, leg. PG, 26.08.2011 (POZNB
1100); 34UDV1648: Kobyla ridge (SE from Mt Bystrá), rocky outcrops near route to Bystrá dolina valley, $49.18053^{\circ} \mathrm{N}, 19.84937^{\circ} \mathrm{E}$, alt. $1955 \mathrm{~m}$, leg. PG, 26.08.2011 (POZNB 452).

Poland, High Tatra Mountains: 34UDV2752: Dolina Gąsienicowa valley, glacial cirque below $\mathrm{Mt}$ Świnica, above Czerwone Stawki lakes, Grzędy, below rocky walls descending from Mt Skrajna Turnia, alt. 1805 m, 1875 m, leg. PG, 20.08.2005 (POZNB 18/2005, 20/2005); Dolina Gąsienicowa valley, glacial cirque below Mt Świnica, rocky outcrops SE from Zadni Staw Gąsienicowy lake, alt. 1875 m, leg. PG, 20.08.2005 (POZNB 25/2005); 34UDV2753: Dolina Gąsienicowa valley, near Kurtkowiec lake, alt. 1685 m, leg. PG, 30.06.2010 (POZNB 192); Dolina Gąsienicowa valley, SE from Dwoisty Staw Wschodni lake, $49.23217^{\circ} \mathrm{N}, 20.00797^{\circ} \mathrm{E}$, alt. $1815 \mathrm{~m}$, leg. PG, 20.09.2010 (POZNB 233); 34UDV2850: Dolina Pięciu Stawów Polskich valley, below Kotelnica ridge, above Czarny Staw Polski lake, alt. 1835 m, 1845 m, leg. PG, 27.07.2005 (POZNB 15/2005, 16/2005); 34UDV2851: Dolina Pięciu Stawów Polskich valley, at the mouth of Dolinka Pusta valley, $49.21063^{\circ} \mathrm{N}$, $20.0247^{\circ}$ E, alt. $1805 \mathrm{~m}$, leg. PG, 24.08.2012 (POZNB 1764); 34UDV2852: Dolina Gąsienicowa valley, above and SW from Zmarzły Staw Gąsienicowy lake, alt. $1800 \mathrm{~m}, 1810 \mathrm{~m}, 1845 \mathrm{~m}$, leg. PG, 1.08.2003, 2.08.2003, 4.08.2004, 21.08.2005 (POZNB 289, 302, 981, 98/2003, 101/2003, 102/2003, 112/2003, 29/2005); Dolina Gąsienicowa valley, at the mouth of Zawratowy Żleb gully, alt. 1955 m, leg. PG, 1.08.2003 (POZNB 106/2003); Dolina Gąsienicowa valley, below rocky walls descending from Mt Zmarzłe Czuby, alt. $1930 \mathrm{~m}$, leg. PG, 4.08.2003 (POZNB 118/2003, 119/2003); 34UDV2853: Dolina Gąsienicowa valley, above NW shore of Czarny Staw Gąsienicowy lake, alt. 1680 m, leg. PG, 31.07.2003 (POZNB 95/2003); Dolina Gąsienicowa valley, above W shore of Czarny Staw Gąsienicowy lake, at the mouth of Żleb Zaruskiego gully, alt. $1720 \mathrm{~m}$, leg. PG, 31.07.2003 (POZNB 96/2003); Dolina Gąsienicowa valley, below Zmarzły Staw lake, slighty below where the paths to Dolinka Kozia valley and Zawrat pass cross, alt. $1750 \mathrm{~m}$, leg. PG, 31.07.2003 (POZNB 89/2003); Dolina Gąsienicowa valley, near E shore of Czarny Staw Gąsienicowy lake, near route to Granaty ridge, alt. $1640 \mathrm{~m}$, leg. PG, 31.07.2003 (POZNB 90/2003, 91/2003); Dolina Gąsienicowa valley, rocky rubble above Czarny Staw Gąsienicowy lake, below Mt Kościelec, alt. 1630 m, leg. PG, 31.07.2003 (POZNB 932); 34UDV2953: Dolina Pańszczycy valley, below rocky walls descending from Pańszczycka Przełęcz pass, alt. 1985 m, leg. PG, 3.08.2003 (POZNB 114/2003, 115/2003); 34UDV3049: Dolina za Mnichem valley, above Wyżni Staw Staszica lake, snowbed, alt. 1820 m, leg. PG, 16.08.2004 (POZNB 1111, 16/2004); 34UDV3051: Dolina Pięciu Stawów Polskich valley, S from Przedni Staw Polski lake, alt. 1670 m, leg. PG, 
25.07.2005 (POZNB 6/2005); 34UDV3150: Dolina Pięciu Stawów Polskich valley, below rocky walls descending from Marchwiczna Przełęcz pass, alt. 1810 $\mathrm{m}, 1815 \mathrm{~m}, 1950 \mathrm{~m}$, leg. PG, 26.07.2005 (POZNB 8/2005, 9/2005, 11/2005); Dolina Pięciu Stawów Polskich valley, Dolinka za Niedźwiedziem valley, alt. 1785 m, leg. PG, 26.07.2005 (POZNB 7/2005); 34UDV3248: Dolina Rybiego Potoku valley, rocky outcrops above Czarny Staw pod Rysami lake, alt. 1715 m, leg. PG, 18.08.2004 (POZNB 284, 24/2004); 34UDV3353: Dolina Morskiego Oka valley, near route to Morskie Oko lake, above Wodogrzmoty Mickiewicza waterfall, leg. PG, 15.09.2004 (POZNB 1078).

Poland, Western Tatra Mountains: 34UDV1051: Dolina Wyżnia Chochołowska valley, rocky walls below Mt Łopata, alt. $1715 \mathrm{~m}$, leg. PG, 12.08.2004 (POZNB 14/2004); 34UDV1150: Dolina Jarząbcza valley, below rocky walls descending from Niska Przełęcz pass, alt. 1700 m, 1730 m, 1750 m, leg. PG, 9.08.2004 (POZNB 1/2004, 2/2004, 3/2004); 34UDV1250: Dolina Jarząbcza valley, NW slope of Mt Kończysty Wierch (Jarząbczy Kopieniec), alt. 1650 m, 1720 m, 1725 m, 1755 m, leg. PG, 22.07.2009 (POZNB 4/2009, 5/2009, 6/2009, 7/2009, 8/2009, 559); 34UDV1351: Dolina Jarząbcza valley, in a gully descending from Mt Kończysty Wierch, alt. 1750 m, leg. PG, 10.08.2004 (POZNB 7/2004); 34UDV1450: Dolina Starorobociańska valley, Szeroki Zagon, alt. $1700 \mathrm{~m}, 1760 \mathrm{~m}$, leg. PG, 12.07.2003, 11.08.2004 (POZNB 83/2003, 9/2004, 12/2004, 13/2004).

Pseudolophozia sudetica (Nees ex Huebener) Konst. et Vilnet [= Lophozia sudetica (Nees ex Huebener) Grolle]

Slovakia, High Tatra Mountains: 34UDV2745: S slopes of Mt Kriván, below the summit, $49.16237^{\circ} \mathrm{N}$, 20.00027 E, alt. $2480 \mathrm{~m}$, leg. PG, 29.09.2013 (POZNB 1708); Važecká dolina valley, near SE shore of Krivánske Zelené pleso lake, blocks of rock, $49.15792^{\circ} \mathrm{N}$, 20.00877 E, alt. $2000 \mathrm{~m}$, leg. PG 11.08.2010 (POZNB 204); 34UDV2746: Nefcerka valley, near W shore of Nižné Terianske pleso lake, alt. $1940 \mathrm{~m}$, leg. PG, 2.08.2011 (POZNB 640); 34UDV2746: Nefcerka valley, blocks of rock below rocky walls descending from Mt Kriváň, $49.16785^{\circ} \mathrm{N}, 20.00272^{\circ} \mathrm{E}$, alt. 1830 $\mathrm{m}$, leg. PG, 2.08.2011 (POZNB 644); 34UDV2750: Kobylia dolina valley, rock of blocks in SW part of the valley, alt. $1790 \mathrm{~m}$, leg. PG, 29.07.2009 (POZNB 64); upper part of Zadna Tichá dolina valley, alt. $1770 \mathrm{~m}$, leg. PG, 29.07.2009 (POZNB 25/2009); 34UDV2845: Furkotská dolina valley, above $\mathrm{N}$ shore of Nižné Wahlenbergovo pleso lake, $49.16042^{\circ} \mathrm{N}, 20.02452^{\circ} \mathrm{E}$, alt. 2065 m, leg. PG, 12.08.2010 (POZNB 23/2010); Furkotská dolina valley, blocks of rock near SE shore of Vyšné Wahlenbergovo pleso lake, alt. $2130 \mathrm{~m}$, leg. PG, 8.08.2008 (POZNB 13/2008); 34UDV2846: Nefcerka valley, the highest glacial cirque, below
Furkotské sedlo pass, $49.16905^{\circ} \mathrm{N}, 20.02283^{\circ} \mathrm{E}$, alt. 2150 m, leg. PG, 3.08.2011 (POZNB 1983); Furkotská dolina valley, blocks of rock near $\mathrm{S}$ shore of Vyšné Wahlenbergovo pleso, alt. 2150 m, leg. PG, 10.08.2008 (POZNB 21/2008); Nefcerka valley, near SW shore of Vyšné Terianské pleso lake, $49.16768^{\circ} \mathrm{N}$, 20.02032 ${ }^{\circ}$ E, alt. 2085 m, leg. PG, 3.08.2011 (POZNB 606); Nefcerka valley, rock boulders $\mathrm{E}$ from $\mathrm{E}$ shore of Nižné Terianske pleso lake, snowbed, $49.16727^{\circ} \mathrm{N}$, 20.01613 ${ }^{\circ}$ E, alt. $2035 \mathrm{~m}$, leg. PG, 2.08.2012 (POZNB 1326); Nefcerka valley, blocks of rock $S$ from Vyšné Terianske pleso lake, $49.1658^{\circ} \mathrm{N}, 20.0198^{\circ} \mathrm{E}$, alt. 2125 m, leg. PG (POZNB 1968);n 34UDV2945: Mlynická dolina valley, blocks of rock below Mt Malé Solisko, alt. 2015 m, leg. PG, 15.08.2008 (POZNB 105); 34UDV2946: Furkotská dolina valley, blocks of rock near SE shore of Vyšné Wahlenbergovo pleso lake, alt. $2100 \mathrm{~m}, 2130 \mathrm{~m}$, leg. PG, 8.08.2008 (POZNB 14/2008, 16/2008); Mlynická dolina valley, blocks of rock below rocky walls descending from Mt Furkotský štit, above Okrúhle pleso lake, alt. 2135 m, leg. PG, 10.08.2008 (POZNB 19/2008); Mlynická dolina valley, $\mathrm{N}$ slope of Bystrá lávka pass, $49.1671^{\circ} \mathrm{N}$, $20.02937^{\circ} \mathrm{E}$, alt. $2230 \mathrm{~m}, 2250 \mathrm{~m}, 2285 \mathrm{~m}$, leg. PG, 10.08.2008, 12.08.2011 (POZNB 663, 17/2008, 18/2008); 34UDV2949: Temnosmrečinská dolina valley, rocky outcrops between Nižné Temnosmrečinské pleso and Vyšné Temnosmrečinské pleso lakes, $49.19047^{\circ} \mathrm{N}, 20.03457^{\circ} \mathrm{E}$, alt. $1700 \mathrm{~m}, 1705 \mathrm{~m}$, leg. PG, 22.07.2008, 30.07.2009 (POZNB 6/2008, 29/2009, 30/2009, 31/2009); Temnosmrečinská dolina valley, rocky outcrops near route, below Nižné Temnosmrečinské pleso lake, $49.19458^{\circ} \mathrm{N}$, 20.02685 ${ }^{\circ}$, alt. $1670 \mathrm{~m}$, leg. PG, 22.07.2008, 30.07.2009 (POZNB 5/2008, 26/2009, 27/2009, 28/2009); 34UDV3045: Mlynická dolina valley, above S shore of Nižne Kozie pleso lake, alt. 1945 m, leg. PG, 11.08.2008 (POZNB 26/2008); Mlynická dolina valley, below rocky walls descending from $\mathrm{Mt}$ Malé Solisko, alt. $1945 \mathrm{~m}$, leg. PG, 15.08.2008 (POZNB 33/2008); Mlynická dolina valley, near Volie pliesko lake, alt. $1915 \mathrm{~m}$, leg. PG, 11.08.2008 (POZNB 27/2008); 34UDV3046: Mlynická dolina valley, blocks of rock above $S$ shore of Vyšné Kozie pleso lake, alt. $2110 \mathrm{~m}$, leg. PG, 11.08.2008 (POZNB 22/2008); upper, NE part of Mlynická dolina valley, blocks of rock, below rocky walls descending from Mt Velka Capia veža, alt. 2100 m, leg. PG, 11.08.2008 (POZNB 24/2008, 25/2008); 34UDV3047: Hlinská dolina valley, blocks of rock below Výsné Kôprovské sedlo pass, alt. $1810 \mathrm{~m}, 1950 \mathrm{~m}$, leg. PG, 18.08.2006 (POZNB 40/2006, 41/2006, 42/2006, 43/2006); 34UDV3048: Piargová dolinka valley, glacial cirque below Mt Čubrina, blocks of rock, $49.18583^{\circ} \mathrm{N}$, $20.04525^{\circ} \mathrm{E}, 49.18633^{\circ} \mathrm{N}, 20.04505^{\circ} \mathrm{E}, 49.18657^{\circ} \mathrm{N}$, $20.04512^{\circ} \mathrm{E}, 49.18688^{\circ} \mathrm{N}, 20.04473^{\circ} \mathrm{E}, 49.18705^{\circ} \mathrm{N}$, $20.04422^{\circ} \mathrm{E}, 49.18757^{\circ} \mathrm{N}, 20.0453^{\circ} \mathrm{E}$, alt. $1860 \mathrm{~m}$, 1865 m, 1870 m, 1875 m, 1880 m, 1885 m, leg. PG, 
22.08.2006, 22.07.2008, 28.07.2009 (POZNB 20, 44/2006, 45/2006, 7/2008, 8/2008, 9/2008, $10 / 2008,11 / 2008,12 / 2008,18 / 2009,19 / 2009$, 20/2009, 21/2009, 22/2009, 23/2009, 24/2009); 34UDV3147: Hincova dolina valley, rocky outcrops near NW shore of Velké Hincovo pleso lake, alt. 1940 $\mathrm{m}$, leg. PG, 12.08.2008 (POZNB 28/2008); 34UDV3148: Hincova dolina valley, rocky outcrops near N shore of Velké Hincovo pleso lake, alt. 1940 m, leg. PG, 12.08.2008 (POZNB 29/2008, 30/2008); 34UDV3247: Žabia Mengusovská dolina valley, Kotlina Žabich plies basin, blocks of rock $\mathrm{N}$ and above Velké Žabie Pleso Mengusovské lake, alt. $1870 \mathrm{~m}$, $1935 \mathrm{~m}$, leg. PG, 13.08.2008 (POZNB 31/2008, 32/2008); 34UDV3344: Mengusovská dolina valley, near route from Popradské pleso lake to Mt Ostrva, alt. $1675 \mathrm{~m}$, leg. PG, 10.08.2010 (POZNB 211); 34UDV3346: Drača dolinka valley, blocks of rock above Drače pleso lake, $49.16772^{\circ} \mathrm{N}, 20.0865^{\circ} \mathrm{E}$, alt. 2075 m, leg. PG, 5.08.2010 (POZNB 14/2010); 34UDV3347: Žabia Mengusovská dolina valley, Kotlinka pod Vahou basin, alt. $2095 \mathrm{~m}$, leg. PG, 13.08.2008 (POZNB 59); 34UDV3349: Žabia Bielovodská dolina valley, blocks of rock above $S$ shore of Vyšné Žabie Bielovodské pleso lake, alt. $1705 \mathrm{~m}$, $1730 \mathrm{~m}$, leg. PG, 15.08.2009, 19.09.2009 (POZNB 37/2009, 38/2009, 39/2009, 79/2009); Žabia Bielovodská dolina valley, rocky walls dripping with water above SE shore of Vyšné Žabie Bielovodské pleso lake, alt. 1730 m, leg. PG, 21.09.2009 (POZNB 348); 34UDV3444: upper part of Štôlska dolina valley, below Lúčne sedlo pass, $49.15158^{\circ} \mathrm{N}, 20.1062^{\circ} \mathrm{E}$, $49.15278^{\circ} \mathrm{N}, 20.1065^{\circ} \mathrm{E}, 49.1527^{\circ} \mathrm{N}, 20.10607^{\circ} \mathrm{E}$, alt. $2000 \mathrm{~m}, 2025 \mathrm{~m}, 2055 \mathrm{~m}$, leg. PG, 10.08.2010 (POZNB 178, 234, 1505); 34UDV3445: Zlomisková dolina valley, Kotlina Ladového plesa basin, near SE shore of Ladové pleso lake, $49.16285^{\circ} \mathrm{N}, 20.10755^{\circ} \mathrm{E}$, alt. $1930 \mathrm{~m}$, leg. PG, 6.08.2010 (POZNB 19/2010); Zlomisková dolina valley, Kotlina Ladového plesa basin, rocky outcrops above $S$ shore of Ladové pleso lake, below Lúčne sedlo pass, $49.16158^{\circ} \mathrm{N}$, $20.10633^{\circ} \mathrm{E}, 49.16193^{\circ} \mathrm{N}, 20.10615^{\circ} \mathrm{E}, 49.16208^{\circ} \mathrm{N}$, $20.10652^{\circ} \mathrm{E}$, alt. $1975 \mathrm{~m}, 1980 \mathrm{~m}, 2005 \mathrm{~m}$, leg. PG, 5.08.2010 (POZNB 228, 15/2010, 16/2010); Zlomisková dolina valley, on sub-glacially eroded step upper glacial cirque (Kotlina Ladového plesa basin), near stream, $49.1624^{\circ} \mathrm{N}, 20.1029^{\circ} \mathrm{E}$, alt. $1880 \mathrm{~m}$, leg. PG, 9.08.2011 (POZNB 647); 34UDV3446: Rumanova dolinka valley, near $\mathrm{NE}$ and $\mathrm{N}$ shore of $\mathrm{Ru}-$ manovo pleso lake, $49.16842^{\circ} \mathrm{N}, 20.0997^{\circ} \mathrm{E}$, $49.17037^{\circ} \mathrm{N}, 20.10015^{\circ} \mathrm{E}$, alt. $2150 \mathrm{~m}, 2160 \mathrm{~m}$, leg. PG, 3.08.2010 (POZNB 188, 226); Rumanova dolinka valley, lower part of the valley, snowbed with Gnaphalium supinum, $49.17002^{\circ} \mathrm{N}, 20.10235^{\circ} \mathrm{E}$, alt. 2165 m, leg. PG, 3.08.2010 (POZNB 218); Rumanova dolinka valley, near $\mathrm{E}$ shore of Vyšné Rumanovo pliesko lake, $49.1719^{\circ} \mathrm{N}, 20.1008^{\circ} \mathrm{E}, 49.17202^{\circ} \mathrm{N}$, $20.1006^{\circ}$ E, alt. $2125 \mathrm{~m}$, leg. PG, 3.08.2010, 4.08.2010
(POZNB 9/2010, 11/2010); Rumanova dolinka valley, near $\mathrm{N}$ shore of Vyšné Rumanovo pliesko lake, $49.17202^{\circ} \mathrm{N}, 20.1006^{\circ} \mathrm{E}, 49.17202^{\circ} \mathrm{N}, 20.1006^{\circ} \mathrm{E}$, alt. $2125 \mathrm{~m}$, leg. PG, 4.08.2010 (POZNB 12/2010, 13/2010); Rumanova dolinka valley, near NE shore of Vyšné Rumanovo pliesko lake, $49.17202^{\circ} \mathrm{N}$, $20.10063^{\circ} \mathrm{E}$, alt. $2125 \mathrm{~m}$, leg. PG, 4.08.2010 (POZNB 10/2010); Rumanova dolinka valley, blocks of rock above Vyšné Rumanovo pliesko lake, $49.17212^{\circ} \mathrm{N}$, 20.10102 ${ }^{\circ}$ E, alt. $2135 \mathrm{~m}$, leg. PG, 9.08.2011 (POZNB 573); 34UDV3447: Ťažká dolina valley, upper glacial cirque, blocks of rock above $S$ shore of Zmarzlé pleso lake, alt. 1715 m, 1805 m, leg. PG, 20.08.2009 (POZNB 56/2009, 58/2009); Ťažká dolina valley, upper glacial cirque, blocks of rock below Mt Ganek and Mt Vysoká, alt. 1990 m, leg. PG, 20.08.2009 (POZNB 57/2009); Ťažká dolina valley, upper glacial cirque, blocks of rock below Mt Kačacia veža, alt. $1805 \mathrm{~m}, 1820 \mathrm{~m}$, leg. PG, 24.08.2009 (POZNB 61/2009, 62/2009); Ťažká dolina valley, upper glacial cirque, near E shore of Zmarzlé pleso lake, below rocky walls descending from Mt Prédná Pustá veža, alt. 1765 m, leg. PG, 20.08.2009 (POZNB 59/2009); 34UDV3448: Spádová dolinka valley, SW part of the glacial cirque, blocks of rock below rocky walls descending from Mt Malé Rysy, alt. 1995 m, 2020 m, leg. PG, 25.08.2009 (POZNB 65/2009, 66/2009); Ťažká dolina valley, lower glacial cirque, above SE shore of Ťažké pleso lake, alt. $1670 \mathrm{~m}$, leg. PG, 24.08.2009 (POZNB 60/2009); Tažká dolina valley, upper glacial cirque, near $\mathrm{N}$ shore of Zmarzlé pleso lake, below rocky walls descending from Mt Prédná Pustá veža, alt. 1760 m, leg. PG, 20.08.2009 (POZNB 55/2009); upper part of Žabia Bielovodská dolina valley, below rocky walls between Mlynárovo sedlo and Mt Velký Žabi štít, alt. 1885 m, 1890 m, 1920 m, leg. PG, 17.08.2009, 19.08.2009 (POZNB 46/2009, 47/2009, 49/2009, 50/2009, 51/2009, 52/2009, 53/2009); 34UDV3449: Žabia Bielovodská dolina valley, blocks of rock near SE shore of Nižné Žabie Bielovodské pleso lake, alt. $1675 \mathrm{~m}$, leg. PG, 14.08.2009 (POZNB 34/2009); Žabia Bielovodská dolina valley, E from S shore of Nižné Žabie Bielovodské pleso lake, below rocky walls descending from Mt Prostredný Mlynár, alt. 1735 m, leg. PG, 16.08.2009 (POZNB 40/2009, 41/2009); Žabia Bielovodská dolina valley, NW from Mlynárovo sedlo pass, alt. $1800 \mathrm{~m}, 1920 \mathrm{~m}$, leg. PG, 15.08.2009, 16.08.2009 (POZNB 36/2009, 43/2009, 44/2009, 45/2009); Žabia Bielovodská dolina valley, rocky walls between Mlynárovo sedlo pas and Mt Velký Žabi štít, alt. 1920 m, leg. PG, 18.08.2009 (POZNB 48/2009); 34UDV3545: Batizovská dolina valley, blocks of rock in the middle part of the valley, above Batizovské pleso lake, $49.15752^{\circ} \mathrm{N}, 20.1222^{\circ} \mathrm{E}$, alt. 2030 m, leg. PG, 1.08.2010 (POZNB 3/2010); Batizovská dolina valley, blocks of rock near $\mathrm{S}$ shore of Pliesko pod Kostolikom lake, $49.1603^{\circ} \mathrm{N}, 20.12135^{\circ} \mathrm{E}$, 
alt. 2045 m, leg. PG, 1.08.2010 (POZNB 2/2010); Batizovská dolina valley, blocks of rock near SE shore of Pliesko pod Kostolikom lake, $49.16117^{\circ} \mathrm{N}$, 20.12187 E, alt. $2055 \mathrm{~m}$, leg. PG, 1.08.2010 (POZNB $1 / 2010)$; S slope of Mt Končistá, $49.15527^{\circ} \mathrm{N}$, $20.11467^{\circ} \mathrm{E}, 49.15617^{\circ} \mathrm{N}, 20.11413^{\circ} \mathrm{E}$, alt. $2425 \mathrm{~m}$, 2475 m, leg. PG, 28.08.2014 (POZNB 1872, 1874); Zlomisková dolina valley, Kotlina Lodového plesa basin, rocky walls above $\mathrm{E}$ shore of Lodové pleso lake, $49.16292^{\circ} \mathrm{N}, 20.10985^{\circ} \mathrm{E}, 49.16292^{\circ} \mathrm{N}, 20.10985^{\circ} \mathrm{E}$, alt. 2010 m, leg. PG, 6.08.2010 (POZNB 17/2010, 18/2010); 34UDV3546: Kačacia dolina valley, upper glacial cirque (Żelazny Ogród), blocks of rock below Východná Železná brána pass, alt. 1985 m, 2000 m, leg. PG, 16.09.2009 (POZNB 143, 74/2009, 75/2009); Zlomisková dolina valley, Železná kotlina basin (below Východná Železná braná pass), $49.16393^{\circ} \mathrm{N}$, 20.11097E, alt. $2080 \mathrm{~m}$, leg. PG, 6.08.2010 (POZNB 214); 34UDV3547: Kačacia dolina valley, gully descending from Mt Zlobivá, alt. 1700 m, leg. P. Górski, 22.08.2007 (POZNB 6/2007); Kačacia dolina valley, lower glacial cirque, alt. 1645 m, leg. PG, 22.08.2007 (POZNB 7/2007); Kačacia dolina valley, rocky walls below Mt Ganek, alt. 1665 m, leg. PG, 22.08.2007 (POZNB 3/2007); Kačacia dolina valley, upper (SE) part of lower glacial cirque (Gierlachowskie Spady), alt. 1750 m, leg. PG, 16.09.2009 (POZNB 76/2009); 34UDV3548: Tažká dolina valley, upper part of the lower glacial cirque, blocks of rock below Mt Pustá stráž, alt. $1810 \mathrm{~m}, 1815 \mathrm{~m}, 1825 \mathrm{~m}$, leg. PG, 24.08.2009, 26.08.2009 (POZNB 974, 63/2009, 64/2009, 67/2009, 68/2009, 71/2009);34UDV3645: Batizovská dolina valley, Vyšna Batizovská roveň, Batizovské oká lakes, $49.16318^{\circ} \mathrm{N}, 20.12695^{\circ} \mathrm{E}$, alt. $2250 \mathrm{~m}$, leg. PG, 2.08.2010 (POZNB 8/2010); 34UDV3647: Litvorová dolina valley, below rocky walls descending from Mt Litvorový štít, alt. $1835 \mathrm{~m}$, leg. PG, 15.09.2009 (POZNB 72/2009); Litvorová dolina valley, glacial cirque below Mt Litvorový štít, alt. 1860 m, leg. PG, 17.08.2006 (POZNB 37/2006); Litvorová dolina valley, rocky outcrops $\mathrm{E}$ from Litvorové pleso lake, alt. $1880 \mathrm{~m}, 1940 \mathrm{~m}, 1990 \mathrm{~m}$, leg. PG, 17.08.2006 (POZNB 34/2006, 35/2006, 36/2006); Svištová (Bielovodská) dolina valley, Vyšna Svištová roveň, $49.18063^{\circ} \mathrm{N}, 20.13553^{\circ} \mathrm{E}$, alt. $1985 \mathrm{~m}$, leg. PG, 26.09.2011 (POZNB 474); 34UDV3745: Sedielko nad Kotlom pass [in the ridge of Mt Kotlový štít (Malý Gerlach) ], $49.16068^{\circ} \mathrm{N}, 20.14022^{\circ} \mathrm{E}$, alt. 2405 m, leg. PG, 7.08.2013 (POZNB 1933); 34UDV3746: Velická dolina valley, Velická kotlina basin, blocks of rock near Vyšné Velické plieska lakes, $49.1723^{\circ} \mathrm{N}$, $20.13588^{\circ} \mathrm{E}$, alt. $2125 \mathrm{~m}$, leg. PG, 8.08.2012 (POZNB 1145); 34UDV3747: Svištová (Bielovodská) dolina valley, Zmarznutý kotol basin, $49.17898^{\circ} \mathrm{N}$, $20.13897^{\circ}$ E, alt. 2015 m, 2035 m, 2060 m, 2065 m, leg. PG, 1.08.2006, 26.09.2011 (POZNB 480, 24/2006, 25/2006, 27/2006); Svištová (Bielovodská) dolina valley, Zmarznutý kotol basin, near Zmarznuté pleso lake, $49.17645^{\circ} \mathrm{N}, 20.13918^{\circ} \mathrm{E}$, alt. $2040 \mathrm{~m}$, leg. PG, 26.09.2011 (POZNB 481); Svištová (Bielovodská) dolina valley, blocks of rock below Zmarznutý kotol basin, $49.17915^{\circ} \mathrm{N}, 20.13968^{\circ} \mathrm{E}$, alt. $2025 \mathrm{~m}$, leg. PG, 26.09.2011 (POZNB 486); 34UDV3847: Veĺka Studená dolina valley, Kotlina pod Prielomom basin, alt. $2035 \mathrm{~m}$, leg. PG, 24.08.2007 (POZNB 16/2007); 34UDV3848: Rovienky valley, Zadné Rovienky, $49.18747^{\circ} \mathrm{N}, \quad 20.15157^{\circ} \mathrm{E}, \quad 49.18767^{\circ} \mathrm{N}$, $20.15355^{\circ} \mathrm{E}, 49.18745^{\circ} \mathrm{N}, 20.15342^{\circ} \mathrm{E}$, alt. $1925 \mathrm{~m}$, 1930 m, 1950 m, leg. PG, 25.09.2011 (POZNB 497, 499, 604); Rovienky, Zadné Rovienky, above Rovienkový vodopad waterfall, blocks of rock, $49.18848^{\circ} \mathrm{N}$, $20.1513^{\circ} \mathrm{E}, 49.1877^{\circ} \mathrm{N}, 20.15105^{\circ} \mathrm{E}$, alt. $1875 \mathrm{~m}$, 1910 m, leg. PG, 25.09.2011 (POZNB 492, 494); 34UDV3946: Veĺka Studená dolina valley, Dolinka nad Vareškovým Plesom valley, alt. 1845 m, 1850 m, $1870 \mathrm{~m}$, leg. PG, 23.08.2007 (POZNB 9/2007, 10/2007, 13/2007); 34UDV3947: Veĺka Studená dolina valley, blocks of rock between Dlhé pleso lake and Zbojnícka chata hut, alt. $1900 \mathrm{~m}$, leg. PG, 24.08.2007 (POZNB 17/2007); Veĺka Studená dolina valley, blocks of rock $\mathrm{W}$ from Zbojnícka chata hut, snowbed among blocks of rock, alt. $1935 \mathrm{~m}$, leg. PG, 24.08.2007 (POZNB 8); Veĺka Studená dolina valley, rocky outcrops $\mathrm{E}$ from Dlhé pleso lake, above stream, alt. 1875 m, leg. PG, 23.08.2007 (POZNB 8/2007); Veĺka Studená dolina valley, rocky walls near stream between Dlhé pleso and Vareškové pleso lake, alt. 1815 m, leg. PG, 23.08.2007 (POZNB 14/2007, 15/2007); Veĺka Studená dolina valley, Zbojnícky spád, $49.1785^{\circ} \mathrm{N}, 20.17407^{\circ} \mathrm{E}$, alt. $1790 \mathrm{~m}$, leg. $\mathrm{PG}$, 11.08.2011 (POZNB 580); 34UDV3948: Zadná Javorová dolina valley, glacial cirque below Mt Javorový štít, above Žabie Javorové pleso lake, alt. 1970 m, leg. PG, 16.08.2006 (POZNB 33/2006); Zadná Javorová dolina valley, glacial cirque below Mt Ostrý štít, alt. $2210 \mathrm{~m}, 2125 \mathrm{~m}$, leg. PG, 16.08.2006 (POZNB 29/2006, 31/2006, 30/2006); Zadná Javorová dolina valley, glacial cirque below rocky walls between Mt Ostrý štít and Mt Javorový štít, alt. 2085 $\mathrm{m}$, leg. PG, 16.08.2006 (POZNB 32/2006); 34UDV3950: Suchá (Javorová) dolina valley, alt. $1835 \mathrm{~m}, 1860 \mathrm{~m}$, leg. PG, 18.08.2006 (POZNB 38/2006, 39/2006); 34UDV4047: Veĺka Studená dolina valley, Dolinka nad Vareškovým Plesom valley, alt. 1860 m, leg. PG, 23.08.2007 (POZNB 12/2007); 34UDV4048: Veĺka Studená dolina valley, Strelecké polia, $49.18543^{\circ} \mathrm{N}, 20.17883^{\circ} \mathrm{E}$, alt. $2105 \mathrm{~m}$, leg. PG, 11.08.2011 (POZNB 661); 34UDV4049: Zadná Javorová dolina valley, below rocky walls descending from Mt Ostrí štít, alt. 2140 m, leg. PG, 16.08.2006 (POZNB 38); Zadná Javorová dolina valley, below Sedielko pass, alt. $2110 \mathrm{~m}$, leg. PG, 16.08.2006 (POZNB 78); 34UDV4050: Čierna Javorová dolina valley, Čierne mlaki, blocks of rock below rocky walls descending from Hrebeň Snehových veži ridge, $49.20793^{\circ} \mathrm{N}, 20.18702^{\circ} \mathrm{E}, 49.2075^{\circ} \mathrm{N}, 20.18718^{\circ} \mathrm{E}$, 
alt. 1960 m, 1975 m, 2005 m, leg. PG, 26.08.2010 (POZNB 164, 25/2010, 26/2010); Čierna Javorová dolina valley, upper glacial cirque (Čierne mlaki), $49.2084^{\circ} \mathrm{N}, 20.18747^{\circ} \mathrm{E}$, alt. $1940 \mathrm{~m}$, leg. PG, 26.08.2010 (POZNB 163); 34UDV4051: Čierna Javorová dolina valley, Čierna záhrada, blocks of rock in the upper part of the valley, near Czarna Siklawa stream, $49.21018^{\circ} \mathrm{N}, 20.17982^{\circ} \mathrm{E}$, alt. $1635 \mathrm{~m}$, leg. PG, 25.08.2010 (POZNB 165); Čierna Javorová dolina valley, Čierne mlaki, on rocks dripping with water, $49.20945^{\circ} \mathrm{N}, 20.1887^{\circ} \mathrm{E}$, alt. $1910 \mathrm{~m}$, leg. PG, 26.08.2010 (POZNB 171); 34UDV4148: Kotlina Piatich Spišských Plies valley, near Nižne Spišské pleso lake, alt. $1990 \mathrm{~m}$, leg. PG, 29.08.2007 (POZNB 28/2007); Malá Studená dolina valley, Dolina pod Sedielkom valley, alt. 2065 m, leg. PG, 21.08.2007 (POZNB 2/2007); upper part of Malá Studená dolina valley, alt. $1935 \mathrm{~m}, 1945 \mathrm{~m}$, leg. PG, 29.08.2007 (POZNB 29/2007, 30/2007); 34UDV4149: Kotlina Piatich Spišských Plies valley, above $\mathrm{W}$ shore of Prostredné Spišské pleso lake, alt. 2000 m, 2005 m, 2010 m, 2025 m, leg. PG, 27.08.2007 (POZNB 18/2007, 19/2007, 20/2007, 21/2007, 22/2007, 23/2007, 27/2007); Kotlina Piatich Spišských Plies valley, blocks of rock E from Prostredné Spišské pleso lake, alt. 2035 m, leg. PG, 29.08.2007 (POZNB 25/2007); 34UDV4150: Čierna Javorová dolina valley, Czarny Bandzioch, near Čierny potok stream, $49.20842^{\circ} \mathrm{N}$, 20.19167 E, alt. 1995 m, leg. PG, 26.08.2010 (POZNB 29/2010); 34UDV4152: Kolová dolina valley, above and SEE from Kolové pleso lake, below rocky walls descending from Hrebeň Kolových veži ridge, $49.21843^{\circ} \mathrm{N}, 20.19438^{\circ} \mathrm{E}$, alt. $1645 \mathrm{~m}$, leg. PG, 28.08.2010 (POZNB 255); Kolová dolina valley, blocks of rock in upper part of the valley, $49.21825^{\circ} \mathrm{N}$, $20.20028^{\circ} \mathrm{E}$, alt. $1820 \mathrm{~m}$, leg. PG, 1.09.2011 (POZNB 448); 34UDV4250: upper part of Vel'ká Zmrzlá dolina valley, Barania kotlina basin, blocks of rock, $49.20367^{\circ} \mathrm{N}, 20.20733^{\circ} \mathrm{E}$, alt. $2005 \mathrm{~m}$, leg. PG, 27.09.2011 (POZNB 540); Velká Zmrzlá dolina valley, blocks of rock above the sub-glacially eroded step of the valley, $49.20422^{\circ} \mathrm{N}, 20.20942^{\circ} \mathrm{E}, 49.20433^{\circ} \mathrm{N}$, $20.20925^{\circ} \mathrm{E}, 49.20437^{\circ} \mathrm{N}, 20.20968^{\circ} \mathrm{E}, 49.20448^{\circ} \mathrm{N}$, $20.20943^{\circ} \mathrm{E}$, alt. $1900 \mathrm{~m}, 1910 \mathrm{~m}, 1925 \mathrm{~m}$, leg. PG, 27.09.2011 (POZNB 547, 549, 554, 557); 34UDV4251: Červena dolina valley, blocks of rock near Belasé pleso lake, alt. 1865 m, leg. PG, 25.09.2007 (POZNB 31/2007); Červena dolina valley, near Červené pleso lake, alt. $1815 \mathrm{~m}$, leg. PG, 25.09.2007 (POZNB 32/2007, 33/2007): 34UDV 4252: Dolina Bielych Plies valley, blocks of rock, alt. $1750 \mathrm{~m}, 1780$ $\mathrm{m}, 1840 \mathrm{~m}$, leg. PG, 26.09.2007 (POZNB 337, 34/2007, 35/2007, 36/2007); 34UDV4450: E slope of Mt Huncowský štít, above the route Skalnaté pleso lake - Mt Veĺká Svištovka, alt. 1985 m, leg. PG, 17.07.2011 (POZNB 625); Huncovská kotlina valley, blocks of rock filling the valley bottom, alt. $1910 \mathrm{~m}$, leg. PG, 17.07.2011 (POZNB 622).
Slovakia, Western Tatra Mountains:34UDV0453: Roháčska dolina valley, glacial cirque below Mt Brestová (from the north side), alt. $1720 \mathrm{~m}, 1740 \mathrm{~m}, 1755$ m, 1780 m, leg. PG, 23.07.2006 (POZNB 16/2006, 17/2006, 18/2006, 19/2006); 34UDV0550: Spálená dolina valley, below Baníkovské sedlo pass, alt. 1855 m, leg. PG, 19.07.2006 (POZNB 23); 34UDV0551: Jalovecká dolina valley, E part of Hlboká dolina valley (Vrece), below rocky walls descending from Mt Pachol'a, $49.2054^{\circ} \mathrm{N}, 19.69722^{\circ} \mathrm{E}$, alt. $1770 \mathrm{~m}, 1810$ m, 1815 m, 1895 m, leg. PG, 17.07.2008, 20.07.2008 (POZNB 1/2008, 2/2008, 3/2008); 34UDV0650: Spálená dolina valley, below Baníkovské sedlo pass, alt. 1830 m, leg. PG, 19.07.2006 (POZNB 39); Spálená dolina valley, below rocky walls descending from $\mathrm{Mt}$ Baníkov, alt. 1875 m, 1885 m, leg. PG, 19.07.2006 (POZNB 3/2006, 5/2006); 34UDV0751: Roháčska dolina valley, blocks of rock near Horne Roháčske pleso lake, alt. $1730 \mathrm{~m}, 1755 \mathrm{~m}$, leg. PG, 24.07.2006 (POZNB 21/2006, 22/2006); Spálená dolina valley, rocky outcrops near route, above Rázcestie k Roháčskym Plesám, alt. 1530 m, leg. PG, 24.07.2006 (POZNB 20/2006); 34UDV0849: Žiarska dolina valley, Malé Závraty, below Žiarskie sedlo pass, in the glacial cirque below Mt Plačlivý Roháč, alt. 1785 m, leg. PG, 20.07.2006 (POZNB 9/2006); Žiarska dolina valley, Malé Závraty, glacial cirque between Žiarskie sedlo pass and Mt Smrek, alt. 1790 m, leg. PG, 20.07.2006 (POZNB 10/2006); 34UDV0949: snowbed on a ridge between Žiarskie sedlo pass and Mt Smrek, alt. 2025 m, leg. PG, 21.07.2006 (POZNB 13/2006, 14/2006); 34UDV0950: Mt Ostrý Roháč, rock crevices on northern slope, alt. $1995 \mathrm{~m}$, leg. PG, 26.08.2008 (POZNB 733); Smutná dolina valley, below rocky walls descending from Mt Ostrý Roháč, alt. $1810 \mathrm{~m}$, leg. PG, 18.07.2006 (POZNB 1/2006); Zadná Jamnícka dolina valley (Kocioł Jamnickich Stawów), near $\mathrm{W}$ shore of Jamnícke Vyšné pleso lake, alt. $1720 \mathrm{~m}, 1830 \mathrm{~m}$, leg. PG, 25.08.2008 (POZNB 35/2008, 36/2008); 34UDV1050: Zadná Jamnícka dolina valley (Kocioł Jamnickich Stawów), above Jamnícke Nižne pleso lake, alt. 1760 m, leg. PG, 25.08.2008 (POZNB 30); 34UDV1250: Račkova dolina valley, Račkov Zadok, blocks of rock near Vyšné Račkove pleso lake, alt. 1715 m, leg. PG, 21.07.2009 (POZNB 3/2009); Račkova dolina valley, Račkov Zadok, glacial cirque below Mt Jakubiná, alt. $1810 \mathrm{~m}$, 1830 m, 1845 m, leg. PG, 10.08.2004, 21.07.2009 (POZNB 4/2004, 5/2004, 1/2009, 2/2009); Spálená dolina valley, below Baníkovské sedlo pass, rocky outcrops E from a route, alt. $1875 \mathrm{~m}$, leg. PG, 19.07.2006 (POZNB 2/2006); 34UDV1548: Kobyla ridge (SE from Mt Bystrá), rocky outcrops near route to Bystrá dolina valley, Junco-Oreochloetum salicetosum herbaceae, $49.18053^{\circ} \mathrm{N}, 19.84937^{\circ} \mathrm{E}, 49.18702^{\circ} \mathrm{N}, 19.84545^{\circ} \mathrm{E}$, alt. $1955 \mathrm{~m}, 2175 \mathrm{~m}$, leg. PG, 26.08.2011 (POZNB 452, 618); 34UDV1649: upper part of Kamenistá dolina valley, glacial cirque below Mt Blyšt, alt. 1845 
m, leg. PG, 29.08.2008 (POZNB 39/2008); upper part of Kamenistá dolina valley, glacial cirque below Mt Blyšt, close to Pyšne sedlo pass, alt. $1865 \mathrm{~m}, 1875 \mathrm{~m}$, leg. PG, 29.08.2008 (POZNB 37/2008, 38/2008).

Poland, High Tatra Mountains: 34UDV2752: Dolina Gąsienicowa valley, glacial cirque below Mt Świnica, above Czerwone Stawki lakes, Grzędy, below rocky walls descending from Mt Skrajna Turnia, alt. 1805 m, $1870 \mathrm{~m}, 1875 \mathrm{~m}$, leg. PG, 20.08.2005 (POZNB 18/2005, 19/2005, 20/2005); Dolina Gąsienicowa valley, glacial cirque below Mt Świnica, rocky outcrops S from Zadni Staw Gąsienicowy lake, alt. 1910 m, leg. PG, 20.08.2005 (POZNB 24/2005); 34UDV2753: Dolina Gąsienicowa valley, near Kurtkowiec lake, $49.22845^{\circ} \mathrm{N}$, $20.00313^{\circ} \mathrm{E}$, alt. $1685 \mathrm{~m}$, leg. PG, 30.06.2010 (POZNB 195); Dolina Gąsienicowa valley, near $S$ shore of Długi Staw lake, Salicetum herbaceae, $49.22595^{\circ} \mathrm{N}$, 20.00882 ${ }^{\circ}$, alt. $1780 \mathrm{~m}$, leg. PG, 20.09.2010 (POZNB 276); 34UDV2850: Dolina Pięciu Stawów Polskich valley, below Kotelnica ridge, above Czarny Staw Polski lake, alt. $1835 \mathrm{~m}, 1845 \mathrm{~m}$, leg. PG, 27.07.2005 (POZNB 15/2005, 16/2005); 34UDV2851: W part of Dolina Pięciu Stawów Polskich valley, snowbeds below Gładka Przełęcz pass, alt. 1850 m, leg. PG, 27.07.2005 (POZNB 17/2005); Dolina Pięciu Stawów Polskich valley, sub-glacially eroded step of Dolinka Pusta valley, blocks of rock, $49.21427^{\circ} \mathrm{N}, 20.0227^{\circ} \mathrm{E}$, alt. $1960 \mathrm{~m}$, leg. PG, 24.08.2012 (POZNB 1964); 34UDV2852: Dolina Gąsienicowa valley, above and SW from Zmarzły Staw Gąsienicowy lake, alt. 1800 m, leg. PG, 1.08.2003 (POZNB 101/2003); Dolina Gąsienicowa valley, above Zmarzły Staw Gąsienicowy lake, blocks of rock, alt. $1800 \mathrm{~m}, 1815 \mathrm{~m}, 1845 \mathrm{~m}$, leg. PG, 2.08.2003, 21.08.2005 (POZNB 286, 931, 29/2005, 30/2005); Dolina Gąsienicowa valley, at the mouth of Zawratowy Żleb gully, alt. 1955 m, leg. PG, 1.08.2003 (POZNB 106/2003); Dolina Gąsienicowa valley, below rocky walls descending from Mt Zmarzła Przełęcz pass, alt. 2015 m, leg. PG, 4.08.2003 (POZNB 120/2003, 121/2003); Dolina Gąsienicowa valley, below rocky walls descending from Mt Zmarzłe Czuby, alt. $1930 \mathrm{~m}$, leg. PG, 4.08.2003 (POZNB 117/2003, 118/2003, 119/2003); Dolina Gąsienicowa valley, below Zawratowy Żleb gully, alt. $1825 \mathrm{~m}, 1840 \mathrm{~m}$, leg. PG, 1.08.2003 (POZNB 103/2003, 104/2003, 107/2003); Dolina Gąsienicowa valley, on the sub-glacially eroded step of Dolinka Kozia valley, alt. 1810 m, leg. PG, 21.08.2005 (POZNB 26/2005); Dolina Gąsienicowa valley, $\mathrm{S}$ and above Zmarzły Staw Gąsienicowy lake, alt. $1800 \mathrm{~m}$, leg. PG, 1.08.2003, 2.08.2003 (POZNB 98/2003, 100/2003, 112/2003); Dolina Gąsienicowa valley, Zadnie Koło, glacial cirque below Mylna Przełęcz pass, $49.22275^{\circ} \mathrm{N}, 20.01258^{\circ} \mathrm{E}$, alt. $1975 \mathrm{~m}$, leg. PG, 30.08.2011 (POZNB 680); 34UDV2853: Dolina Gąsienicowa valley, above NW shore of Czarny Staw Gąsienicowy lake, alt. 1680 m, leg. PG, 31.07.2003 (POZNB 95/2003); Dolina Gąsienicowa valley, above W shore of Czarny Staw Gąsienicowy lake, at the mouth of Żleb Zaruskiego gully, alt. 1720 m, leg. PG, 31.07.2003 (POZNB 96/2003); Dolina Gąsienicowa valley, below Zmarzły Staw lake, slighty below where the paths to Dolinka Kozia valley and Zawrat pass cross, alt. $1750 \mathrm{~m}$, leg. PG, 31.07.2003 (POZNB 89/2003); Dolina Gąsienicowa valley, glacial cirque below Mt Świnica, blocks of rock near Długi Staw Gąsienicowy lake, alt. 1845 m, leg. PG, 20.08.2005 (POZNB 21/2005); Dolina Gąsienicowa valley, near E shore of Czarny Staw Gąsienicowy lake, near route to Granaty ridge, alt. $1640 \mathrm{~m}$, leg. PG, 31.07.2003 (POZNB 90/2003, 91/2003); Dolina Gąsienicowa valley, rocky rubble above Czarny Staw Gąsienicowy lake, below Mt Kościelec, alt. 1630 m, leg. PG, 31.07.2003 (POZNB 932); Dolina Gąsienicowa valley, blocks of rock above W shore of Czarny Staw Gąsienicowy lake, alt. $1650 \mathrm{~m}$, leg. PG, 31.07.2003 (POZNB 92/2003, 93/2003, 94/2003); Dolina Gąsienicowa valley, above S shore of Czarny Staw Gąsienicowy lake, below rocky walls descending from Mt Kościelec, alt. 1720 m, leg. PG, 31.07.2003 (POZNB 97/2003); 34UDV2950: Dolina Pięciu Stawów Polskich valley, below Czarna Ławka pass, alt. $1870 \mathrm{~m}$, leg. PG, 27.07.2005 (POZNB 12/2005, 13/2005, 14/2005); Dolina Pięciu Stawów Polskich valley, below rocky walls descending from Mt Wyżni Liptowski Kostur, alt. 1940 m, 2040 m, leg. PG, 25.07.2005 (POZNB 1/2005, 2/2005); 34UDV2952: Dolinka Kozia valley, rocky rubble filling the valley bottom, $49.222^{\circ} \mathrm{N}, 20.02657^{\circ} \mathrm{E}$, alt. $1920 \mathrm{~m}, 1935$ m, 1950 m, 1960 m, leg. PG, 2.08.2003, 20.08.2005, 21.08.2005, 31.08.2011 (POZNB 468, 111/2003, 23/2005, 27/2005, 28/2005); upper part of Dolinka Kozia valley, below rocky walls descending from Orla Perć ridge, alt. 2050 m, 2055 m, leg. PG, 2.08.2003 (POZNB 108/2003, 109/2003); 34UDV2953: Dolina Pańszczycy valley, below rocky walls descending from Pańszczycka Przełęcz pass, alt. 1985 m, 1995 m, leg. PG, 3.08.2003 (POZNB 113/2003, 114/2003, 115/2003); 34UDV3049: Dolina za Mnichem valley, below rocky walls descending from Wrota Chałubińskiego pass, alt. 1850 m, leg. PG, 16.08.2004 (POZNB 17/2004, 18/2004); Dolina za Mnichem valley, above Wyżni Staw Staszica lake, alt. 1820 m, leg. PG, 16.08.2004 (POZNB 16/2004); Dolina za Mnichem valley, below Mt Cubryna, snowbed among blocks of rock, alt. 1900 m, 2000 m, leg. PG, 16.08.2004 (POZNB 19/2004, 20/2004, 22/2004); Dolina za Mnichem valley, below rocky walls descending from Mt Zadni Mnich, alt. 1980 m, leg. PG, 17.08.2004 (POZNB 23/2004); Dolina za Mnichem valley, near Wyżni Staw Staszica lake, alt. 1780 m, leg. PG, 5.09.2004 (POZNB 26); Dolina za Mnichem valley, rocky rubble filling the valley bottom, alt. $1820 \mathrm{~m}$, leg. PG, 16.08.2004 (POZNB 15/2004); 34UDV3050: Dolina Pięciu Stawów Polskich valley, Brzuchaty Piarg scree, below rocky walls descending from Mt Miedziane, alt. 1905 m, leg. PG, 25.07.2005 (POZNB 5/2005); Dolina Pięciu Stawów Polskich valley, Czerwony Piarg scree, near route 
to Szpiglasowa Przełęcz pass, alt. $1955 \mathrm{~m}$, leg. PG, 25.07.2005 (POZNB 4/2005); 34UDV3051: Dolina Pięciu Stawów Polskich valley, S from Przedni Staw Polski lake, alt. 1670 m, leg. PG, 25.07.2005 (POZNB 6/2005); 34UDV3052: Dolina Pięciu Stawów Polskich valley, Buczynowa Dolinka valley, alt. $1795 \mathrm{~m}$, leg. PG, 22.09.2005 (POZNB 34/2005); 34UDV3053: Dolina Waksmundzka valley, below rocky walls descending from Mt Wołoszyn, alt. $1995 \mathrm{~m}$, leg. PG, 22.08.2005 (POZNB 31/2005); 34UDV3148: below Mięguszowiecka Przełęcz pod Chłopkiem pass, rock crevices, $49.18395^{\circ} \mathrm{N}, 20.06592^{\circ} \mathrm{E}, 49.18363^{\circ} \mathrm{N}$, $20.06533^{\circ} \mathrm{E}$, alt. $2305 \mathrm{~m}$, leg. PG, 2.09.2014 (POZNB 1884, 1910); 34UDV3149: Dolina za Mnichem valley, peat-bog near Wyżnie Stawki Staszica lakes, alt. 1855 m, leg. PG, 28.08.2012 (POZNB 1497); Dolina za Mnichem valley, rocky outcrops above Wyżnie Stawki Staszica lakes, alt. 1825 m, 1835 m, leg. PG, 20.09.2005 (POZNB 32/2005, 33/2005); 34UDV3151: Dolina Pięciu Stawów Polskich valley, below rocky walls descending from Marchwiczna Przełęcz pass, alt. 1810 m, 1815 m, 1900 m, 1950 m, leg. PG, 26.07.2005 (POZNB 8/2005, 9/2005, 10/2005, 11/2005); Dolina Pięciu Stawów Polskich valley, Dolinka za Niedźwiedziem valley, alt. $1785 \mathrm{~m}$, leg. PG, 26.07.2005 (POZNB 7/2005); 34UDV3248: below Mięguszowiecka Przełęcz pod Chłopkiem pass, rock crevices, $49.18407^{\circ} \mathrm{N}, 20.06698^{\circ} \mathrm{E}$, alt. $2255 \mathrm{~m}$, leg. PG, 2.09.2014 (POZNB 1904); Dolina Rybiego Potoku valley, rocky outcrops above Czarny Staw pod Rysami lake, alt. 1715 m, leg. PG, 18.08.2004 (POZNB 24/2004).

Poland, Western Tatra Mountains: 34UDV1150: Dolina Jarząbcza valley, below rocky walls descending from Niska Przełęcz pass, alt. 1700 m, 1730 $\mathrm{m}, 1750 \mathrm{~m}$, leg. PG, 9.08.2004 (POZNB 1/2004, 2/2004, 3/2004); 34UDV1251: Dolina Jarząbcza valley, NW slope of Mt Kończysty Wierch, alt. $1650 \mathrm{~m}, 1700 \mathrm{~m}, 1720 \mathrm{~m}, 1735 \mathrm{~m}, 1755 \mathrm{~m}$, leg. PG, 28.09.2003, 10.08.2004, 22.07.2009 (POZNB 560, 8/2004, 4/2009, 5/2009, 6/2009, 7/2009, 8/2009); 34UDV1350: Dolina Starorobociańska valley, Gładka Ubocz (between Mt Starorobociański Wierch and Starorobociańska Przełęcz pass), alt. 1800 m, leg. PG, 12.07.2003 (POZNB 79/2003); 34UDV1351: Dolina Jarząbcza valley, in a gully descending from Mt Kończysty Wierch, alt. 1750 m, leg. PG, 10.08.2004 (POZNB 7/2004); 34UDV1450: Dolina Starorobociańska valley, Szeroki Zagon, alt. $1700 \mathrm{~m}, 1760 \mathrm{~m}$, leg. PG, 12.07.2003 (POZNB 83/2003, 84/2003); Dolina Starorobociańska valley, Zadnie Zagony, alt. 1700 m, leg. PG, 11.08.2004 (POZNB 9/2004, 12/2004, 13/2004); Dolina Starorobociańska valley, Zadnie Zagony, Krzywy Żleb gully, alt. 1655 m, 1700 m, leg. PG, 12.07.2003, 23.07.2009 (POZNB 85/2003, 9/2009); Dolina Starorobociańska valley, Zadnie Zagony, Skrajniak, alt. 1690 m, 1700 m, leg. PG, 12.07.2003 (POZNB 80/2003, 81/2003);
34UDV1749: NW slope of Mt Kamienista, rocky crevices below the summit, $49.19628^{\circ} \mathrm{N}, 19.86753^{\circ} \mathrm{E}$, alt. $2045 \mathrm{~m}$, leg. PG, 4.08.2012 (POZNB 1147); 34UDV2553: Mt Kasprowy Wierch, NW slope below the summit, alt. $1970 \mathrm{~m}$, leg. PG, 30.06.2010 (POZNB 220).

Schistochilopsis opacifolia (Culm. ex Meyl.) Konst. [= Lophozia incisa (Schrad.) Dumort. subsp. opacifolia (Culm. ex Meyl.) R.M. Schust. et Damsh.]

Slovakia, High Tatra Mountains: 34UDV2448: upper part of Kôprovnica valley, rocks below Mt Krížne, $49.18565^{\circ} \mathrm{N}, 19.95745^{\circ} \mathrm{E}, 49.18547^{\circ} \mathrm{N}, 19.95777^{\circ} \mathrm{E}$, alt. $1725 \mathrm{~m}, 1735 \mathrm{~m}$, leg. PG, 29.08.2014 (POZNB 1833, 1854); 34UDV3046: Mlynická dolina valley, below rocky walls descending from Mt Štrbský štít, $49.16622^{\circ} \mathrm{N}, 20.0425^{\circ} \mathrm{E}$, alt. $1975 \mathrm{~m}$, leg. $\mathrm{PG}$, 12.08.2011 (POZNB 655); 34UDV3148: Hincova dolina valley, rocky outcrops near NW shore of Velké Hincovo pleso lake, alt. 1940 m, leg. PG, 12.08.2008 (POZNB 28/2008); 34UDV3445: Zlomisková dolina valley, Kotlina Ladového plesa basin, near SE shore of Ladové pleso lake, $49.16285^{\circ} \mathrm{N}, 20.10755^{\circ} \mathrm{E}$, alt. $1930 \mathrm{~m}$, leg. PG, 6.08.2010 (POZNB 221); Zlomisková dolina valley, on sub-glacially eroded step of upper glacial cirque (Kotlina Ladového plesa basin), near stream, $49.1624^{\circ} \mathrm{N}, 20.1029^{\circ} \mathrm{E}$, alt. 1880 $\mathrm{m}$, leg. PG, 9.08.2011 (POZNB 647); 34UDV3448: upper part of Žabia Bielovodská dolina valley, below rocky walls between Mlynárovo sedlo pass and Mt Velký Žabi štít, alt. 1885 m, leg. PG, 19.08.2009 (POZNB 52/2009, 53/2009); 34UDV3449: Žabia Bielovodská dolina valley, E from S shore of Nižné Žabie Bielovodské pleso lake, below rocky walls descending from Mt Prostredný Mlynár, alt. 1735 m, leg. PG, 16.08.2009 (POZNB 41/2009); 34UDV3546: Kačacia dolina valley, upper (SE) part of lower glacial cirque, alt. $1750 \mathrm{~m}$, leg. PG, 16.09.2009 (POZNB 76/2009); 34UDV3547: Kačacia dolina valley, lower glacial cirque, rock of blocks, alt. $1645 \mathrm{~m}$, 1700 m, leg. PG, 22.08.2007 (POZNB 7/2007, 31); 34UDV3548: Ťažká dolina valley, upper part of the lower glacial cirque, blocks of rock below Mt Pustá stráž, alt. $1810 \mathrm{~m}$, leg. PG, 26.08.2009 (POZNB 68/2009); 34UDV3647: Litvorová dolina valley, below rocky walls descending from Mt Litvorový štít, alt. 1835 m, leg. PG, 15.09.2009 (POZNB 72/2009); Svištová (Bielovodská) dolina valley, Vyšna Svištová roveň, $49.18063^{\circ} \mathrm{N}, 20.13553^{\circ} \mathrm{E}$, alt. $1985 \mathrm{~m}$, leg. PG, 26.09.2011 (POZNB 473); 34UDV4251: Červena dolina valley, near Červené pleso lake, alt. $1815 \mathrm{~m}$, leg. PG, 25.09.2007 (POZNB 32/2007); 34UDV4252: Dolina Bielych plies valley, alt. 1840 m, leg. PG, 26.09.2007 (POZNB 36/2007).

Poland, High Tatra Mountains: 34UDV2752: Dolina Gąsienicowa valley, glacial cirque below $\mathrm{Mt}$ Świnica, above Czerwone Stawki lakes, Grzędy, be- 
low rocky walls descending from Mt Skrajna Turnia, alt. 1870 m, leg. PG, 20.08.2005 (POZNB 19/2005); 34UDV2753: Dolina Gąsienicowa valley, near Czerwone Stawki lakes, $49.22743^{\circ} \mathrm{N}, 20.00405^{\circ} \mathrm{E}$, alt. $1680 \mathrm{~m}$, leg. PG, 15.08.2013 (POZNB 1956); 34UDV2852: Dolina Gąsienicowa valley, above Zmarzły Staw lake, alt. 1800 m, leg. PG, 4.08.2004 (POZNB 981); 34UDV2950: Dolina Pięciu Stawów Polskich valley, below rocky walls descending from Mt Wyżni Liptowski Kostur, alt. 1940 m, leg. PG, 25.07.2005 (POZNB 1/2005); 34UDV3049: Dolina za Mnichem valley, below rocky walls descending from Wrota Chałubińskiego pass, alt. $1850 \mathrm{~m}$, leg. PG, 16.08.2004 (POZNB 18/2004).

Poland, Western Tatra Mountains: 34UDV1150: Dolina Wyżnia Jarząbcza valley, below rocky walls descending from Niska Przełęcz pass, alt. 1700 m, 1715 m, 1750 m, leg. PG, 9.08.2004, 28.09.2006 (POZNB 61, 1/2004, 2/2004); 34UDV1251: Dolina Jarząbcza valley, NW slope of Mt Kończysty Wierch (Jarząbczy Kopieniec), alt. 1720 m, 1735 m, leg. PG, 22.07.2009 (POZNB 6/2009, 7/2009, 8/2009); 34UDV1450: Dolina Starorobociańska valley, Zadnie Zagony, alt. 1700 m, leg. PG, 11.08.2004 (POZNB 12/2004, 13/2004); Dolina Starorobociańska valley, Szeroki Zagon, alt. $1760 \mathrm{~m}$, leg. PG, 12.07.2003 (POZNB 83/2003).

Trilophozia quinquedentata (Huds.) Bakalin $[=$ Tritomaria quinquedentata (Huds.) Buch.]

Belianske TAtra Mountains: 34UDV4056: Havrania dolina valley, Havrania rovien, rocky outcrops, $49.25632^{\circ} \mathrm{N}, 20.18835^{\circ} \mathrm{E}$, alt. $1460 \mathrm{~m}$, leg. PG, 26.06.2012 (POZNB 1281); 34UDV4056: N slope of Mt Malý vrch, shaded calcareous rocks in spruce forest, $49.26665^{\circ} \mathrm{N}, 20.18303^{\circ} \mathrm{E}$, alt. $1205 \mathrm{~m}$, leg. PG, 26.06.2012 (POZNB 1189); 34UDV4156: above SE part of Stará polana glade, gully descending from Mt Nižny Havran, calcareous outcrops, $49.25772^{\circ} \mathrm{N}$, $20.19892^{\circ} \mathrm{E}, 49.25778^{\circ} \mathrm{N}, 20.19847^{\circ} \mathrm{E}$, alt. $1350 \mathrm{~m}$, 1380 m, leg. PG, 24.06.2012 (POZNB 1241, 1290); 34UDV4157: valley of the CCierny potok stream, near Čierny potok stream, $49.26722^{\circ} \mathrm{N}, 20.19503^{\circ} \mathrm{E}$, alt. $1050 \mathrm{~m}$, leg. PG, 24.06.2012 (POZNB 1180); 34UDV4256: lower part of Tristarský žlab gully, near Tristarský potok stream, $49.25782^{\circ} \mathrm{N}, 20.21198^{\circ} \mathrm{E}$, $49.2574^{\circ} \mathrm{N}, 20.21245^{\circ} \mathrm{E}$, alt. $1175 \mathrm{~m}, 1210 \mathrm{~m}$, leg. PG, 25.06.2012 (POZNB 1270, 1288); 34UDV4554: Dolina pod Košiare valley, calcareous outcrops in lower part of Velký Košiar, $49.24065^{\circ} \mathrm{N}, 20.24913^{\circ} \mathrm{E}$, alt. $1380 \mathrm{~m}$, leg. PG, 27.06.2012 (POZNB 1285).

Slovakia, High Tatra Mountains: 34UDV2448: Kôprovnica valley, upper part, rocks below Mt Krížne, $49.18565^{\circ} \mathrm{N}, 19.95745^{\circ} \mathrm{E}$, alt. $1725 \mathrm{~m}$, leg. PG, 29.08.2014 (POZNB 1832); 34UDV2746: Nefcerka valley, below Nižné Terianske pleso lake, rocky outcrops below lake, alt. $1940 \mathrm{~m}$, leg. PG, 2.08.2012 (POZNB 1314); 34UDV2846: Nefcerka valley, blocks of rock $\mathrm{S}$ from Vyšné Terianske pleso lake, $49.1658^{\circ} \mathrm{N}, 20.0198^{\circ} \mathrm{E}$, alt. $2125 \mathrm{~m}$, leg. PG (POZNB 1968); 34UDV3745: Sedielko nad Kotlom pass [in the ridge of Mt Kotlový štít (Malý Gerlach) ], $49.16068^{\circ} \mathrm{N}, 20.14022^{\circ} \mathrm{E}$, alt. $2405 \mathrm{~m}$, leg. PG, 7.08.2013 (POZNB 1933); 34UDV3747: upper part of Svištová (Bielovodská) dolina valley, $49.1811^{\circ} \mathrm{N}$, 20.13717 ${ }^{\circ}$, alt. $1975 \mathrm{~m}$, leg. PG, 26.09.2011 (POZNB 975); 34UDV3948: Zadná Javorová dolina valley, glacial cirque below rocky walls between $\mathrm{Mt}$ Ostrý štít and Mt Javorový štít, alt. 2085 m, leg. PG, 16.08.2006 (POZNB 32/2006); 34UDV4251: Cervena dolina valley, blocks of rock near Belasé pleso lake, alt. 1865 m, leg. PG, 25.09.2007 (POZNB 31/2007). SLOVAKIA, Western TATRA Mountains: 34UDV0657: S slope of Mt Osobitá, open calcareous outcrops above Sedlo pod Osobitou pass, $49.2601^{\circ} \mathrm{N}, 19.72122^{\circ} \mathrm{E}$, alt. 1610 m, leg. PG, 1.10.2013 (POZNB 1697).

Poland, High Tatra Mountains: 34UDV2752: Dolina Gąsienicowa valley, above and S from Zadni Staw Gąsienicowy lake, alpine grassland, on stone, $49.22322^{\circ} \mathrm{N}, 20.00898^{\circ} \mathrm{E}$, alt. $1876 \mathrm{~m}$, leg. PG, 21.09.2010 (POZNB 243); 34UDV2852: Dolina Gąsienicowa valley, below Zawrat pass, rocky ledge with Racomitrium lanuginosum, $49.22152^{\circ} \mathrm{N}$, 20.01895 ${ }^{\circ}$, alt. $1910 \mathrm{~m}$, leg. PG, 8.08.2012 (POZNB 1980); 34UDV3049: Dolina za Mnichem valley, below rocky walls descending from Mt Cubryna, alt. 2000 m, leg. PG, 16.08.2004 (POZNB 20/2004); 34UDV3148: Mięguszowiecka Przełęcz pod Chłopkiem pass, rocky crevices, $49.18363^{\circ} \mathrm{N}, 20.06533^{\circ} \mathrm{E}$, alt. 2307 m, leg. PG, 2.09.2014 (POZNB 1884); 34UDV3248: below Mięguszowiecka Przełęcz pod Chłopkiem pass, rocky crevices, $49.18407^{\circ} \mathrm{N}$, 20.06835 $\mathrm{E}$, alt. $2200 \mathrm{~m}$, leg. PG, 2.09.2014 (POZNB 1901); near E shore of Czarny Staw pod Rysami lake, scree near SW shore of the lake, alt. $1600 \mathrm{~m}$, leg. PG, 18.08.2004 (POZNB 1105, 1107).

Poland, Western Tatra Mountains: 34UDV1749: NW slopes of Mt Kamienista, $49.19628^{\circ} \mathrm{N}$, $19.86753^{\circ} \mathrm{E}$, alt. $2045 \mathrm{~m}$, leg. PG, 4.08.2012 (POZNB 1151); 34UDV2456: SW part of Polana Kalatówki glade, rocky outcrops in spruce forest, at the mouth of Kalackie Koryto gully, $49.25698^{\circ} \mathrm{N}, 19.96257^{\circ} \mathrm{E}$, alt. 1265 m, leg. PG, 24.11.2011 (POZNB 879, 880).

\section{REFERENCES}

GóRsKi P., VÁŇA J. (2014): A synopsis of liverworts occurring in the Tatra Mountains (Western Carpathians, Poland and Slovakia): checklist, distribution and new data. Preslia 86(4): 381-485.

For citation: GóRSKI P. (2015): A contribution to the snowbed liverwort flora of the Tatra Mountains (Western Carpathians, Poland and Slovakia). Steciana 19(3): 177-201. doi:10.12657/steciana.019.019 